\title{
Aeroservoelastic Stabilization Techniques for Hypersonic Flight Vehicles
}

Samuel Y. Chan, Peter Y. Cheng, and Dale M. Pitt

McDonnell Aircraft Company

McDonnell Douglas Corporation

St. Louis, Missouri

Thomas T. Myers, David H. Klyde,

Raymond E. Magdaleno, and Duane T. McRuer

Systems Technology, Inc.

Hawthorne, California

Prepared for

Langley Research Center

under Contract NAS1-18763

Scptember 1991
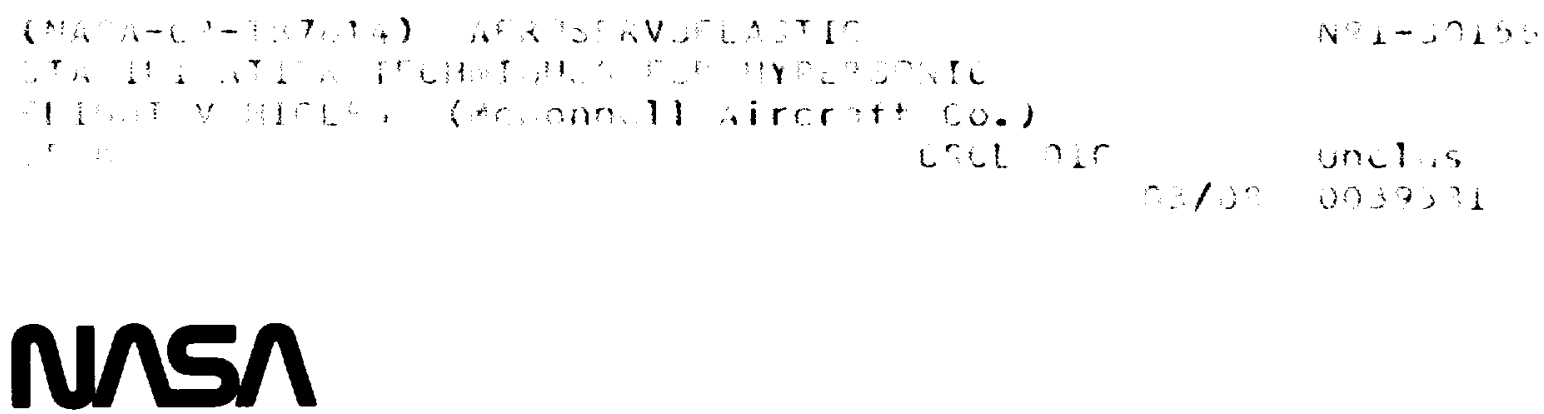

National Aeronautics and

Space Administration

Langley Research Center

Hampton, Virginia 23665-5225 


\section{-}


I. INTRODUCTION ...........................................................................

A. Contract Objective and Scope …………...........................................

B. Overview ..........................................................................................

C. Technical Approach ............................................................................

II. HYPERSONIC FLIGHT VEHICLE MODEL ………….......................... 7

A. Vehicle Configuration ................................................................. 7

B. Vehicle Dynamics ................................................................................... 8

C. Actuator Dynamics ......................................................................... 17

III. FLIGHT CONTROL SYSTEM DESIGN: RIGID BODY LEVEL ........ 19

A. Superaugmented Pitch Loop Concept ........................................... 19

B. Impact of High Frequency Dynamics ............................................... 26

C. Pitch Response Bandwidth Requirement ................................... 30

D. The Baseline System ........................................................................... 32

IV. CONVENTIONAL GAIN STABILIZED DESIGN ............................ 41

A. Ascent Case ................................................................................... 41

B. Descent Case .......................................................................................... 44

V. HYBRID PHASE STABILIZED DESIGN ................................................. 47

A. Ascent Case ….............................................................................. 47

B. Descent Case .................................................................................

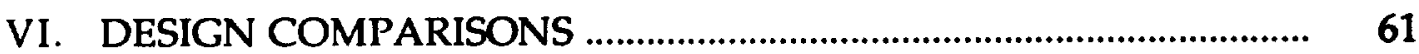

A. Stability Metrics ………..................................................................... 61

B. Residual Response ......................................................................... 63

VII. SUMMARY, CONCLUSIONS AND RECOMMENDATIONS ....... 69

REFERENCES ........................................................................................ 71

APPENDIX A - HSV PROPULSION SYSTEM INTERACTIONS WITH FLEXIBLE MODES AND FLIGHT CONTROL SYSTEMS 


\section{List of Figures}

Page

1. System Survey for Quadratic Dipole Control .............................. 3

2. Hypersonic Flight Vehicle Configuration ................................. $\quad 7$

3. Typical Hypersonic Vehicle Flight Envelope .............................. 8

4. Airframe Modeling …............................................................... 9

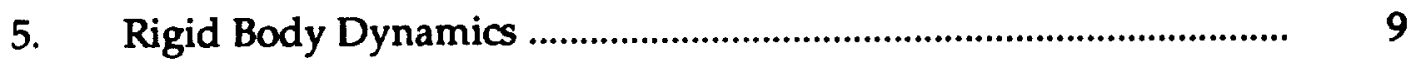

6. Aerodynamic Stability and Control Derivatives ......................... 10

7. Propulsion Effect on Pitch Control Effectiveness ......................... 11

8. Propulsion Effect on Pitch Static Stability .................................... 12

9. NASTRAN BWB-1 Finite Element Model ............................... 12

10. Flexible Vehicle Model Equations ................................................ 13

11. Flexible Pitch Rate Response at FS 84 ......................................... 15

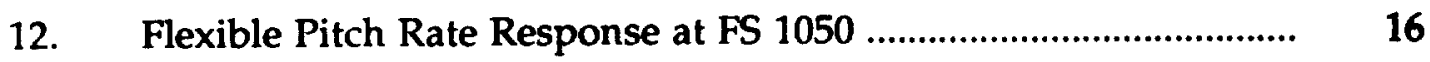

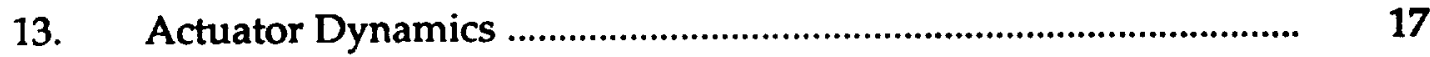

14. Typical Pitch Control System for Superaugmented

Aircraft

15. Superaugmented Pitch Loop as Basis for $\mathrm{n}_{\mathrm{z}}$ or $\alpha$

Command Systems

16. Transfer Function of Short Period Dynamics ............................. 23

17. System Survey Sketch of a Superaugmented Design .................. 24

18. Asymptotic Frequency Response Sketch of the Compensated Open Loop Transfer Function ............................... 27

19. Effect of Time Delay on the Pitch Loop Closure ........................... 29

20. Space Shuttle Pitch Rate Criteria ................................................... 32 


\section{List of Figures (Continued)}

Page

21. Tentative Requirements for Attitude Bandwidth ......................... 33

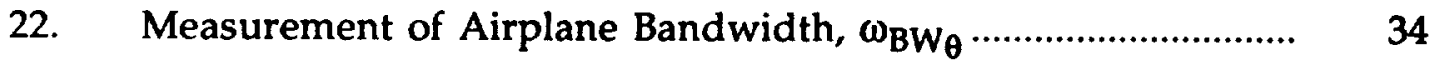

23. Superaugmented Pitch Loop Design to a Specified

Pitch Loop Bandwidth .....

24. Effect of Time Delay on the Rigid Body Pitch Loop

Closure $-\omega_{\mathrm{BW}}=2.0 \mathrm{rad} / \mathrm{sec}$

25. Baseline System Open Loop Frequency Response

- Ascent Case

26. Baseline System Root Locus - Ascent Case

27. Gain Stabilized FCS Open Loop Frequency Response

- Ascent Case .

28. Gain Stabilized FCS Root Locus - Ascent Case

29. Baseline System Open Loop Frequency Response

- Descent Case

30. Gain Stabilized FCS Open Loop Frequency Response

- Descent Case

31. Pitch Loop Closure at the First Bending Mode for Each Sensor Position

32. Sensor Blending to Position First Flexible Mode Zero

33. Positioning First Flexible Mode Zero with Pure Gain Sensor Blend

34. Positioning First Flexible Mode Zero with Filtered Sensor Blend

35. Hybrid Phase Stabilized Design (Blended Sensors

\& First Order Lag

36. Pitch Loop Closure with Sensor Blending (12 rad/sec

Blending Filter) - Ascent Case 


\section{List of Figures (Continued)}

Page

37. Phase Stabilized Design Open Loop Frequency Response (Blended Sensors \& First Order Lag) - Ascent Case

38. Phase Stabilized Design Closure (Blended Sensors \& First Order Lag) - Ascent Case

39. Phase Stabilized FCS Open Loop Frequency Response

- Descent Case

40. Pitch Rate Response to a Unit Step Pitch Rate Command

- Descent Case

41. Response Comparison of Systems, Pitch Rate Response to Unit Pitch Rate Command - Ascent Case

42. Response Comparison of Systems, Elevator

Response to Unit Pitch Rate Command

43. Response Comparison of Systems, Estimated Normal Acceleration Response to Unit Pitch Rate Command

44. Residual Response Metric

\section{List of Tables}

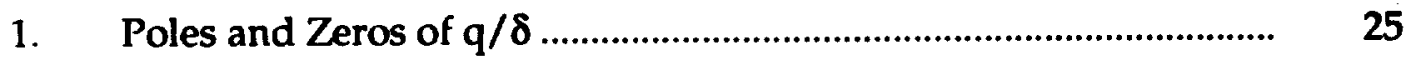

2. Approximate Time Delay Survey of Operational Aircraft ......... $\quad 30$

3. Design of Pitch Loop to Specified Bandwidth .............................. 36

4. Filters Used in Gain Stabilized FCS Designs ............................. 41

5. Stability Metric Comparison - Ascent Case .................................. 61

6. Stability Metric Comparison - Descent Case ............................... $\quad 62$

7. Residual Response Metric Comparison - Ascent Case ................ 67 


\section{Nomenclature and Symbols}

$\mathrm{dB}$

Decibel

c.g.

G

$\mathrm{G}_{\mathrm{c}}$

$\mathrm{G}_{\mathrm{CL}}$

$\mathrm{G}_{\mathbf{f}_{\mathbf{a}}}$

$\mathrm{G}_{\mathrm{f}_{\mathrm{f}}}$

$\mathrm{G}_{\mathrm{LAG}}$

$\mathrm{G}_{\mathbf{M}}$

$\mathrm{G}_{\mathrm{OL}}$

$\mathrm{K}_{\mathbf{a}}$

$\mathrm{K}_{\mathbf{f}}$

$\mathrm{K}_{\mathrm{q}}$

q

$\mathrm{q}_{\mathrm{c}}$

M

$\mathrm{M}_{\alpha}$

$\mathrm{M}_{\delta}$

$\mathrm{n}_{\mathrm{z}}$

$\mathrm{T}_{\mathrm{a}}$

$\mathrm{U}_{\mathrm{O}}$

$\mathrm{Z}_{\delta}$

$\mathrm{Z}_{\mathrm{w}}$

$1 / \mathrm{T}_{\mathrm{sp}_{1}}$

$1 / \mathrm{T}_{\mathrm{sp}_{2}}$

$1 / \mathrm{T}_{\mathrm{h}_{1}}$

$1 / \mathrm{T}_{\theta_{2}}$

$1 / \mathrm{T}_{\mathrm{q}}$

$\alpha$

$\delta$

$\phi_{\mathrm{M}}$

$\tau$

$\tau_{e}$

Center of gravity

Airframe transfer function

Superaugmented pitch loop forward path controller

Superaugmented pitch loop closed loop transfer function

Aft sensor signal blending filter

Forward sensor signal blending filter

High frequency gain stabilized lag filter

Gain margin

Superaugmented pitch loop open loop transfer function

Aft sensor signal blending filter gain

Forward sensor signal blending filter gain

Superaugmented pitch loop equalization gain

Pitch rate

Pitch rate command

Mach

Pitch acceleration per unit angle-of attack

Pitch acceleration per unit control surface deflection

Load factor

First order actuator model time constant

Reference speed

Vertical acceleration per unit control surface deflection

Vertical acceleration per unit vertical velocity

Airframe stable pole

Airframe unstable pole

Zero of altitude to control surface deflection transfer function

Zero of pitch rate to control surface deflection transfer function

Zero of the superaugmented pitch loop equalization transfer function

Angle-of attack

Pitch control surface deflection

Phase margin

Effective time delay

Equivalent system time delay 


\section{Nomenclature and Symbols (Continued)}

$\begin{array}{ll}\tau_{M} & \text { Open loop time delay margin } \\ \omega_{B W_{\theta}} & \text { Pitch attitude bandwidth } \\ \omega_{c} & \text { Zero dB gain crossover frequency } \\ \omega_{n} & \text { Undamped natural frequency } \\ \zeta & \text { Damping ratio }\end{array}$

\section{Acronyms and Abbreviations}

$\begin{array}{ll}\text { ABICS } & \text { Ada Based Integrated Control System } \\ \text { BWB } & \text { Blended Wing Body } \\ \text { DOF } & \text { Degrees of freedom } \\ \text { EOM } & \text { Equations of motion } \\ \text { FCS } & \text { Flight control system } \\ \text { FS } & \text { Fuselage station } \\ \text { HSV } & \text { Hypersonic vehicle } \\ \text { HPS } & \text { Hybrid phase stabilization } \\ \text { IRAD } & \text { Independent Research and Development } \\ \text { MCAIR } & \text { McDonnell Aircraft Company } \\ \text { NASP } & \text { National Aerospace Plane } \\ \text { RCAH } & \text { Pitch rate command, attitude hold } \\ \text { S/MTD } & \text { STOL Maneuver Technology Demonstrator } \\ \text { SSTO } & \text { Single-stage-to-orbit } \\ \text { STI } & \text { Systems Technology, Incorporated }\end{array}$




\section{Section I}

\section{INTRODUCTION}

\section{A. Contract Objective and Scope}

The purpose of this task was to develop aeroservoelastic stabilization techniques for statically unstable hypersonic vehicles (HSVs) and to identify deficiencies in MIL-F-9490D (Reference 1) and MIL-F-87242 (Reference 2) leading to the eventual development of new design requirements for structural mode stabilization of these vehicles. The aeroservoelastic stabilization techniques were developed from generic structural models of HSVs using a suitable control system architecture. The definition of flight and structural conditions for the design, analysis, and evaluation of the developed stabilization techniques was part of this task.

\section{B. Overview}

\section{Flight Control Design Requirements for Flexible Aircraft}

The flight control system (FCS) specifications, MIL-F-9490D and MIL-F-87242, require at least a $\pm 8 \mathrm{~dB}$ gain margin and at least a \pm 60 degree phase margin for frequencies at and above the first structural mode. In practice, this $8 \mathrm{~dB}$ gain margin requirement has often been interpreted by many engineers as attaining an $8 \mathrm{~dB}$ peak clearance below the $0 \mathrm{~dB}$ line for all structural modes. This interpretation of attaining an $8 \mathrm{~dB}$ peak clearance is obviously much more stringent than achieving an $8 \mathrm{~dB}$ gain margin.

Other specifications such as the airplane strength spec. (MIL-A-008870A, Reference 3) and aircraft structures spec. (AFGS-87221A, Reference 4) require a phase margin of at least \pm 60 degrees, but a gain margin of only $\pm 6 \mathrm{~dB}$. In fact, MIL-F-87242 commented that a $\pm 6 \mathrm{~dB}$ gain margin and $\mathrm{a} \pm 45$ degree phase margin are generally agreed to be adequate. A similar statment is also made in AFGS-87221A.

Advanced high performance vehicles, including single-stage-to-orbit (SSTO) hypersonic flight vehicles, that are statically unstable, will require higher bandwidth flight control systems to compensate for the instability resulting in interactions between the flight 
control system, the engine/propulsion dynamics, and the low frequency structural modes. Military specifications, such as those mentioned in previous paragraphs, tend to limit stability margin requirements of structural modes to conventional gain stabilization techniques using notch and low pass filters. The conventional gain stabilization technicyues, however, introduce low frequency effective time delays which can be troublesome from a flying qualities standpoint. These time delays can be alleviated by appropriate blending of gain and phase stabilization techniques for the low frequency structural modes. This possibility is not addressed in the MIL-spec requirements.

\section{The Hybrid Phase Stabilization Concept}

The basic concept of phase stabilization is well established and has been analyzed and applied in the past (e.g., References 5 and 6). The basic principle can be understood from Figure 1. Flexible aircraft responses are characterized by structural mode dipoles that appear at intervals, generally close to the imaginary axis. The stability of the closed loop roots associated with these dipoles, when a FCS is applied, is fundamentally influenced by the ordering of the airframe pole and zero. If the pole appears first (at lower frequency than the zero) as shown in Figure 1a, then the phase curve dips down as it passes through the dipole. This causes the FCS root locus to bulge toward the right half plane creating a propensity for closed loop instability.

Figure $1 \mathrm{~b}$ shows the opposite situation where the zero is below the pole. Here the root locus bulges to the left, fundamentally improving the prospects for stability. The practical design problem then becomes one of creating the favorable dipole constellation. The open loop poles represent unalterable characteristics, since the FCS loop is to be used to position the poles. The airframe zeros can be positioned though, by appropriate positioning of sensors (References 5 and 6). In principle, a sequence of the lower frequency dipoles can be properly arranged (the "saw tooth Bode") such that all of the primary flex modes can be phase stabilized.

Successful application of phase stabilization requires not merely guaranteeing the absence of instability, but also achieving some minimum level of structural mode damping so that uncommanded response due to the structural modes is acceptably low. However, phase stabilization of structural modes may become less feasible for higher frequency modes due to general uncertainty of structural mode characteristics at higher frequencies. 

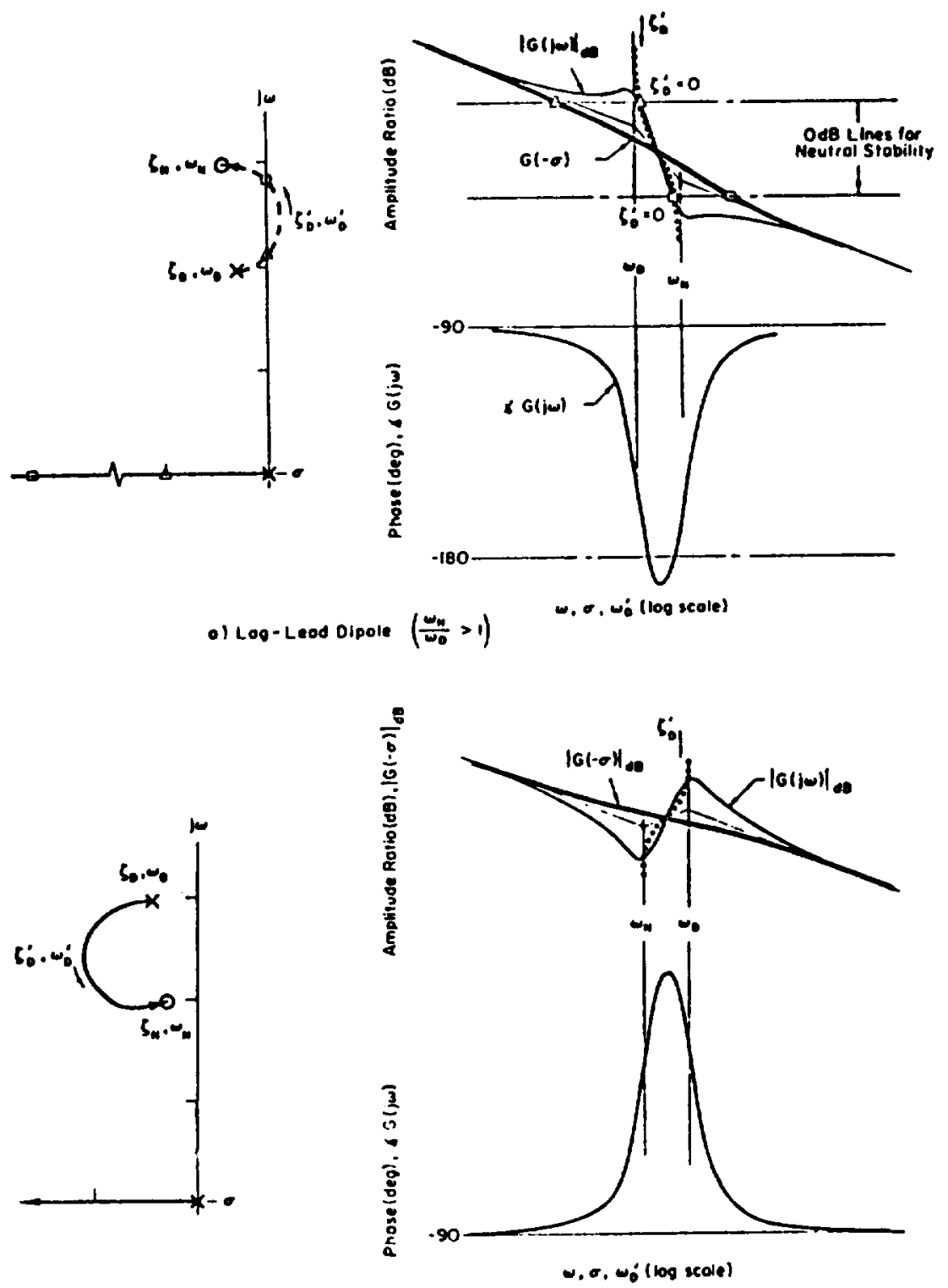

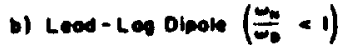

Figure 1. System Survey for Quadratic Dipole Control 
This leads to the concept of Hybrid Phase Stabilization (HPS) investigated in this study, where only a few lower frequency modes would be phase stabilized with the more robust conventional gain stabilization used at higher frequency.

The potential advantage of phase stabilization is in the reduction of the high frequency lags associated with notch and lag filters. As will be seen in the FCS analysis to follow, the stability margins of statically unstable HSVs may be problematic even at the rigid body level. The structural model developed in this study (Section II-C) indicates that the first structural mode may be quite low. The lowest frequency modes are of most concern for conventional designs because they generally must be treated with notch filters. The lower their frequency, the higher the resultant effective time delay penalty.

However, reducing effective time delay is not the only design consideration. The design must also produce acceptably low magnitude high frequency response. In fact, this is what the gain margin specification for structural modes is intended to insure. Phase stabilization is complicated by the fact that its effect on high frequency uncommanded response (referred to as "residual response") is more complex than the effect on time delay. Phase stabilization has the potential for significant increases in structural mode damping ratios. However, what can actually be achieved in practical designs is more difficult to predict (compared to potential time delay reductions) without detailed analysis. Such analyses will be made later, but there is a deeper question of the relevance of the MIL-spec to phase stabilized designs; this will be addressed in Section VI-B.

The details of HPS are best explained in the context of a detailed design example. This will be done in Section V.

\section{Technical Approach}

The existing MIL-spec gain and phase margin requirements were developed primarily with conventional gain stabilization in mind. The relevance of these requirements to other structural stabilization techniques such as phase stabilization is not known. Additional measures such as the residual response metric must be developed to provide guidance in assessing phase stabilization.

A "generic" HSV configuration is presented in Section II. The rigid body dynamics, along with the flexible vehicle model is developed. Note that only the longitudinal 
dynamics are considered here. In this section, the static propulsion effect of the HSV configuration used in this contract is also presented. A comprehensive discussion of the HSV propulsion system interaction with flexible modes and FCS is given in Appendix A. A realistic actuator model is also included in Section II.

The FCS design begins in Section III with the development of a baseline control system architecture -- the superaugmented pitch loop. The fundamentals of the superaugmented pitch loop, and consequently the motivation for selecting this architecture, is explained. The time delay effect on loop closure is addressed. The low and high frequency dynamics peculiar to HSVs are identified and discussed. The pitch loop bandwidth requirement is also discussed in Section-III. This is an important issue since there is a strong correlation between adverse time delay effect and high pitch loop bandwidth. Finally, the baseline FCS, i.e., the superaugmented pitch loop applied to the flexible vehicle but without any structural compensation, is presented in this section. The details of the methods used to design the superaugmented pitch loop at the rigid body level is included in Appendix B.

Beginning in Section IV, designs are generated for two flight conditions: (1) ascent and (2) descent, both at Mach 6. The results of a conventional gain stabilized design using notch and low pass filters are summarized in Section IV. The details of the hybrid phase stabilized design are developed in Section $V$ by means of an example. The comparison of a conventional gain stabilized design to the hybrid phase stabilized design is done in Section VI. First, the stability metrics, which include gain and phase margins and equivalent system time delay, are examined. Then an alternative criterion, the residual response metric, is developed and used to provide additional insights for comparing the two designs.

Section VII follows with a summary and conclusions of the work done in this contract. Recommendations for future work are also included in this section. 
This page is intentionally left blank. 


\section{Section II}

\section{HYPERSONIC FLIGHT VEHICLE MODEL}

\section{A. Vehicle Configuration}

The HSV used in this contract is a National Aerospace Plane (NASP) type configuration. It was a preliminary version of the MCAIR NASP configuration which has been declassified. The configuration is referred to as Blended Wing Body (BWB). This preliminary version of the MCAIR BWB configuration is known as BWB-1 and is shown in Figure 2. The primary pitch control is provided by all moving wings. Four pitch rate gyro sensors are available for feedback and signal blending purposes. Two sensors are located forward of the c.g. and the other two aft of the c.g. The fuselage station (FS) 84 sensor is near the pilot station and the FS 1050 sensor is near the spindle of the all moving wings.

Figure 3 shows a typical HSV flight envelope, which covers a very large set of flight conditions. The space shuttle flight envelope is also shown here for comparison.

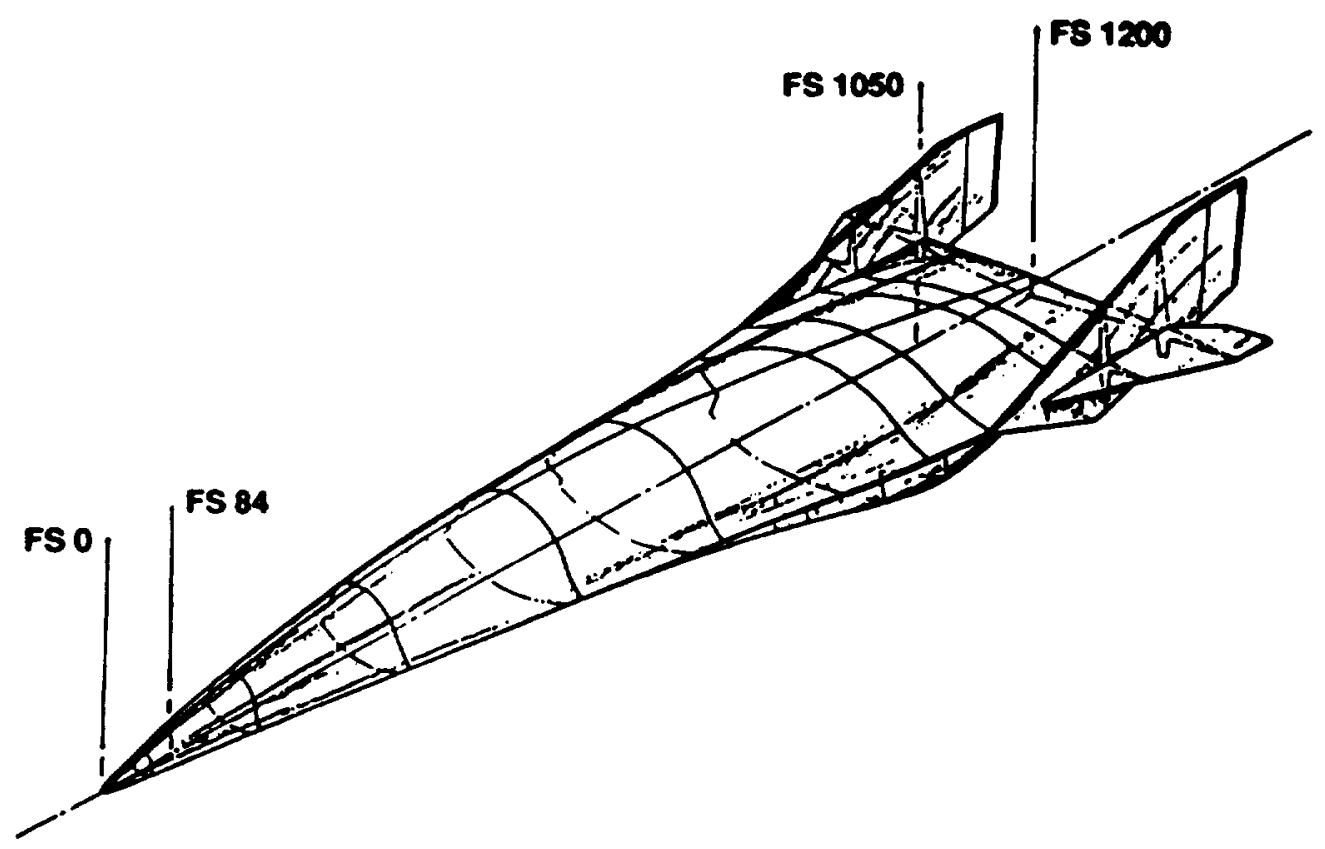

Figure 2. Hypersonic Flight Vehicle Configuration 


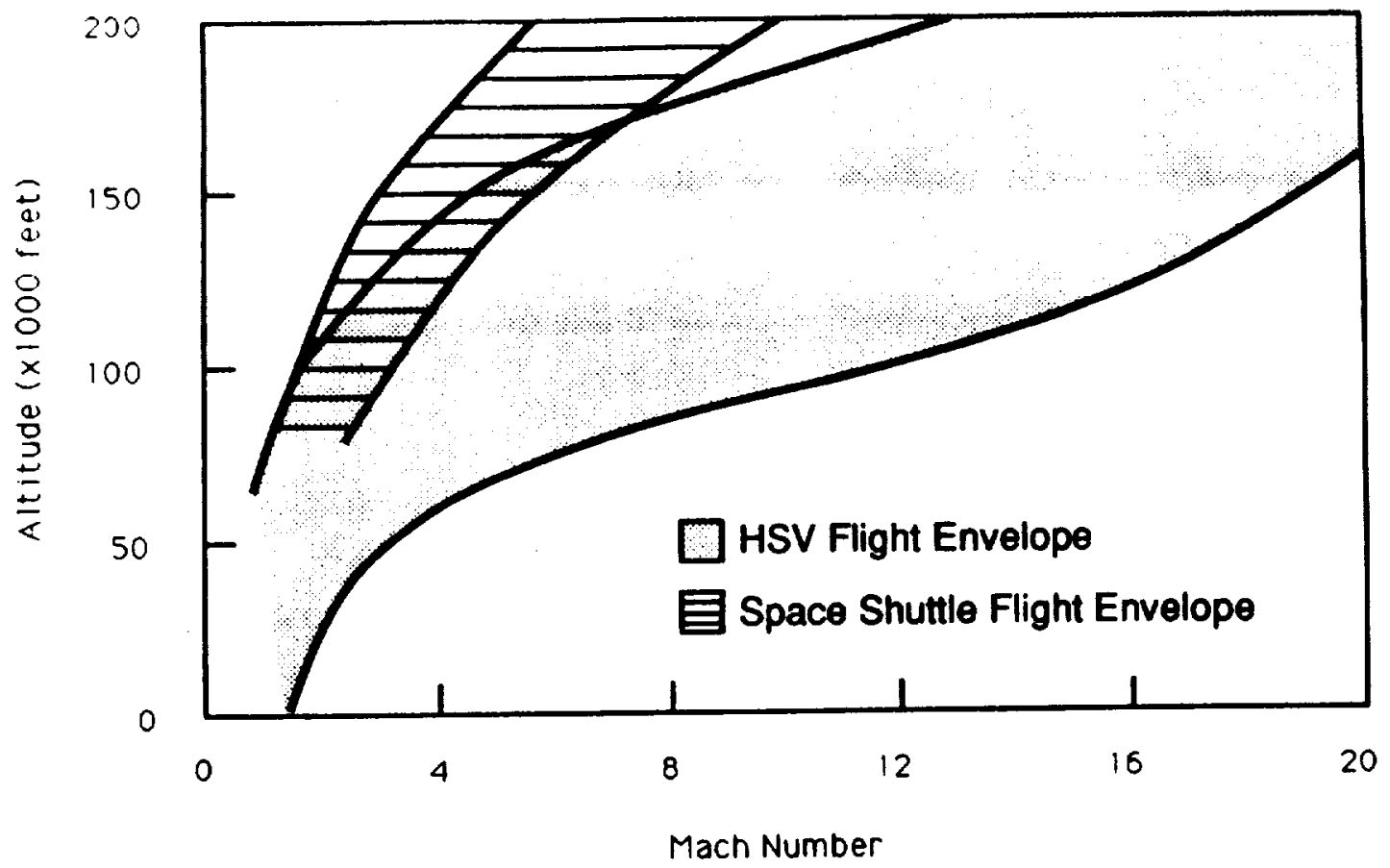

Figure 3. Typical Hypersonic Vehicle Flight Envelope

\section{B. Vehicle Dynamics}

The HSV is modeled with uncoupled rigid body and flexible dynamics as shown in Figure 4 . This is commonly done in fighter aircraft modeling when the system bandwidth is far below the first structural mode frequency. For vehicles having very low structural mode frequencies like the HSVs, it may not be realistic to assume uncoupled rigid body and flexible dynamics, i.e., zero cross-coupling terms in the A-matrix shown in Figure 4. However, for the scope of this contract, the assumption of having zero cross-coupling terms does not invalidate the developed techniques.

\section{Rigid Body Dynamics}

The rigid body longitudinal dynamics are represented by the "short period" approximation as shown in Figure 5. Note that consideration of low frequency dynamics and justification of the short period model are given in Appendix A. Figure 6 shows the aerodynamic stability and control derivatives of the HSV configuration described 
Rigid Body Model

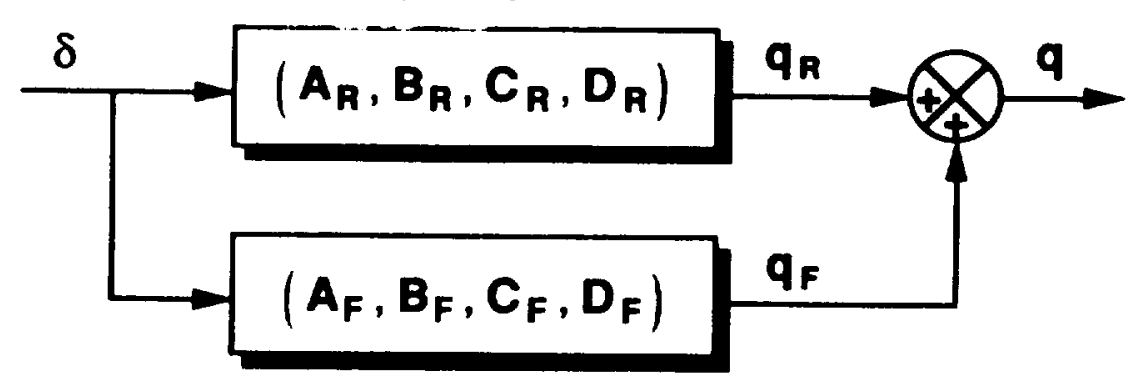

Flexible Model

$$
\left[\begin{array}{c}
\dot{x}_{R} \\
\dot{\bar{x}}_{F}
\end{array}\right]=\underbrace{\left[\begin{array}{cc}
A_{R} & 0 \\
0 & A_{F}
\end{array}\right]}_{A}\left[\begin{array}{l}
\bar{x}_{R} \\
\bar{x}_{F}
\end{array}\right]+\left[\begin{array}{c}
B_{R} \\
B_{F}
\end{array}\right] \delta ; q=\left[C_{R} C_{F}\right]\left[\begin{array}{l}
\bar{x}_{R} \\
\bar{x}_{F}
\end{array}\right]+\left(D_{R}+D_{F}\right) \delta
$$

Figure 4. Airframe Modeling

$$
\begin{aligned}
& {\left[\begin{array}{l}
\dot{\alpha} \\
\dot{q}
\end{array}\right]=\left[\begin{array}{cc}
Z_{\alpha} & 1 \\
M_{\alpha} & M_{q}
\end{array}\right]\left[\begin{array}{l}
\alpha \\
q
\end{array}\right]+\left[\begin{array}{l}
Z_{\delta} \\
M_{\delta}
\end{array}\right] \Delta \delta } \\
& Z_{\alpha}=\frac{\bar{q} S}{m V} C_{Z_{\alpha}} M_{\alpha}=\frac{\bar{q} s \bar{c}}{I_{y y}} C_{m_{\alpha}} \\
& Z_{\delta}=\frac{\bar{q} S}{m V} C_{z_{\delta}} M_{\delta}=\frac{\bar{q} s \bar{c}}{I_{y y}} C_{m_{\delta}} \\
& M_{q}=\frac{\bar{q} s \bar{c}^{2}}{2 I_{y y} V} C_{m_{q}}
\end{aligned}
$$

Figure 5. Rigid Body Dynamics 

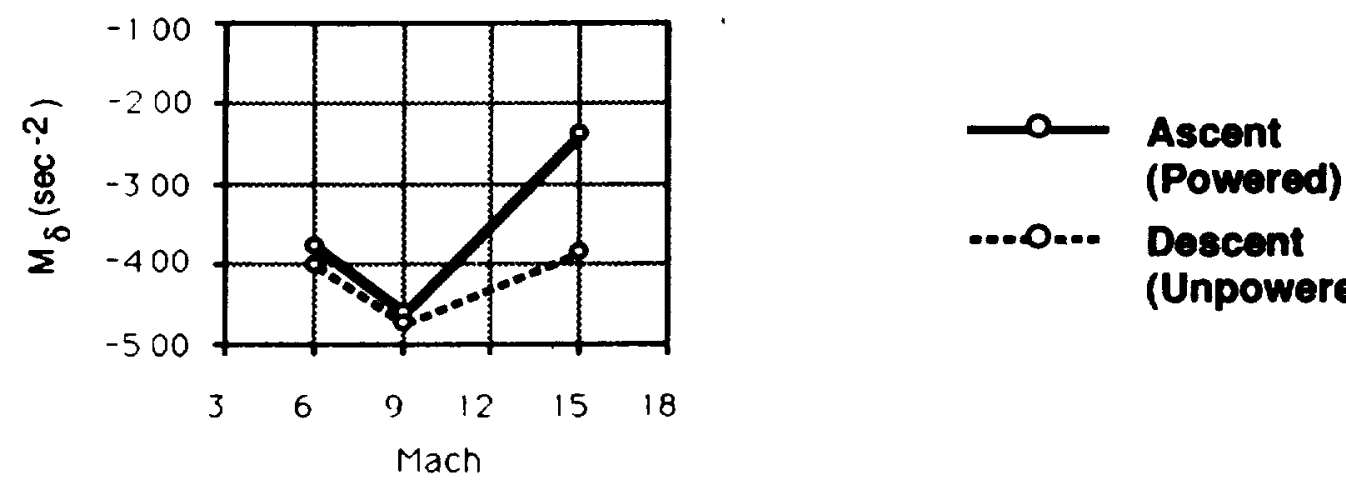

-.0.- Descent

(Unpowered)
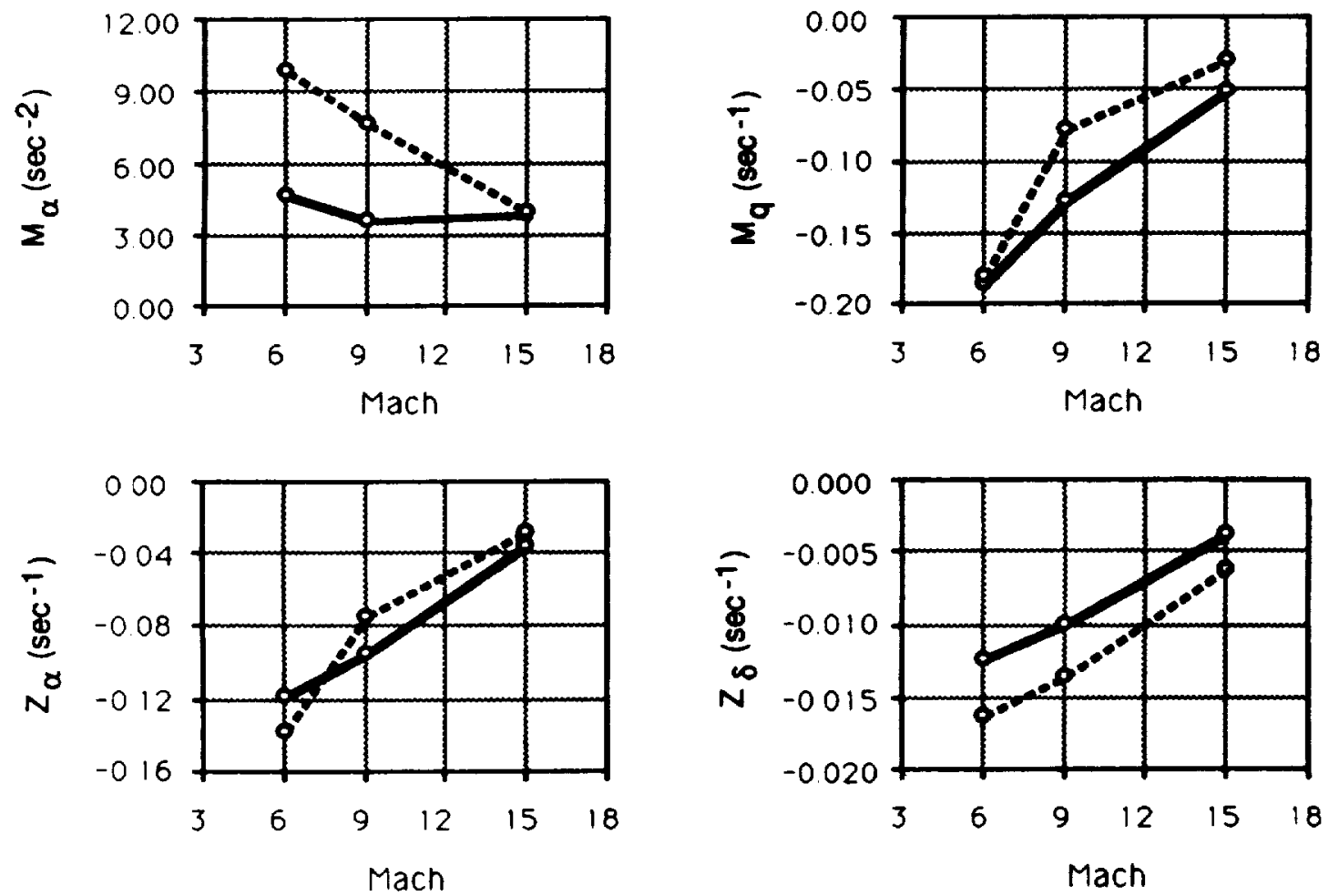

Figure 6. Aerodynamic Stability and Control Derivatives 
previously in Section II-A. The ascent and descent data are typical of flight conditions at high and low dynamic pressure, respectively. These flight conditions encompass a fairly wide range of aircraft static instability.

The propulsion effect is modeled implicitly in the control and stability derivatives. As shown in Figure 7, the propulsion effect on pitch control effectiveness is relatively small. However, significantly larger propulsion effect in pitch control was observed in other HSV configurations. Therefore, it can be concluded that propulsion effect is configuration dependent. Figure 8 shows that, at least for this HSV configuration, the propulsion effect has a greater impact on pitch static stability than pitch control.

\section{Flexible Vehicle Model}

The NASTRAN finite element computer code (Reference 7) was used to generate a finite element model of the BWB-1 structure. The NASTRAN finite element model is shown in Figure 9. All primary and secondary structural members are modeled using QUAD and BAR elements. Non-structural mass such as fuel, avionics, landing gear, etc. are modeled as concentrated mass. A modal analysis was performed using NASTRAN to

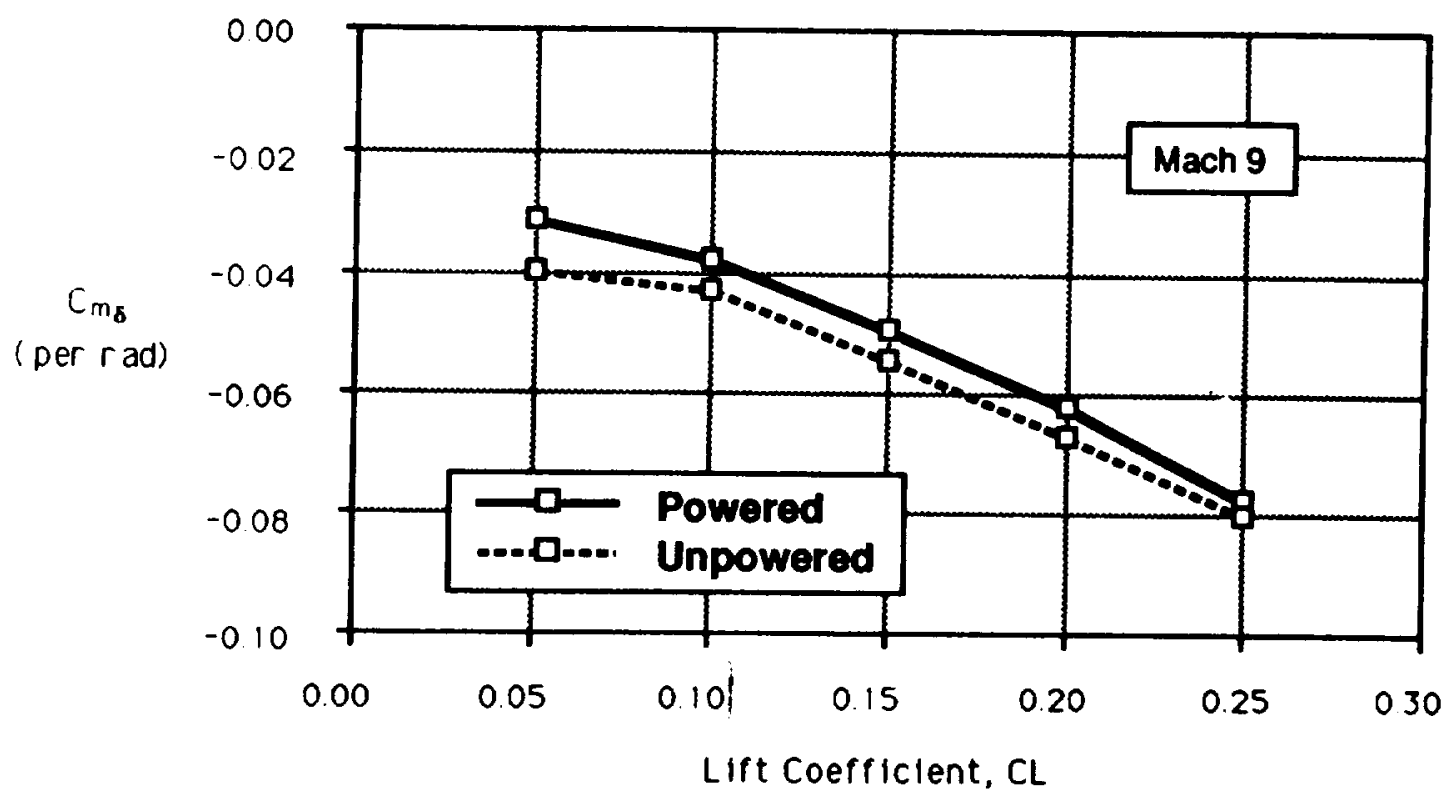

Figure 7. Propulsion Effect on Pitch Control Effectiveness 


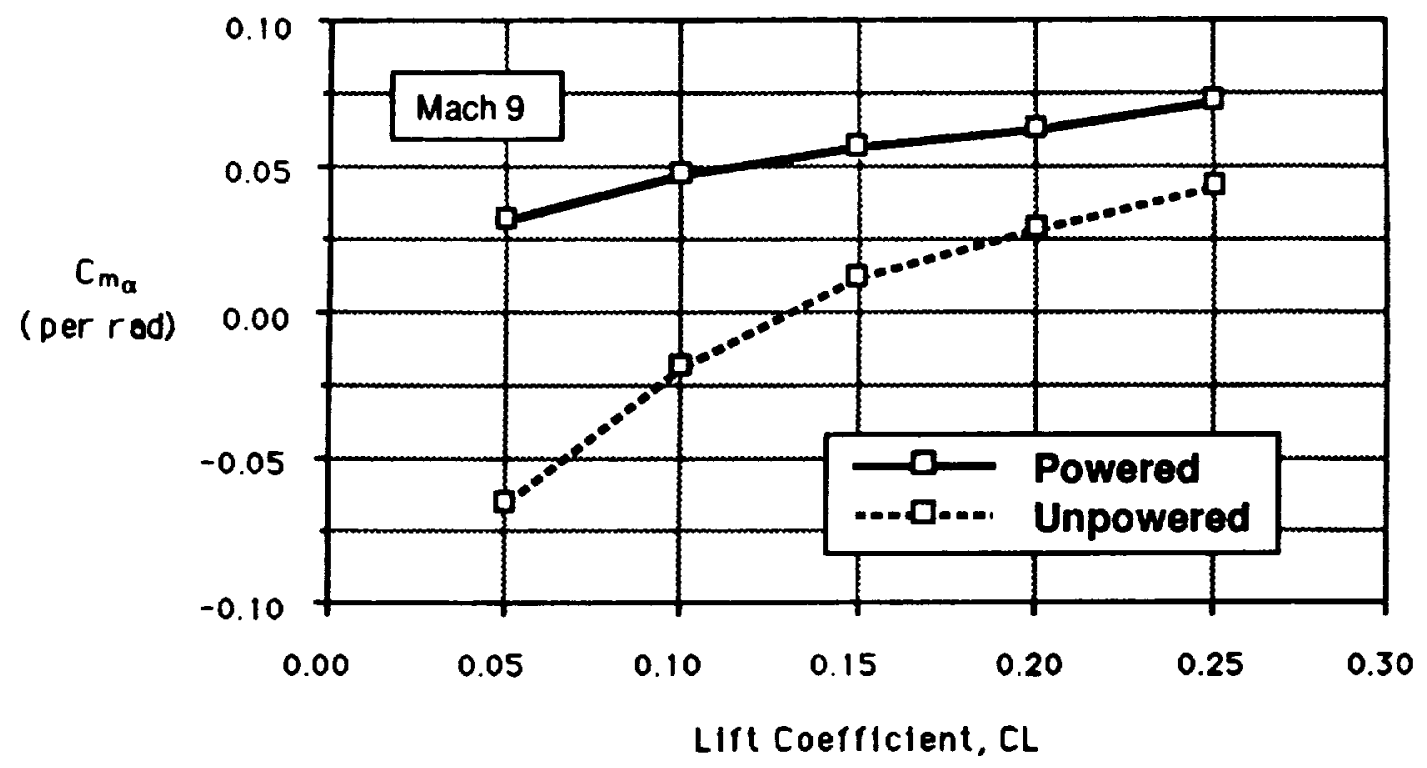

Figure 8. Propulsion Effect on Pitch Static Stability

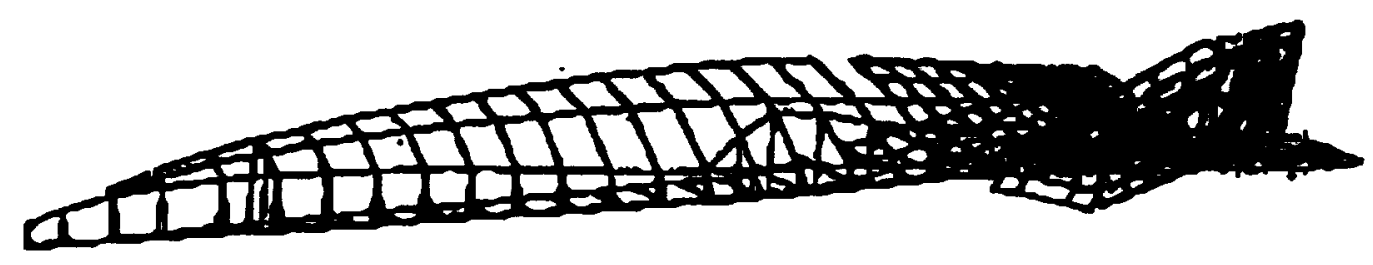

Figure 9. NASTRAN BWB-1 Finite Element Model

reduce the structural degrees of freedom (DOF) of the BWB-1 model from $6000 \mathrm{DOF}$ to 12 generalized (modal) coordinates. At supersonic flight conditions, the vibrating structure couples with the resulting unsteady aerodynamics to cause the modal frequencies and modal damping to change. The unsteady aerodynamic theory used in this study was first order "Piston Theory" (Reference 8) and it was used to generate the aerodynamic influence matrix. The structural equations of motion (EOM) in terms of the generalized or modal coordinates are given in Equation 2-1 of Figure 10. The aerodynamic influence matrix is generated as a function of discrete reduced frequencies $k$. The mass and stiffness matrices are obtained from the NASTRAN modal solution. The structural damping of each mode was assumed to be 0.02 of critical damping. 


\section{Equation 2-1}

$$
\left.[m] \ddot{\bar{x}}_{A}+(1+i g)[K] \bar{x}_{A}=\frac{1}{2} \rho V^{2}[C k)\right] \bar{x}_{A}
$$

where

$$
\begin{array}{rlrl}
\mathrm{m} & =\text { mass matrix } & \rho=\text { freestream density } \\
\mathrm{i} & =\text { complex operator } & \mathrm{V}=\text { freestream velocity } \\
\mathrm{g}=\text { structural damping } & \mathrm{Q}=\text { aerodynamic influence matrix } \\
\mathrm{K}=\text { stiffness matrix } & k=\text { reduced frequency }(\omega \mathrm{b} N) \\
\bar{x}_{\mathrm{A}}= & \begin{array}{l}
\text { structural dynamic mode } \\
\text { of the aircraft in }
\end{array} & \mathrm{b}=\text { reference length } \\
& \text { generalized coordinates } & &
\end{array}
$$

\section{Equation 2-2}

$$
\left[\left[m_{A}\right] s^{2}+(1+i g)[K]-\frac{1}{2} \rho v^{2}\left[Q_{A}(k)\right]\right] \bar{x}_{A}=-\left[\left[m_{A C}\right] s^{2}-\frac{1}{2} \rho v^{2}\left[Q_{A C}(k)\right]\right] x_{C}
$$

where

$$
x_{C}=\text { rigid body control surface mode }
$$

\section{Equation 2-3}

$$
\left\{\frac{\bar{x}_{A}}{x_{C}}\right\}=-\left[\left[m_{A}\right] s^{2}+(1+i g)[K]-\frac{1}{2} \rho v^{2}\left[Q_{A}(k)\right]\right]^{-1}\left[\left[m_{A C}\right] s^{2}-\frac{1}{2} \rho v^{2}\left[Q_{A C}(k)\right]\right]
$$

\section{Equation 2-4}

$$
q_{F, i}=\left[\phi_{q, i}\right]\left\{\frac{\bar{x}_{A}}{x_{C}}\right\} s
$$

where

$q_{F, i}$ is the pitch rate response for the flexible aircraft model at sensor location $i$

$\phi_{q, i}$ is the mode shape for each mode at sensor location $i$

Figure 10. Flexible Vehicle Model Equations 
The initial aeroservoelastic objective is to obtain the response output at the aircraft sensor caused by a flexible airframe due to the aircraft control surface input. Thus, a transfer function with the aircraft sensor response as the output and the aircraft control surface as the input was developed. To obtain the output response, an inertial coupled model of the BWB-1 wing was developed. The wing is an all moving control surface for controlling the BWB-1 aircraft in the pitch axis. The wing inertia and aerodynamic forces are assumed to excite the structure in the pitch axis. The structural response of a sensor is a function of its location on the structure and frequency of excitation. Generally, the structure will have a large response when excited at a frequency that corresponds to a natural frequency of the structure. The structure will also have a maximum response at a structural anti-node point, and a minimum response at a structural node point. The EOM with the rigid control surface mode both aerodynamically and inertially coupled into the system is given by Equation 2-2 of Figure 10.

Equation $1 \mathrm{~b}$ can be manipulated to obtain a transfer function response in terms of the generalized coordinates. The solution to the transfer function equation is made by transforming the equation into the Laplace (or complex frequency) domain. The complex frequency response calculation is performed using Equation 2-3 of Figure 10 by varying the complex frequencies, $s$, over the range of interest. The aerodynamic terms for the flexible aircraft $Q_{A}$ and the control surface $Q_{A C}$ are interpolated for the complex frequency, $s$, of interest.

It should be noted that the Equation 2-3 predicted response is in modal coordinates, and must be transformed to physical coordinates to obtain the response for a sensor at a given aircraft fuselage location. This transformation is made using the NASTRAN mode shapes at the sensor location. This process is mathematically depicted in Equation 2-4 of Figure 10.

As an example, the flexible pitch rate response at sensor location FS 84 is shown in Figure 11. A similar response at FS 1050 is given in Figure 12. Note that the first structural mode occurs at approximately $2 \mathrm{~Hz}$.

After obtaining the response for a given location, the result is transformed into a equivalent state space model using FAMUSS - a MCAIR proprietary technique developed under a MCAIR Independent Research and Development project (Reference 9). 


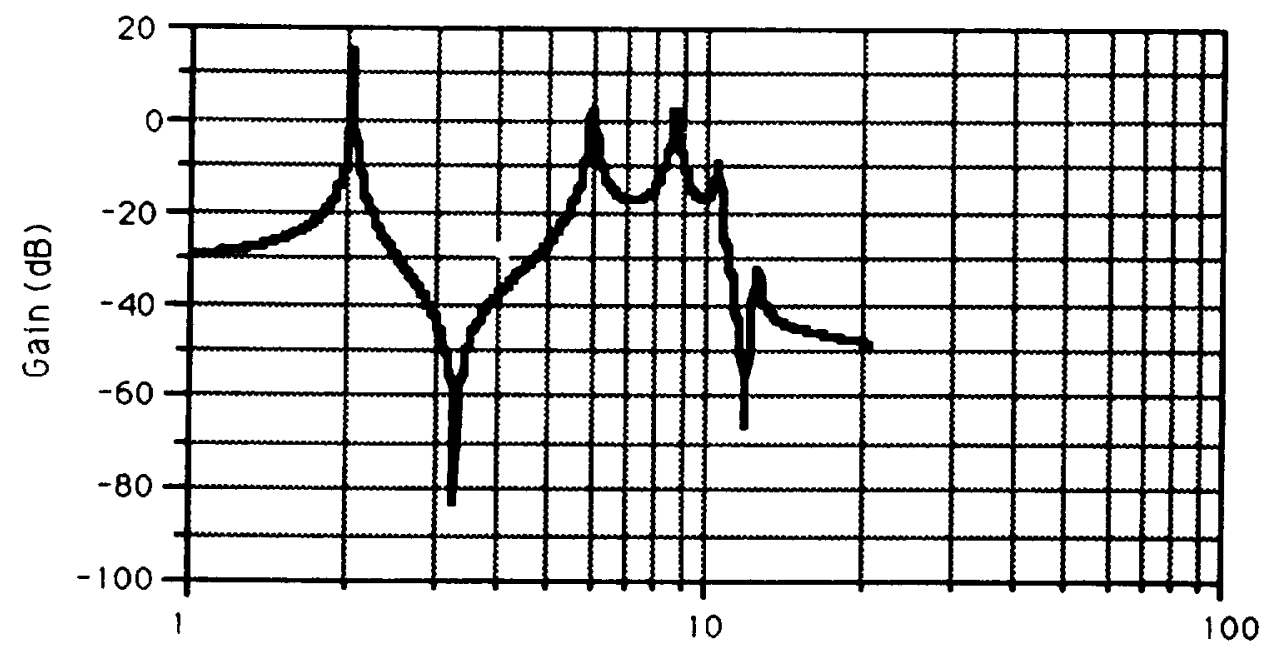

Frequency $(\mathrm{Hz})$

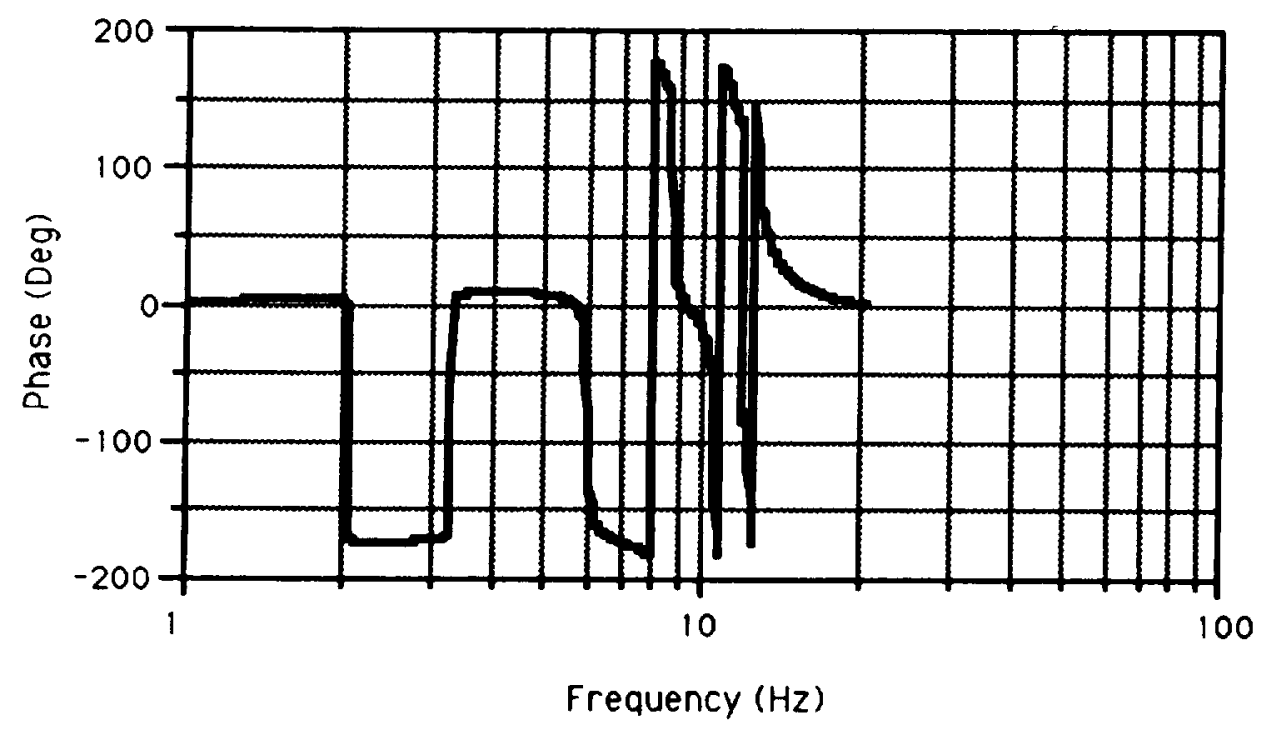

Figure 11. Flexible Pitch Rate Response at FS 84 


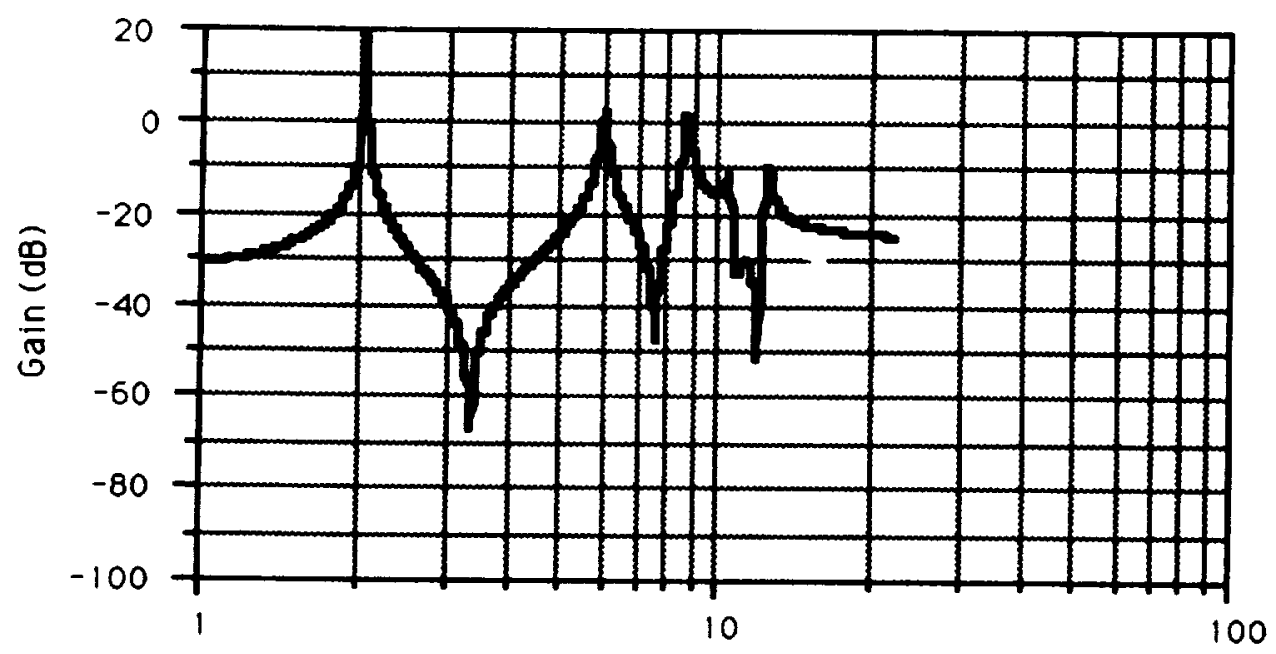

Frequency $(\mathrm{Hz})$

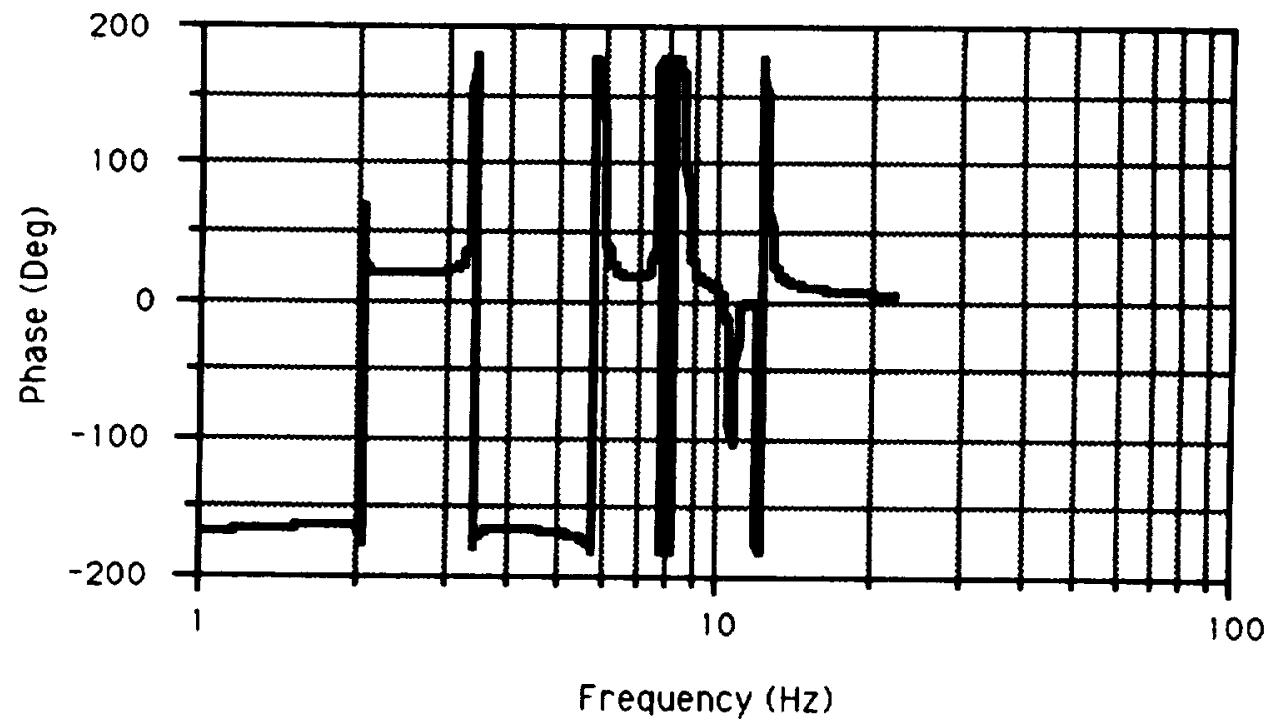

Figure 12. Flexible Pitch Rate Response at FS 1050 


\section{Actuator Dynamics}

Figure 13 shows the actuator dynamic model used in this contract. This actuator is a third order linear model representing the dynamics as installed in current operational aircraft.

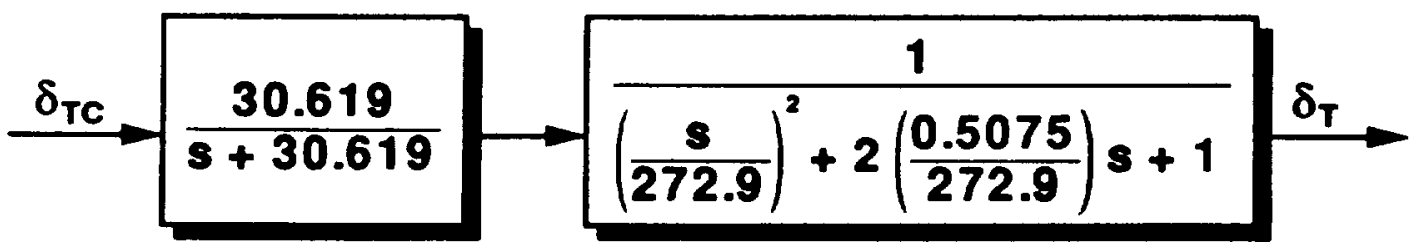

Figure 13. Actuator Dynamics 
This page is intentionally left blank. 


\section{Section III}

\section{FLIGHT CONTROL SYSTEM DESIGN: RIGID BODY LEVEL}

\section{A. Superaugmented Pitch Loop Concept}

The general objective of this contract is to explore the potential of Hybrid Phase Stabilization (HPS) particularly for highly unstable aircraft using HSVs as a relevant example type. Relaxed static stability aircraft must be highly augmented; thus, one of the first items of work is to establish a flight control system architecture. For the purposes of this contract, it is very important that the flight control system development be basic and generic so that conclusions regarding the potential of HPS compared to conventional gain stabilization can be drawn with a maximum of generality. This puts a premium on design procedures which not only lead to good systems, but which give insight into the critical considerations and parameters in the design. Since we are only concerned with longitudinal dynamics, the superaugmented pitch loop (References 10 and 11) is appropriate on all counts.

The fundamentals of the superaugmented pitch loop are summarized in Figure 14. This design creates a pitch rate command, attitude hold (RCAH) characteristic (if the command filter is essentially a pure gain). HSVs are capable of operating at such high speeds that kinematic effects due to the earth's curvature can be significant and, strictly speaking, invalidate the "flat earth" approximation (Reference 12) routinely used in conventional aircraft flight control analysis. In particular, an HSV flying a steady constant altitude, great circle course would hold constant pitch attitude (with respect to the local direction of the gravity vector); however, the pitch rate would not be zero. Consideration of the Figure 14 system shows that the "attitude hold" mode (zero command input) is really zero pitch rate rather than constant attitude. While this is not significant under the flat earth approximation, it is a consideration for HSVs. This issue could be addressed by augmenting the pitch rate feedback with pitch attitude. However, these kinematic effects due to curvature of the earth appear at very low frequencies, below the phugoid, and can be treated separately from the dynamics at mid to high frequencies of interest in this contract. Thus the usual flat earth approximation can be used here. 


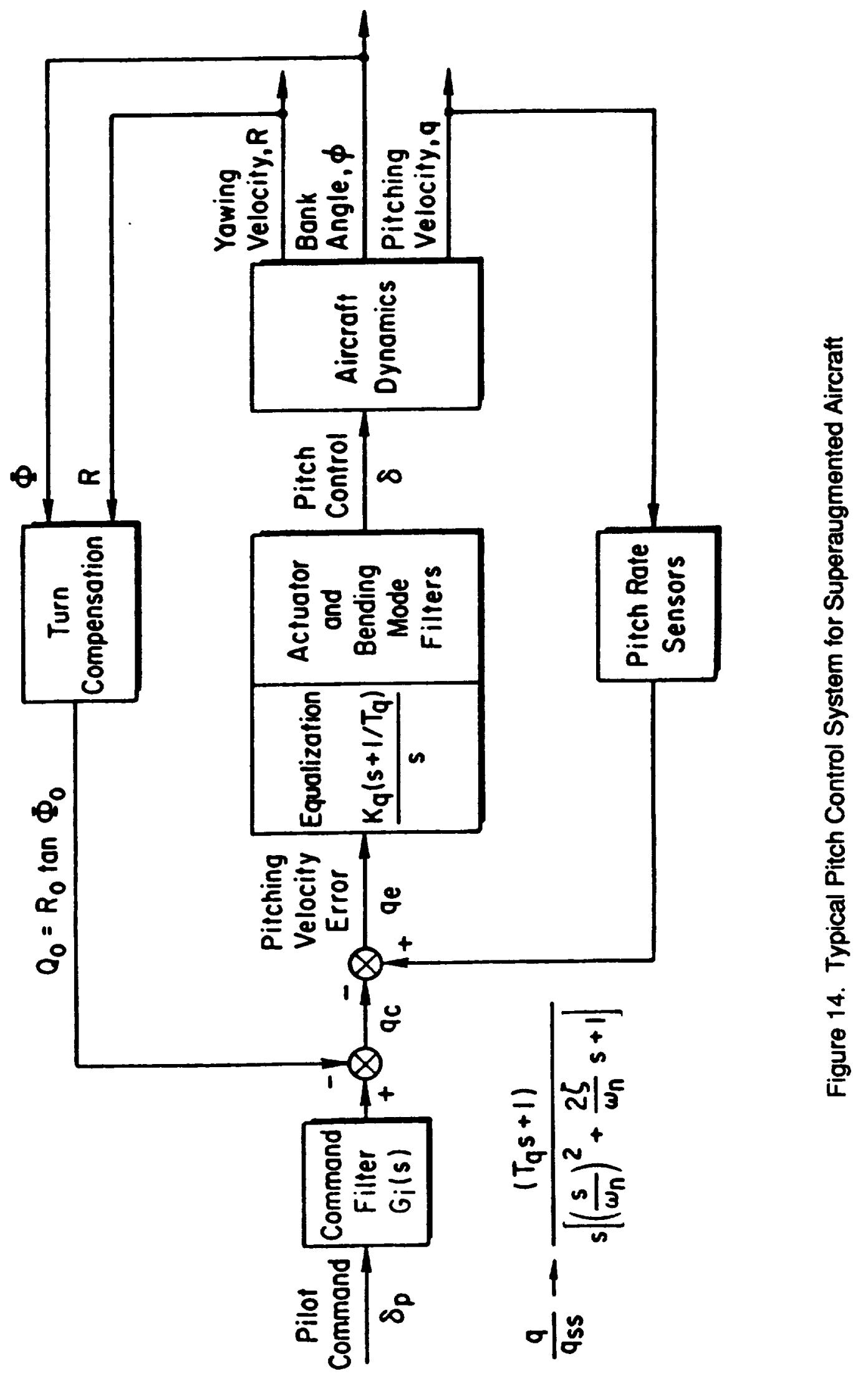


The superaugmented pitch loop can be quite generally satisfactory for "up and away" flight. The only known presently operational HSV, the Space Shuttle, uses this concept. However, to make the results of this study as general as possible, we wish to at least consider the widest range of conceptual FCS types. It is important to distinguish between FCS types and control design methodologies. There are a great profusion of methodologies emerging such as the many variants of $\mathrm{H}_{\infty}, \mu$ synthesis, eigenvector assignment, etc. that differ in the mathematics of synthesis. However, these mathematical differences in methodology can obscure similarities in effective vehicle dynamics imposed by basic physics. To avoid this problem, we can note that a small number of system concepts covers much of the range of practical FCS possibilities. Specifically three response types - pitch rate command, angle-of-attack command and normal load factor command -provide archetypes for a wide range of feasible FCS. Further, as indicated in Figure 15, $\alpha$ command and $n_{z}$ command systems can be most logically developed by adding a feedback loop to a superaugmented pitch inner loop. Thus the superaugmented pitch loop represents a uniquely fundamental structure, widely applicable in flight control and it will be the basic structure for use in this study. Further, this structure can be analyzed by literal procedures that are particularly useful for developing broad understanding.

The starting point of a superaugmented pitch loop design is the pitch rate to "elevator" (the generic pitch control effector) transfer function. Figure 16 summarizes the short period expression for this transfer function. Table 1 summarizes the pitch rate-to-elevator poles and zeros. The dynamics shown are standard for an unstable aircraft. The poles consist of two real short period poles $\left(1 / \mathrm{T}_{\mathrm{sp} 1}\right.$ and $\left.1 / \mathrm{T}_{\mathrm{sp} 2}\right)$, of which $1 / \mathrm{T}_{\mathrm{sp} 2}$ is generally unstable. The example vehicle is quite unstable; and thus both poles approach the square root of $\mathrm{M}_{\alpha}$ in magnitude. The $1 / \mathrm{T}_{\theta 2}$ zero shows the normal correlation with $\mathrm{Z}_{w}$; however, it is unusually low compared to more conventional aircraft. This low value of $1 / \mathrm{T}_{\boldsymbol{\theta}}$ appears to be a distinguishing and problematic characteristic of HSVs which is related to low lift curve slopes at hypersonic speeds.

There are only two basic decisions for the Figure 14 system concept: definition of the crossover frequency $\left(\omega_{c}\right)$, which primarily determines closed loop bandwidth, and placement of the $1 / T_{q}$ lead. Figure 17 presents a system survey sketch of a "standard" superaugmented design (Reference 11) applied to pitch dynamics characteristic of HSVs. The $1 / T_{\mathbf{q}}$ lead is placed above the rigid body dynamics (set by the short period poles). This creates a region of $\mathrm{k} / \mathrm{s}$ slope for the Bode asymptote which provides an ideal region for loop closure. If the crossover frequency is set above $1 / \mathrm{T}_{\mathrm{q}}$, damping ratios above 0.5 for 
- APPROXIMATE OPEN LOOP $n_{z}$ TRANSFER FUNCTION WITH INNER PITCH LOOP CLOSED

$$
\frac{n^{\prime}}{q_{c}} \approx \frac{U_{0} / g}{\left(T_{\theta_{2}} s+1\right)} \frac{q^{\prime} /^{\prime}}{a_{c}} \approx \frac{U_{0} / g}{\left(T_{\theta_{2}} s+1\right)}
$$

- SYSTEM SURVEY SKETCH OF PURE GAIN OUTER LOOP CLOSURE FOR $a$ COMMAND OR $n_{z}$ COMMAND

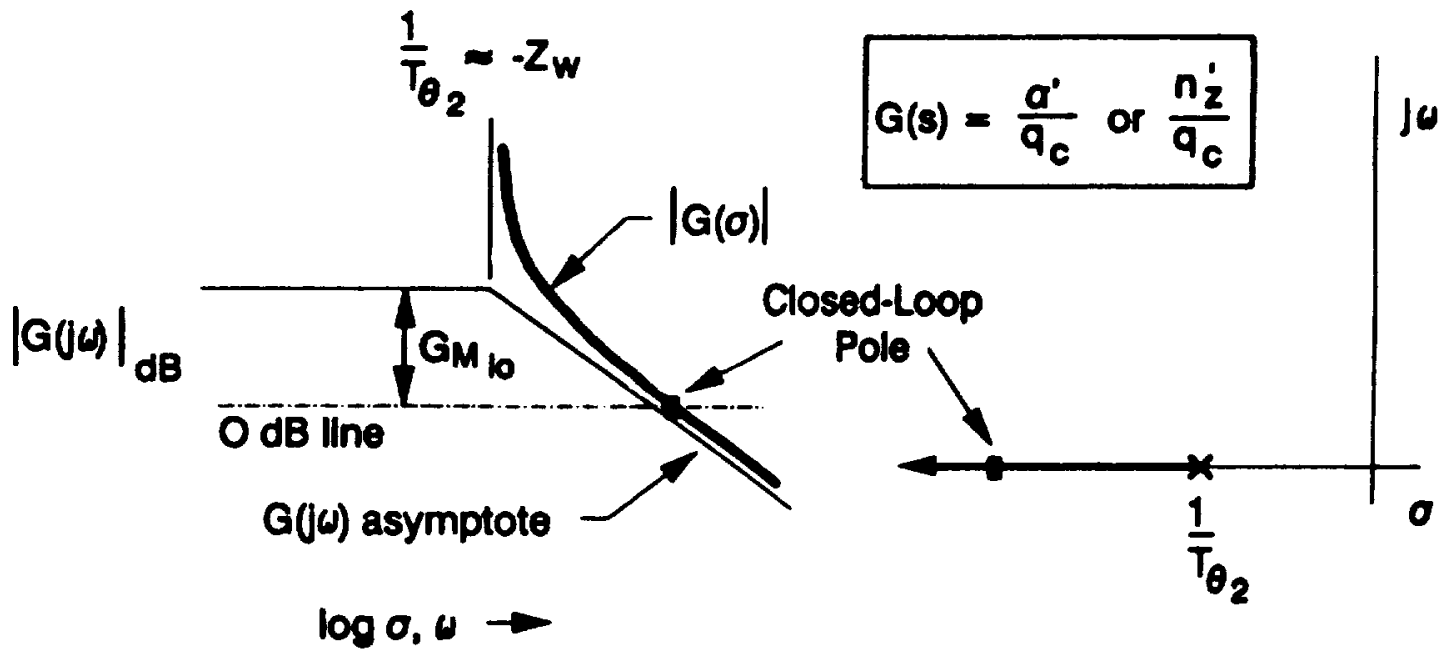

Bode-Slggy Root Locus

Conventional Root Locus

Figure 15. Superaugmented Pitch Loop as Basis for $n_{z}$ and $\alpha$ Command Systems 
Pitch Rate to Control Surface Deflection Transfer Function, $\frac{N_{\delta}^{q}}{\Delta}$

$$
\begin{aligned}
N_{\delta}^{q} & =\left|\begin{array}{cc}
s-Z_{w} & Z_{\delta} \\
-M_{\alpha} & M_{\delta}
\end{array}\right| \quad \text { where } Z_{w}=Z_{\alpha} \\
& =M_{\delta}\left(s-Z_{w}+M_{\alpha} Z_{\delta} / M_{\delta}\right) \\
\Delta & =\left|\begin{array}{cc}
s-Z_{w} & -1 \\
-M_{\alpha} & s-M_{q}
\end{array}\right| \\
& =s^{2}-\left(Z_{w}+M_{q}\right) s+Z_{w} M_{q}-M_{\alpha} \\
& =\left(s+1 / T_{s p 1}\right)\left(s+1 / T_{s p 2}\right)
\end{aligned}
$$

Figure 16. Transfer Function of Short Period Dynamics 


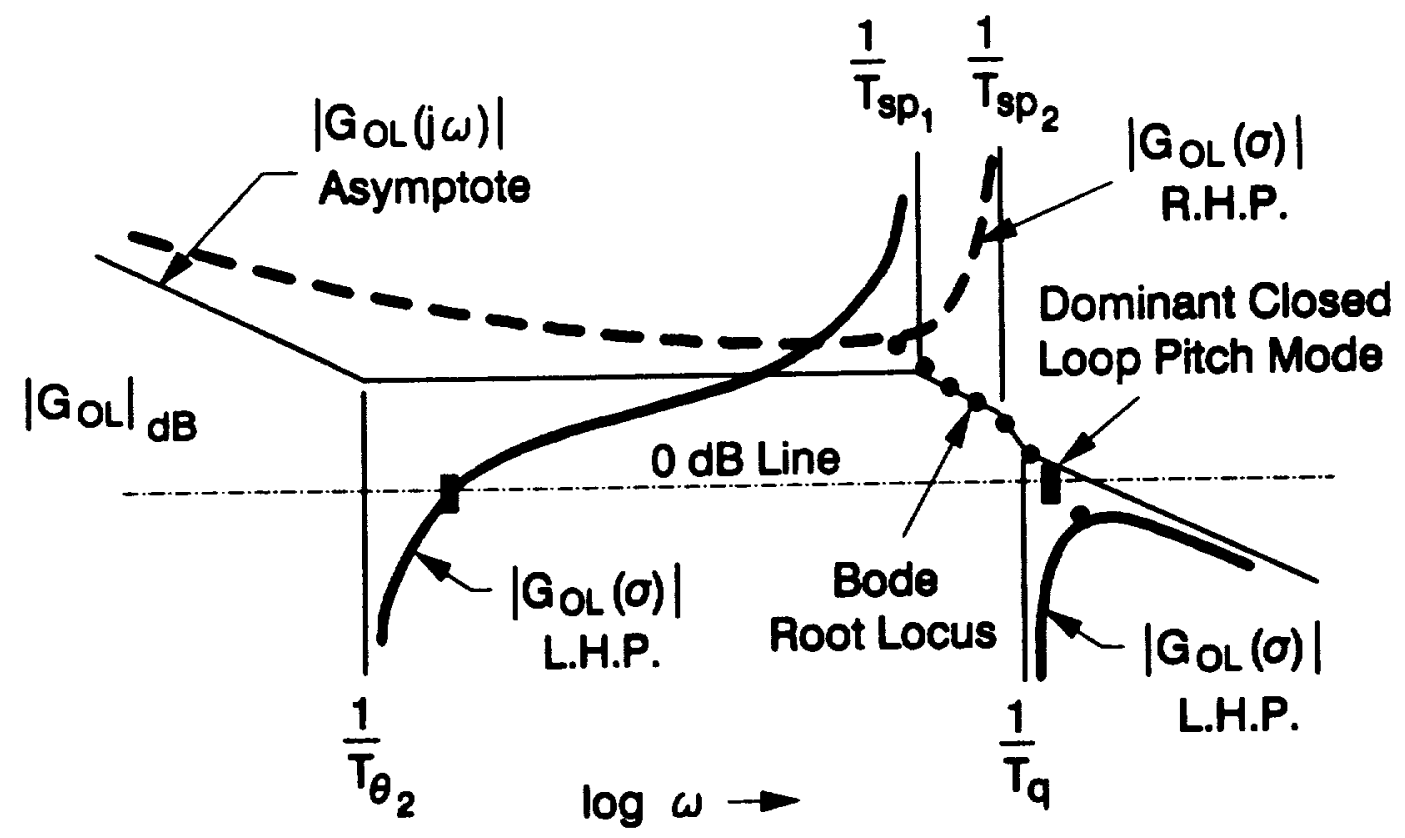

a) Bode-Siggy Root Locus

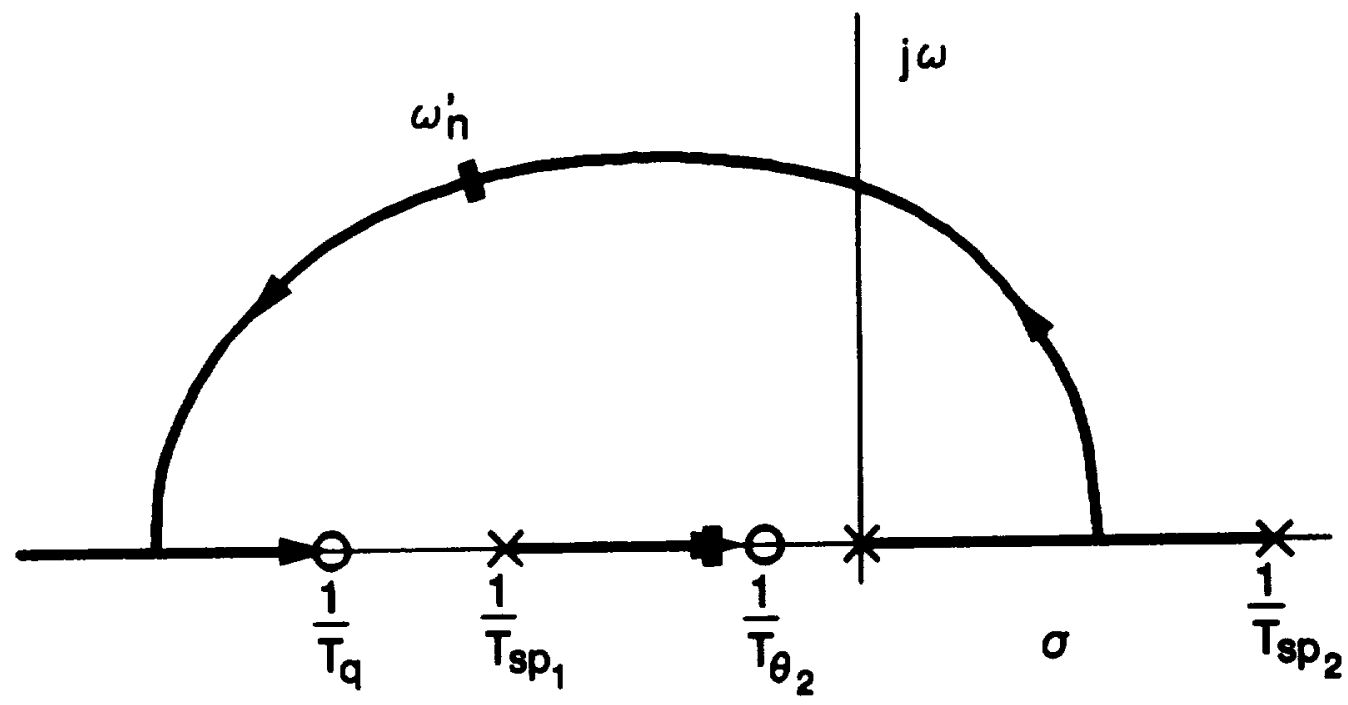

b) Conventional Root Locus

Figure 17. System Survey Sketch of a Superaugmented Design 
the dominant pitch mode would be expected (Reference 11). However, Figure 17 shows that the highly unstable static margin combined with the low hypersonic lift curve slope have resulted in an unusually wide $0 \mathrm{~dB} /$ decade "shelf" between $1 / \mathrm{T}_{\theta 2}$ and the short period poles. This in turn would lead to poor mid frequency gain margin for the standard pitch loop design. This can be solved simply by adding appropriate first order lag-lead compensation to remove the shelf and create the desired broad region of $\mathrm{k} / \mathrm{s}$. Such compensation is straightforward; and ideally, the lag would be placed at $1 / \mathrm{T}_{\theta 2}$ and the lead near the short period poles. These roots do not migrate too far over the Mach range of Table 1; however, this migration could be accommodated by scheduling the lag with estimates of $Z_{W}$ and the lead with estimates of the square root of $M_{\alpha}$.

Assuming ideal lag-lead compensation, the open loop transfer function can be approximated as

$$
G_{\alpha}(s) \approx \frac{K_{q} M_{\delta}\left(1 / T_{q}\right)}{(0)\left(1 / T_{s p_{2}}\right)} e^{-\tau s}
$$

[notation: $(a)=(s+a)$ ]

where an effective time delay $\tau$ has been included as a first order approximation of the high

Table 1. Poles and Zeros of $q / \delta$

\begin{tabular}{|c|c|c|c|c|c|c|c|}
\hline$\gamma$ & Power & Mach & $\sqrt{M_{\alpha}}$ & $1 / \pi_{s p_{1}}$ & $1 / \pi_{s p_{2}}$ & $1 / \pi_{\theta_{2}}$ & $Z_{w}$ \\
\hline Ascent & On & 6 & 2.1543 & 2.305 & -2.0034 & 0.1328 & -0.1180 \\
\hline Ascent & On & 9 & 1.9039 & 2.015 & -1.7929 & 0.1022 & -0.0944 \\
\hline Ascent & On & 15 & 1.9696 & 2.012 & -1.9263 & 0.0415 & -0.0354 \\
\hline Descent & Off & 6 & 3.1496 & 3.308 & -2.9907 & 0.1780 & -0.1380 \\
\hline Descent & Off & 9 & 2.7703 & 2.846 & -2.6946 & 0.0956 & -0.0737 \\
\hline Descent & Off & 15 & 1.9955 & 2.024 & -1.9668 & 0.0340 & -0.0276 \\
\hline
\end{tabular}


frequency (well above the crossover frequency) dynamics including actuators, sensors, computational delays structural dynamics and structural filters.

The basic design decisions can now be reexamined from this simplified open loop transfer function. Figure 18 sketches the asymptotic gain and phase characteristics of this transfer function for $\tau=0$. At this level of approximation, there are three "unalterable" parameters defined by the aircraft configuration -- $M_{\delta}, 1 / T_{s p_{2}}$ and $\tau$. These are considered unalterable in that their determination is significantly influenced or constrained by issues other than control system design. In particular, the effective time delay $\tau$ is determined by control considerations such as actuator bandwidth and, of particular concern here, structural mode control. However, $\tau$ is not a free parameter that can be made arbitrarily small to optimize the control system. The remaining two parameters, crossover frequency and $1 / T_{q}$, are considered the two FCS design variables, but of course they are ultimately subject to constraints as well.

The most fundamental concern is the selection of the crossover frequency (or equivalently the gain $\mathrm{K}_{\mathrm{q}}$ ) because it most directly sets the closed loop bandwidth. The pitch loop bandwidth in turn must be high enough to meet flying qualities requirements for response time and to stabilize the short period mode. The upper limit on loop gain is influenced by the effective time delay -- the smaller $\tau$ is, the higher $\omega_{c}$ can be. However, even if a very small $\tau$ could be achieved, there is still another upper limit on $K_{q}$ due to control power (deflection limits).

\section{B. Impact of High Frequency Dynamics}

A simple approach to superaugmented pitch loop design is presented in Appendix B. This procedure assumes that the high frequency dynamics have negligible impact (i.e., the effective time delay is negligible). This convenient assumption allows the required $\mathrm{K}_{\mathrm{q}}$ and $1 / T_{q}$ to be determined easily for specified values of the dominant mode natural frequency and damping ratio. Appendix $B$ also addresses related issues of sensitivity to aircraft parameter uncertainties, control power and response to command.

However, it cannot be expected that high frequency dynamics can be neglected and, in fact, the impact of HSV structural modes is the focus of this contract. The simple $\tau=0$ design approach provides a basis for addressing the flexibility effects. The first step is to examine these effects with the simplest model -- a nonzero time delay. Inclusion of 


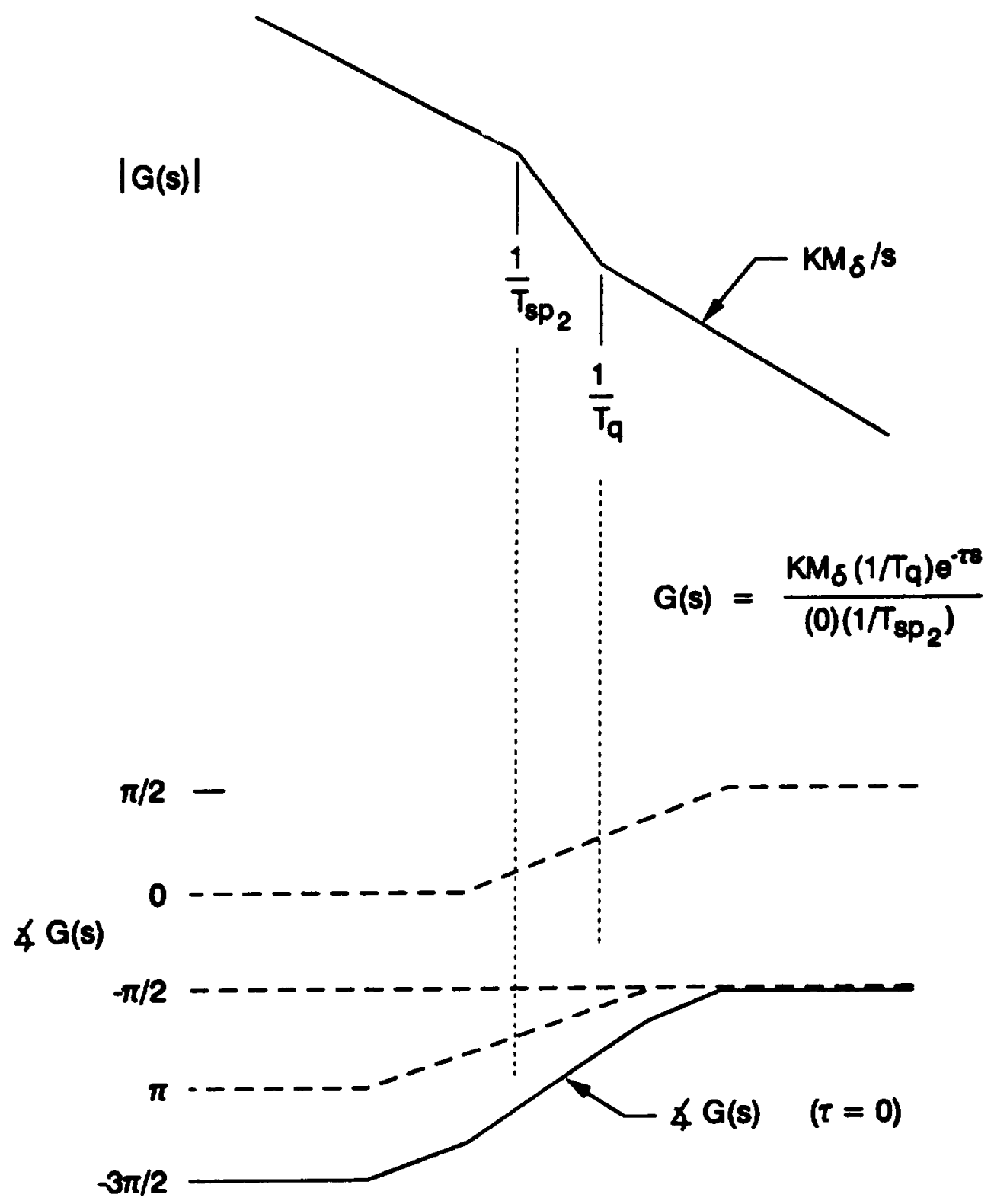

Figure 18. Asymptotic Frequency Response Sktch of the Compensated Open Loop Transfer Function 
effective time delay complicates the Appendix B analysis, and simple literal relationships are not easily obtained. Thus this effect will be examined numerically for the example flight condition (Mach 6, power on, ascent). Figure 19 shows a family of root loci parameterized with the effective time delay $\tau$, holding all other parameters the same for each loci. The loci are computed using third order Pade' approximations of the time delay. The square boxes in Figure 19 indicate the location of the closed loop poles at the nominal loop gain of $\mathrm{K}_{\mathrm{q}}=-1.647 \mathrm{r} / \mathrm{r} / \mathrm{s}$. The complex pole in all cases is the dominant closed loop pole. At high values of time delay, a third real pole appears at low frequencies to further complicate the dynamics. The primary concern, however, is how rapidly the dominant mode deteriorates with increasing time delay above about $70 \mathrm{msec}$.

To put the $\tau$ values of Figure 19 in perspective, it is useful to compare these to values for actual aircraft. Table 2 provides such data. The first four aircraft are fighters and the last is the Space Shuttle. The shuttle is perhaps the most relevant to HSVs and its $\tau$ value of approximately $174 \mathrm{msec}$ would be totally unacceptable for the Figure 19 design. Certain qualifications need to be made regarding the time delay values in Table 2 . These are estimated from the listed component contributions obtained from block diagram examinations. Time delay values obtained from actual frequency responses can be expected to be somewhat lower. In fact, the actual total time delay values for the first four aircraft are less than $100 \mathrm{msec}$. Such comparisons for the shuttle are given in Reference 11. The implication of Figure 19 and Table 2 is that high frequency dynamics must be considered carefully in HSV flight control design.

There is a final point that should be noted in conjunction with Table 2. The ABICS and F15E aircraft contain lead-lag filters for which the effective time delay contribution is determined as negative. This occurs because these elements appear in the feedback loop and reduce the effective time delay, but they are not high frequency "parasitic" lags as are the other components. The lead-lag filters represent compensation filters inserted in the loop to provide lead in the crossover region. In fact, they represent an alternative approach to the hybrid phase stabilization concept considered in this contract. These filters, in effect, are used to estimate derivatives of sensor outputs. Thus this approach is based on using estimation techniques to extend the use of a given set of sensors. In contrast, the hybrid phase stabilization concept is based on the use of additional measurements as opposed to increased estimation. Practical designs may well need both concepts, but HPS is the focus of this study. 


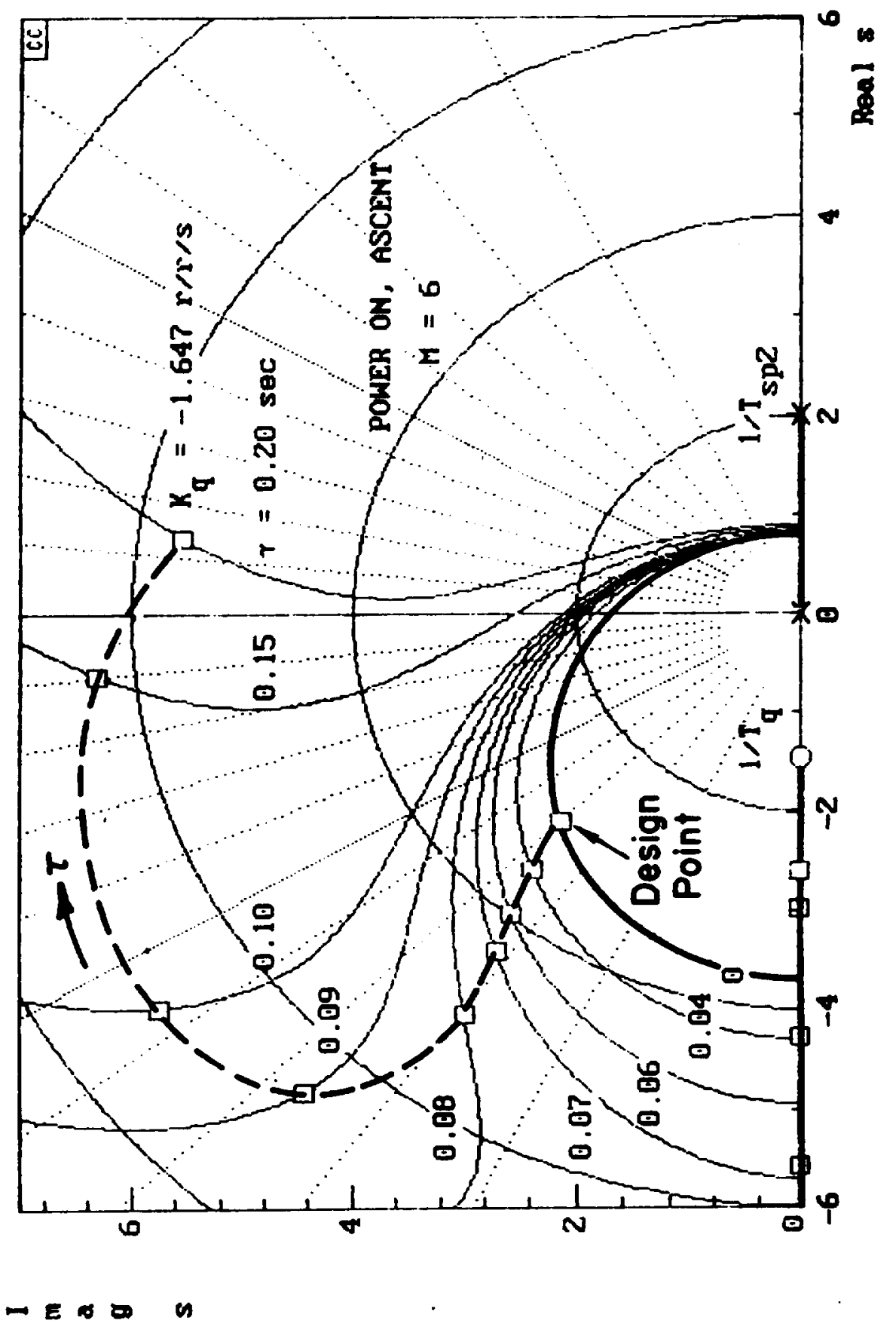

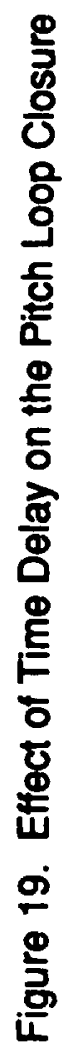


Table 2. "Approximate"1 Time Delay ${ }^{2}$ Survey of Operational Aircraft

\begin{tabular}{|c|c|c|c|c|c|}
\hline AIRCRAFT & ABICS $^{4}$ & F15E & S/MTD $^{5}$ & $F 18$ & $\begin{array}{c}\text { Space } \\
\text { Shuttle }\end{array}$ \\
\hline Actuator & 54.4 & 54.4 & 36.4 & 32.1 & 50.0 \\
\hline Computational Delay & 8.7 & 8.7 & 8.7 & 8.7 & 46.0 \\
\hline Anti-aliasing Filters & 9.9 & 7.8 & 8.9 & 0.5 & - \\
\hline Structural Filters $_{\text {Lead/Lag Filters }}{ }^{3}$ & 45.7 & 41.0 & 50.0 & 62.3 & 78.0 \\
\hline Lost-filter $^{-108.5}$ & 4.2 & 4.1 & 3.9 & 3.4 & - \\
\hline Pure Time Delay & 3.3 & - & - & - & - \\
\hline Total Time Delay & 126.2 & 116.0 & 107.9 & 107.0 & 174.0 \\
\hline
\end{tabular}

1 Time delay "approximation" of operational aircraft. The actual time delay values are smaller and meet the MIL-spec requirements.

2 in milliseconds

3 leadlag filters were not included in the total time delay because they were primarily introduced as equalization for compensating time delay of the system

4 Ada-Based Integrated Control System

5 sTOL Maneuver Technology Demonstrator

\section{Pitch Response Bandwidth Requirement}

The sensitivity to effective time delay increases with the pitch loop bandwidth, and thus definition of the required bandwidth is an important issue for HSVs. This is true of course for any aircraft. Bandwidth criteria have been established (References 13 and 14) for more conventional aircraft. For HSVs in hypersonic flight, there are no firmly established criteria and precedents, and little data is available although research in these 
areas is underway. The Space Shuttle pitch rate step response criteria (Figure 20) are perhaps the best points of reference. The bandwidth requirement is very closely related to the rise time requirement, which in turn corresponds to the initial portion of the lower boundary in Figure 20 . On this basis, the shuttle spec implies a significantly lower bandwidth is acceptable at hypersonic speeds as compared to subsonic flight. However, this must be tempered with the knowledge that next generation hypersonic aircraft can be expected to have more stringent hypersonic maneuvering requirements than the shuttle.

With this caveat in mind, the Figure 20 specs provide a means for connecting to more recently developed pitch rate bandwidth requirements. Figure 21 shows tentative pitch attitude bandwidth requirements proposed for NASP for low speed (approach and landing). Figure 22 summarizes the pertinent definitions underlying this bandwidth specification. By this criterion, the nominal system of Figure 19 has a bandwidth of $3.8 \mathrm{rad} / \mathrm{sec}$ with no time delay (which corresponds to essentially zero phase delay as well). Thus, this system would be well into the "desired" region of Figure 21. For the examples that follow, a lower Level 1 bandwidth value of $2.0 \mathrm{rad} / \mathrm{sec}$ will be used. It will be seen that even this reduced bandwidth requirement creates significant difficulties for flight control design of a flexible HSV.

The superaugmented pole placement formulas above can be combined with the bandwidth definitions of Figure 22 to define the loop parameters from a specified bandwidth (see Figure 23). Table 3 shows an application of the Figure 23 iterative procedure for the example case.

The time delay sensitivity survey corresponding to Figure 19 is shown in Figure 24 for the lower bandwidth design. It can be seen that the bandwidth reduction has reduced the time delay problem somewhat, but the potential problem is still significant.

\section{The Baseline System}

To further address high frequency dynamics, we must compare FCS designs applied to the actual flexible aircraft. This will be done in the next two sections; first, for a conventional gain stabilized design, and then for the hybrid phase stabilized design. However, as an additional "baseline" reference case (but not a true FCS design), the superaugmented pitch loop (with $2 \mathrm{rad} / \mathrm{sec}$ bandwidth) will be directly applied to the flexible aircraft without structural compensation. The frequency response of the open loop 


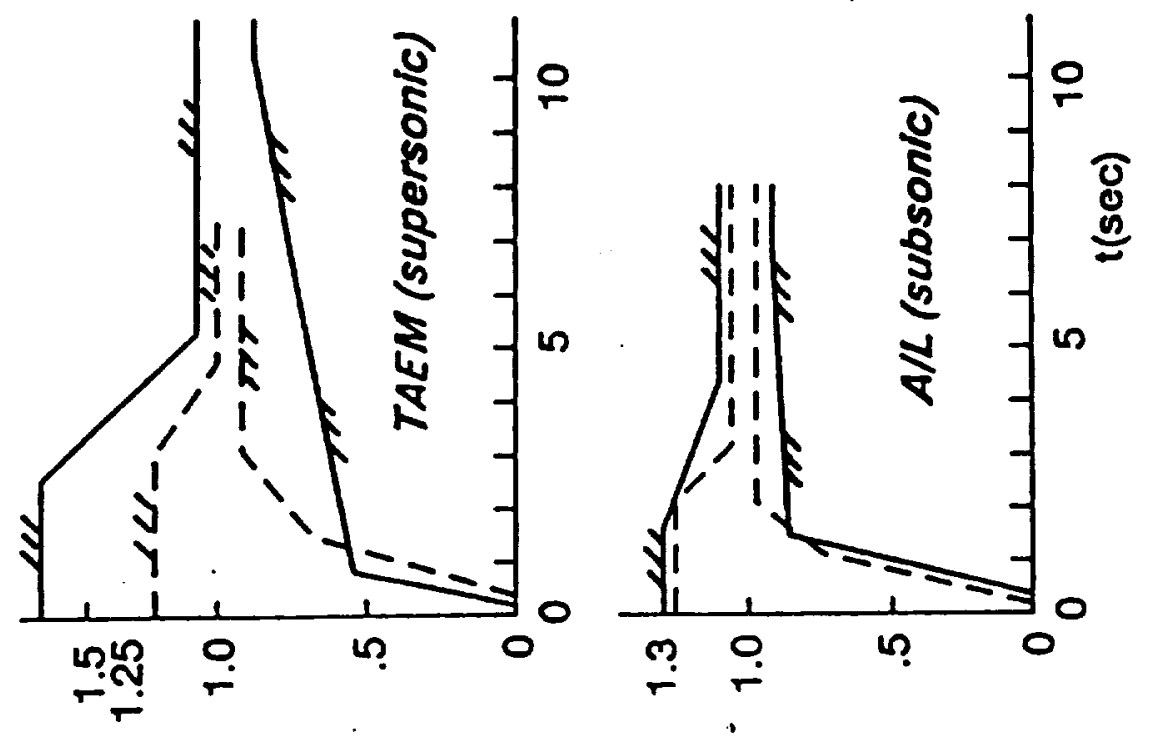

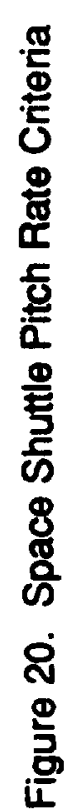
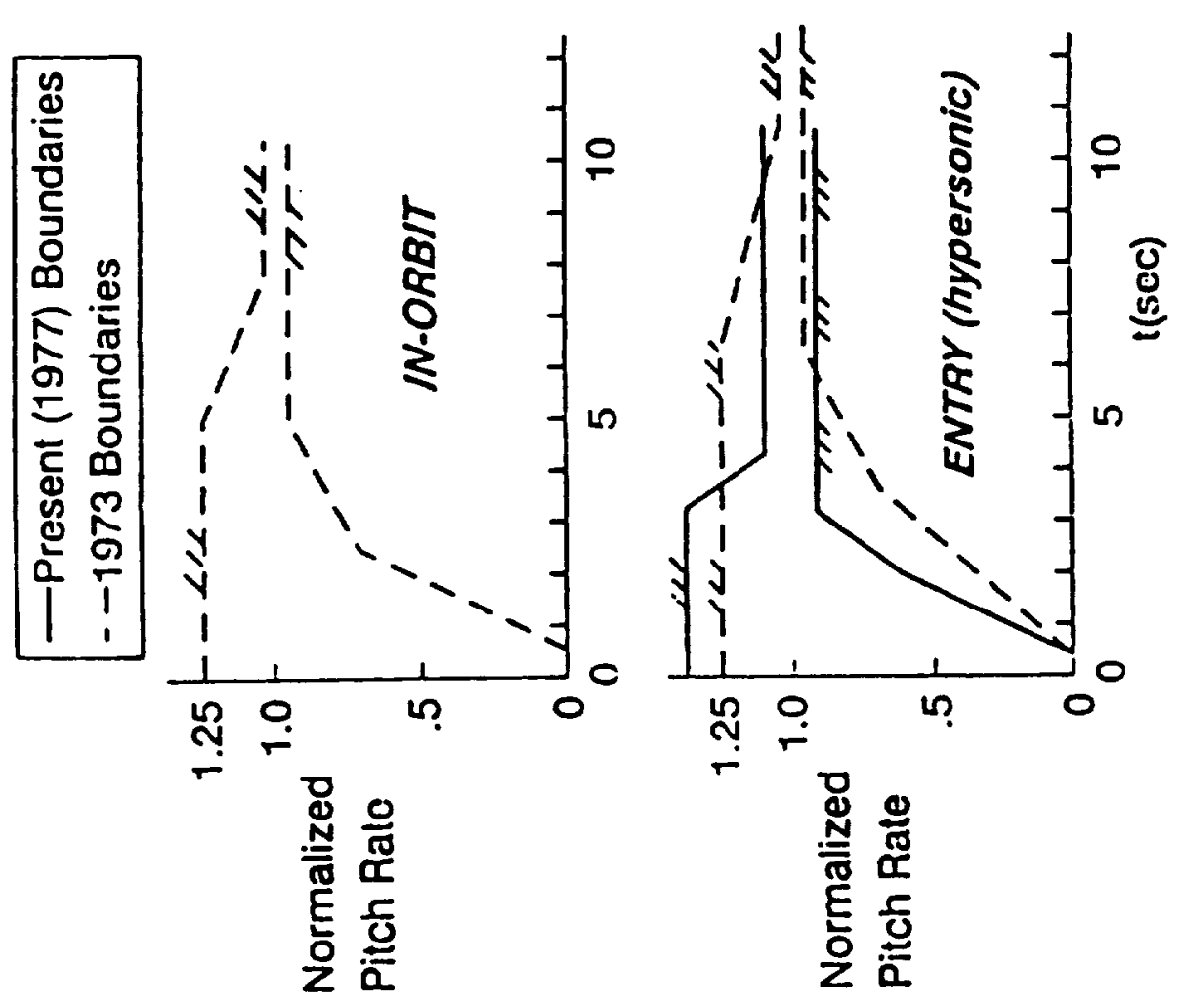


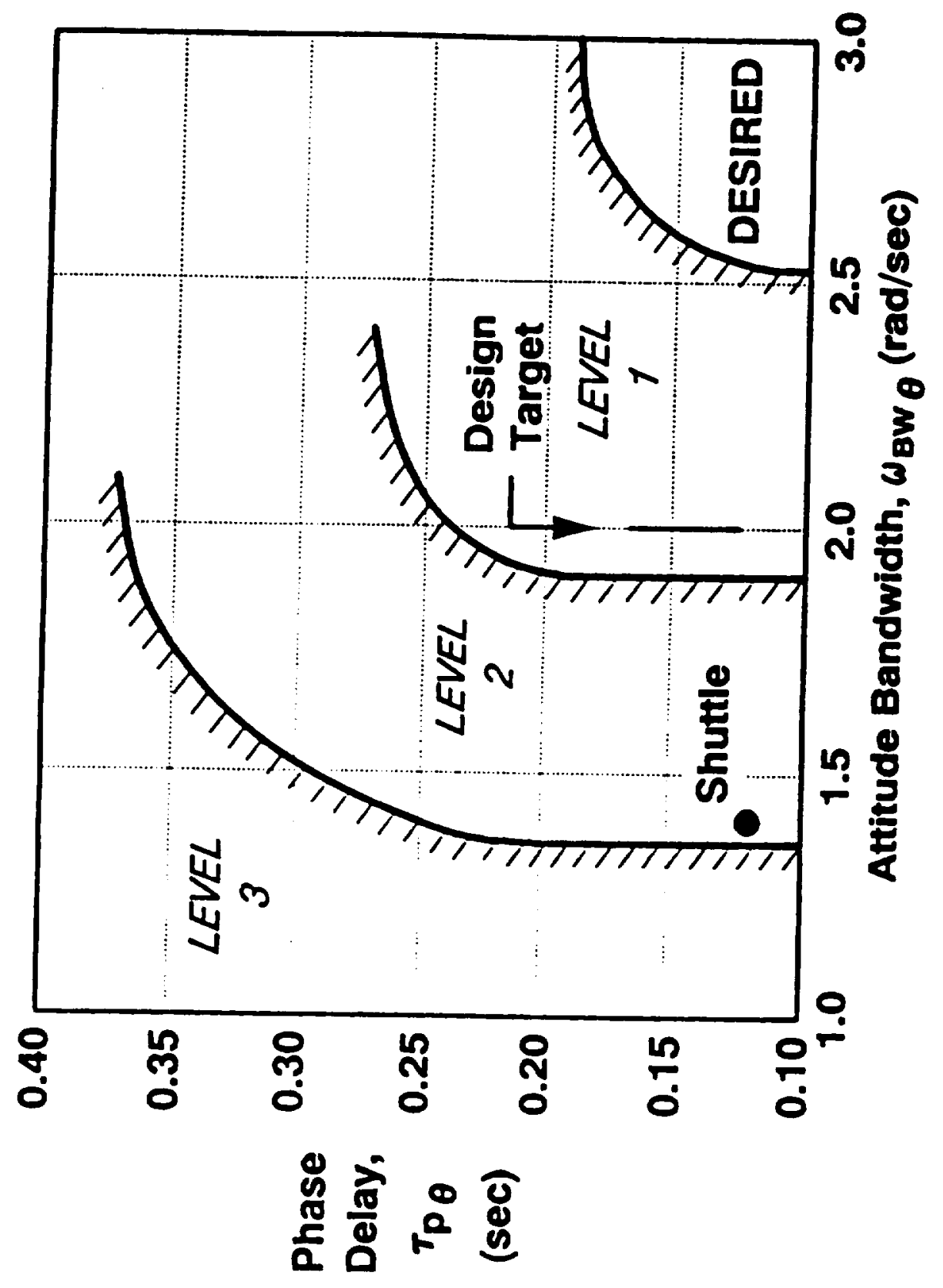

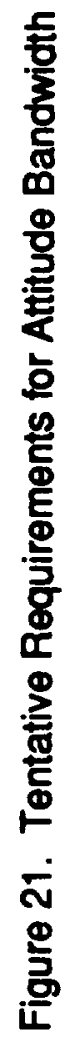


Phase Delay:

$$
\tau_{p}=\frac{\Delta \Phi 2 \omega_{180}}{57.3\left(2 \omega_{480}\right)}
$$

Rate Response-Types:

$\omega_{B W}$ is lesser of $\omega_{B W_{\text {gain }}}$ and $\omega_{B W_{\text {phase }}}$

Attltude Response-Types (including GCGH):

$\omega_{\mathrm{BW}}=\omega_{\mathrm{BW}} \mathrm{whese}$
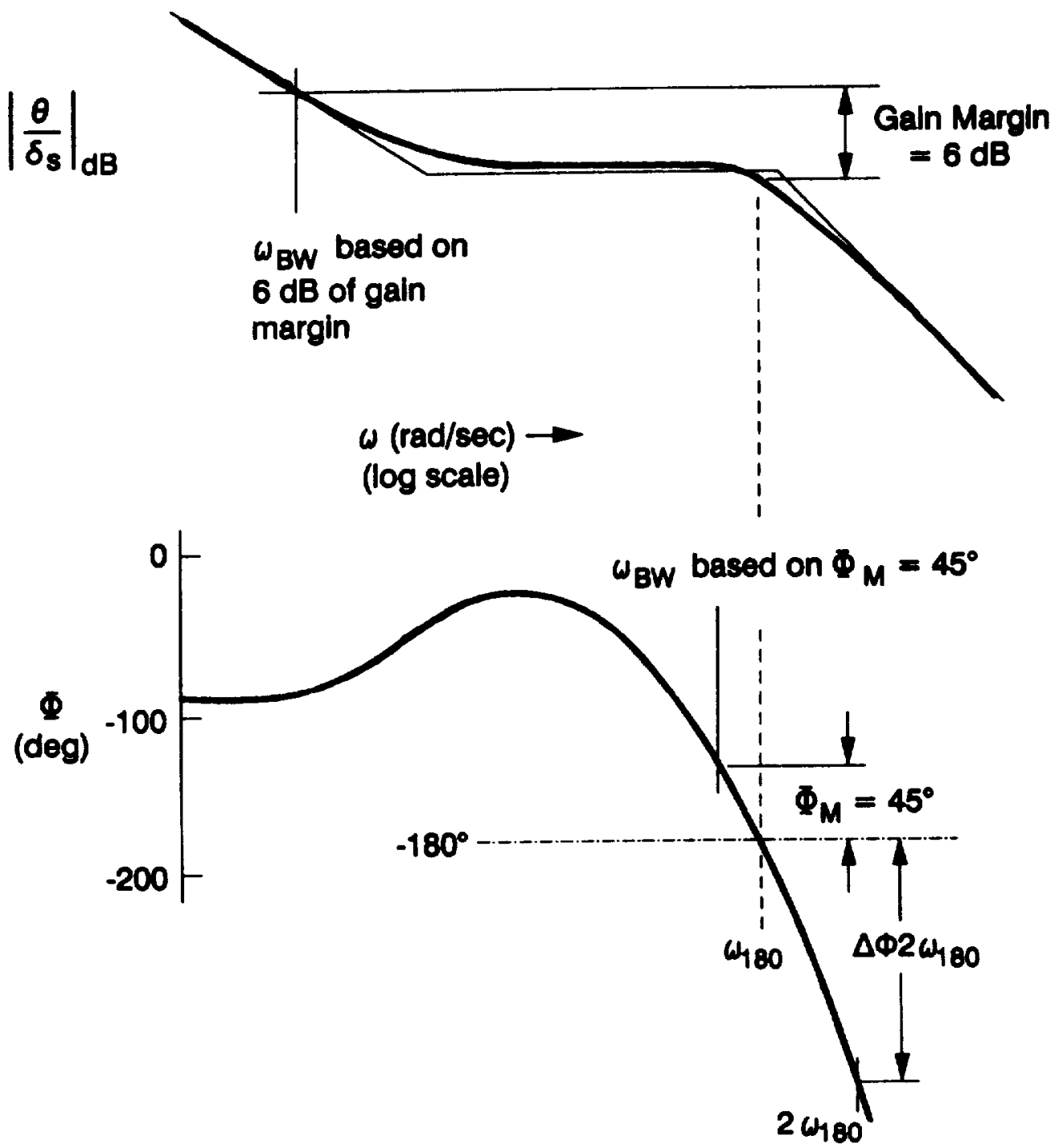

Figure 22. Measurement of Airplane Bandwidth, $\omega_{\mathrm{BW}}$ 
- Transfer Function o involved (6e0 Appendix B)

$$
\begin{aligned}
& G=\frac{q}{\delta}=\frac{M_{\delta}\left(1 / T_{\theta_{2}}\right)}{\left(1 / \pi_{\theta p_{1}}\right)\left(1 / T_{\theta p_{2}}\right)} \quad G_{c}=\frac{\delta}{q_{E}}=\frac{K_{q}\left(1 / T_{q}\right)\left(1 / \hat{T}_{s p_{1}}\right)}{(0)\left(1 / \hat{T}_{\theta_{2}}\right)} \\
& G_{C L}=\frac{q^{\prime}}{q_{c}}=\frac{K_{q} M_{\delta}\left(1 / \pi_{q}\right)}{\left[\zeta, \omega_{n}\right]}
\end{aligned}
$$

- Assumptions

$$
\begin{aligned}
& -\tau_{\theta}=0 \\
& -\left(1 \hat{T}_{s p_{1}}\right)=\left(1 / \pi_{t p_{1}}\right) \\
& -\left(1 \hat{T}_{\theta_{1}}\right)=\left(1 / \pi_{\theta_{2}}\right) \\
& -5=0.7
\end{aligned}
$$

- Design Sequence from Flying Qualities Spec

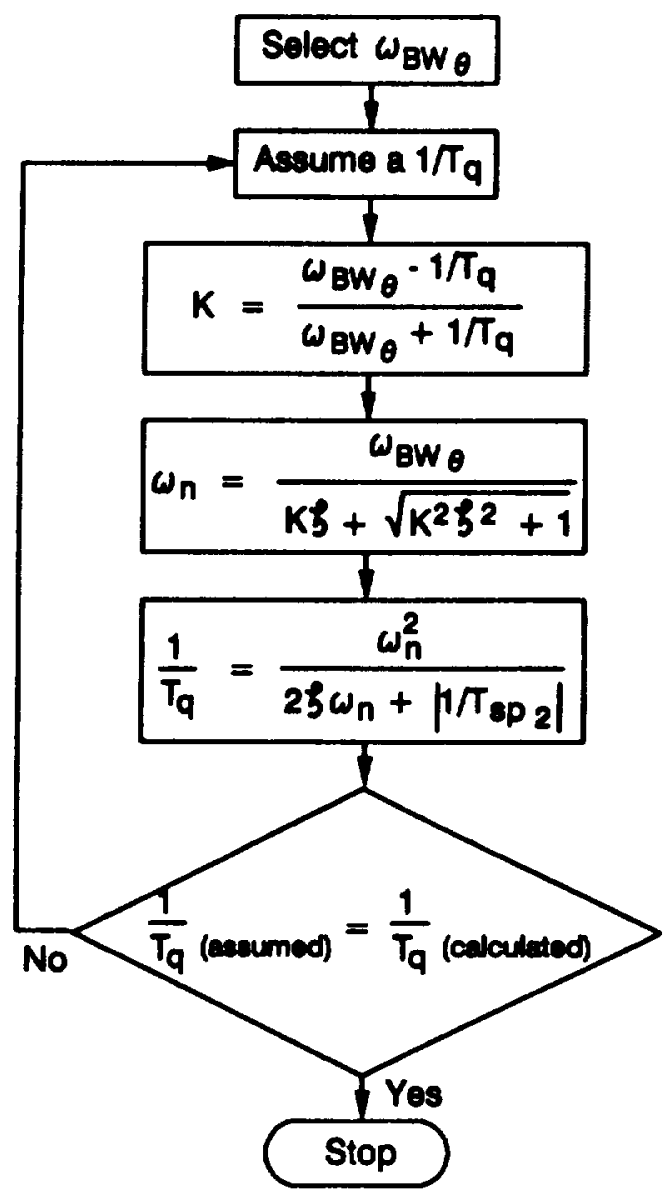

Figure 23. Superaugmented Pitch Loop Design to a Specified Pitch Loop Bandwidth

35 
Table 3. Design of Pitch Loop to Specified Bañdwidth

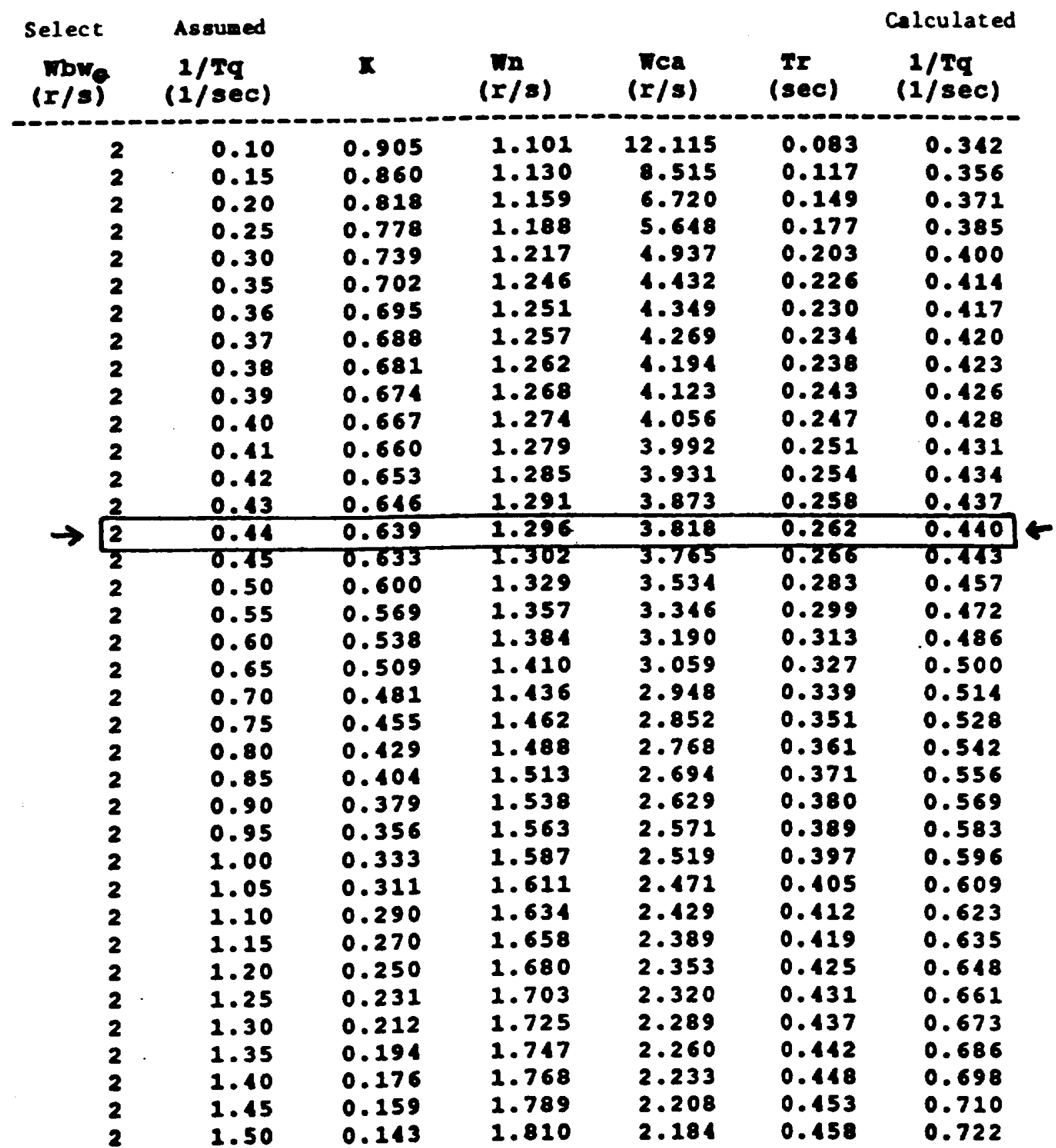




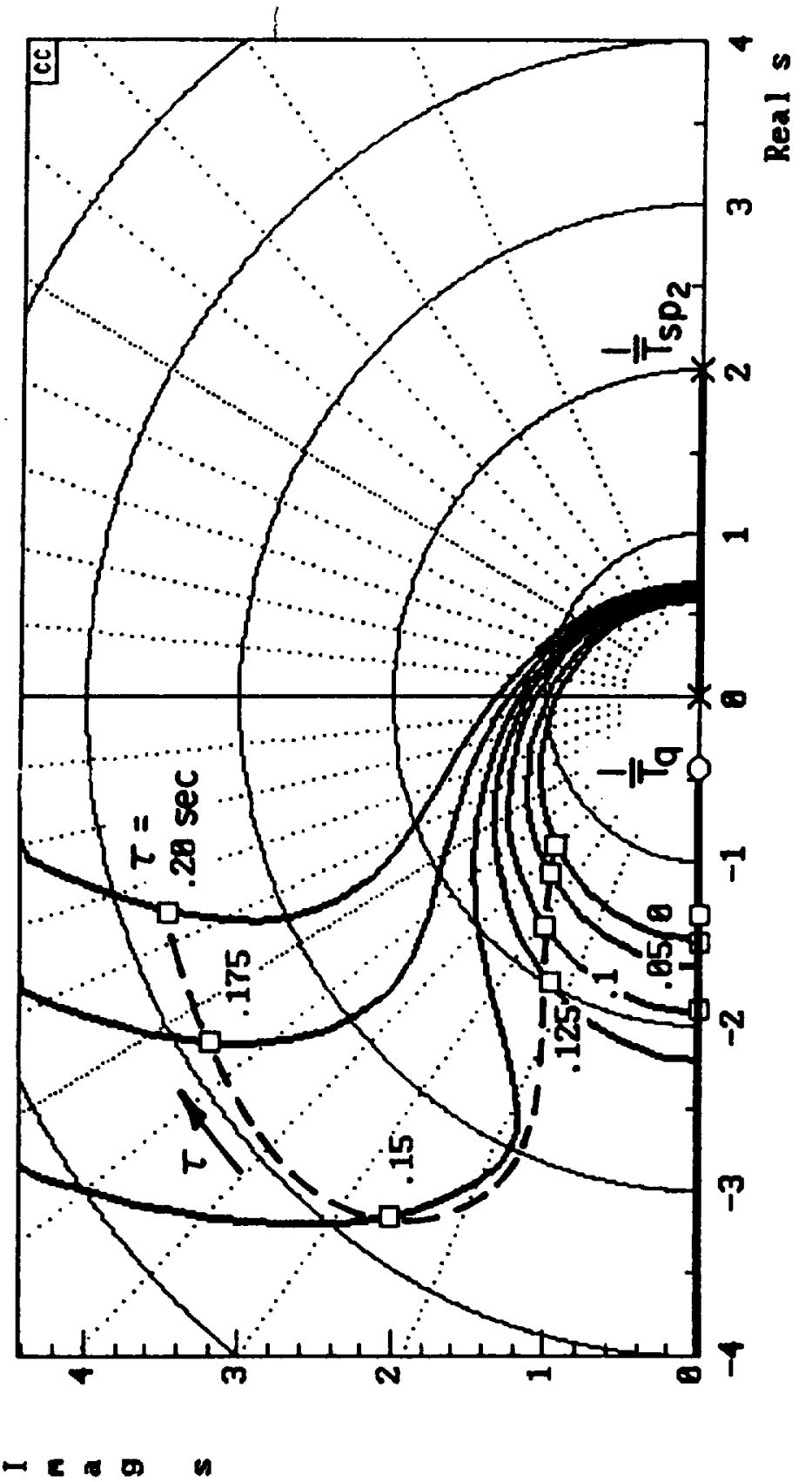

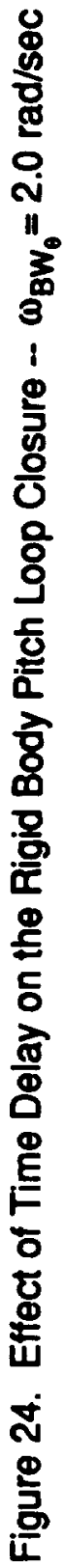


transfer function is shown in Figure 25. The signal is obtained from the forward sensor position (FS 84). The five structural modes appear as peaks in the Bode magnitude plot. The first three structural modes clearly do not meet the gain margin requirements of the MIL-spec reviewed in Section I-B.

In the mid frequency region below the structural modes, the phase margin is 42 degrees at the magnitude crossover (just above $3 \mathrm{rad} / \mathrm{sec}$ ) which corresponds to a delay margin of $0.223 \mathrm{sec}$. The phase angle steadily decreases as the frequency decreases below the crossover. This is consistent with the conditional stability of the superaugmented pitch loop. The gain margin corresponding to the low frequency phase crossover near $1 \mathrm{rad} / \mathrm{sec}$ is only about $5 \mathrm{~dB}$. These gain and phase margins are also somewhat less than the MIL-spec requirements.

Looking ahead, it is important to note that these lower frequency "rigid body" margins will be further degraded by the treatment of the structural modes. This is of course the motivation for the hybrid phase stabilization concept as an alternative to the conventional gain stabilization method. Within the scope of this contract, only the incremental improvement of the HPS concept over the conventional approach can be addressed. It should be kept in mind in the following developments that further mid frequency compensation, i.e., estimation based approach as noted in Section III-B, would also be used to meet the margin requirements in the mid frequency region. These will be assumed in the following developments, but correction of the mid frequency margins will not be considered further as we focus now on the higher frequency structural modes.

The characteristics of the baseline (or the uncompensated) system can be further investigated from the system survey of Figure 26. The conventional root locus plot shows that the second, third and fifth structural modes potentially can be driven unstable. The Bode root locus plot (Reference 9) makes the gain sensitivity and margins much clearer (and thus this plot will be used routinely). On this plot, complex loci appear as dotted lines; the dots are solid where the locus lies in the left half s plane and open in the right half plane. From the Bode loci of Figure 26, it can be readily seen that the first structural mode can, in principle, be driven unstable as well, but more importantly the third, followed by the first, mode has the lowest stability margin. Careful comparison of the crossover region with that of Figure B-6 in Appendix B shows that the two are very similar. 


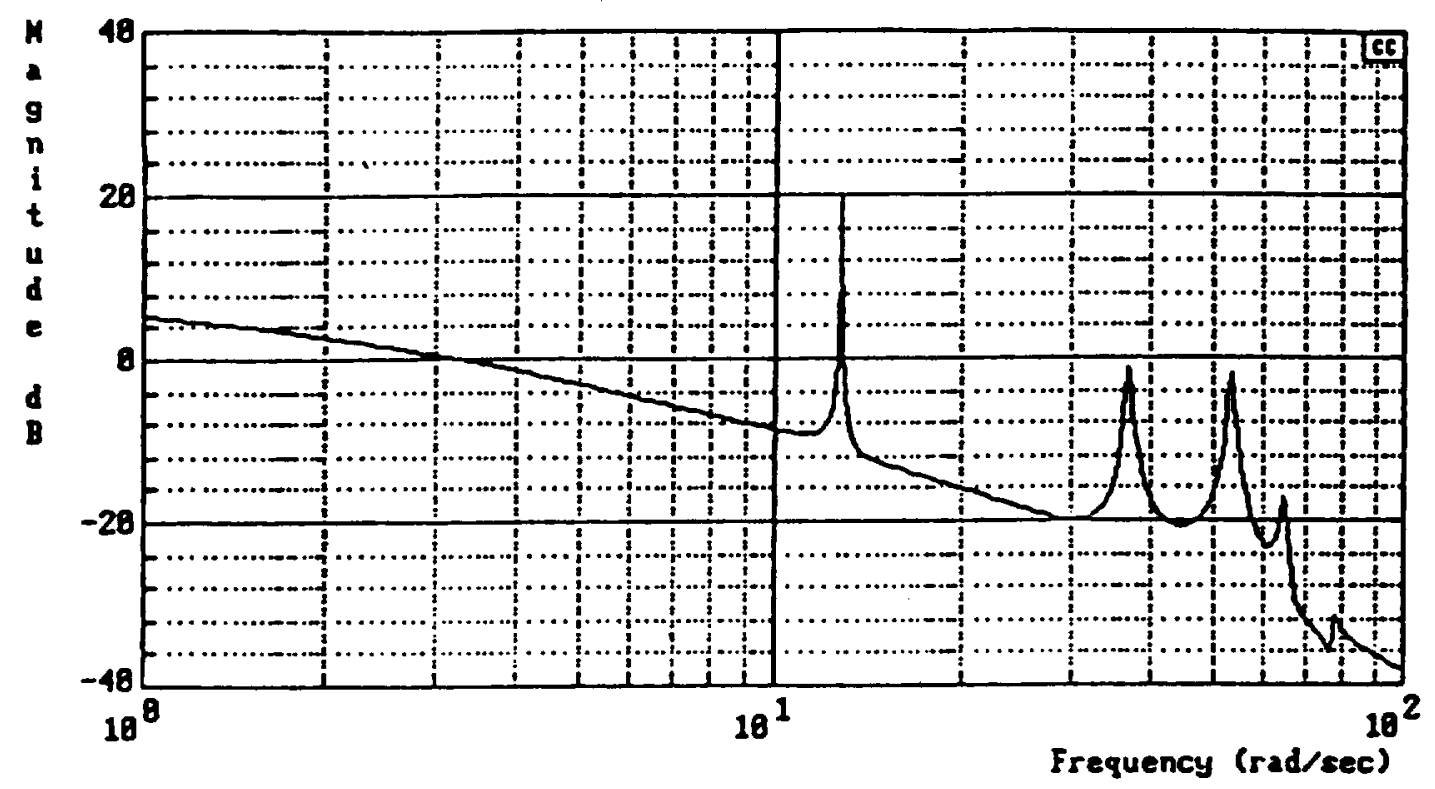

Bode Magnitude Plot

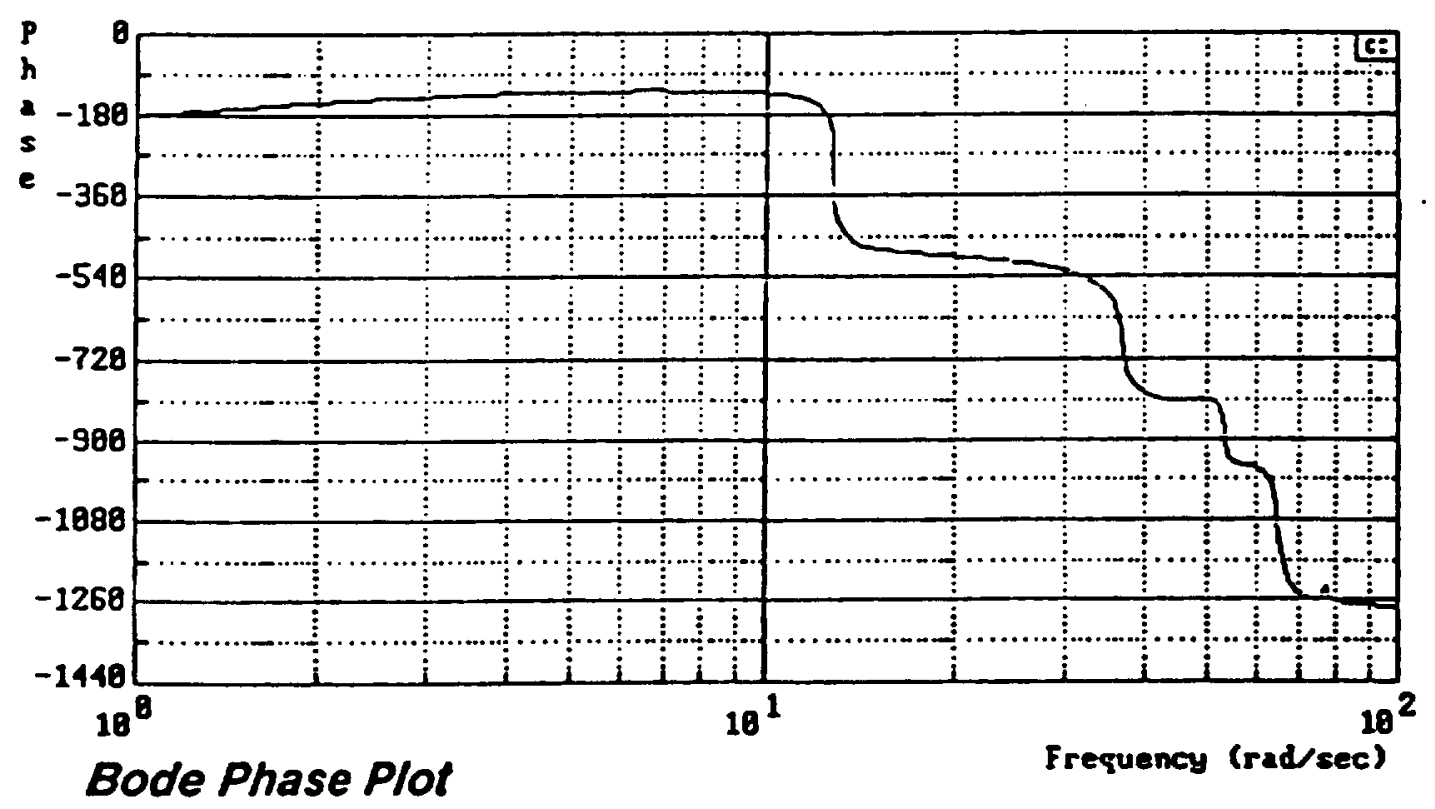

Figure 25. Baseline System Open loop Frequency Response - Ascent Case 


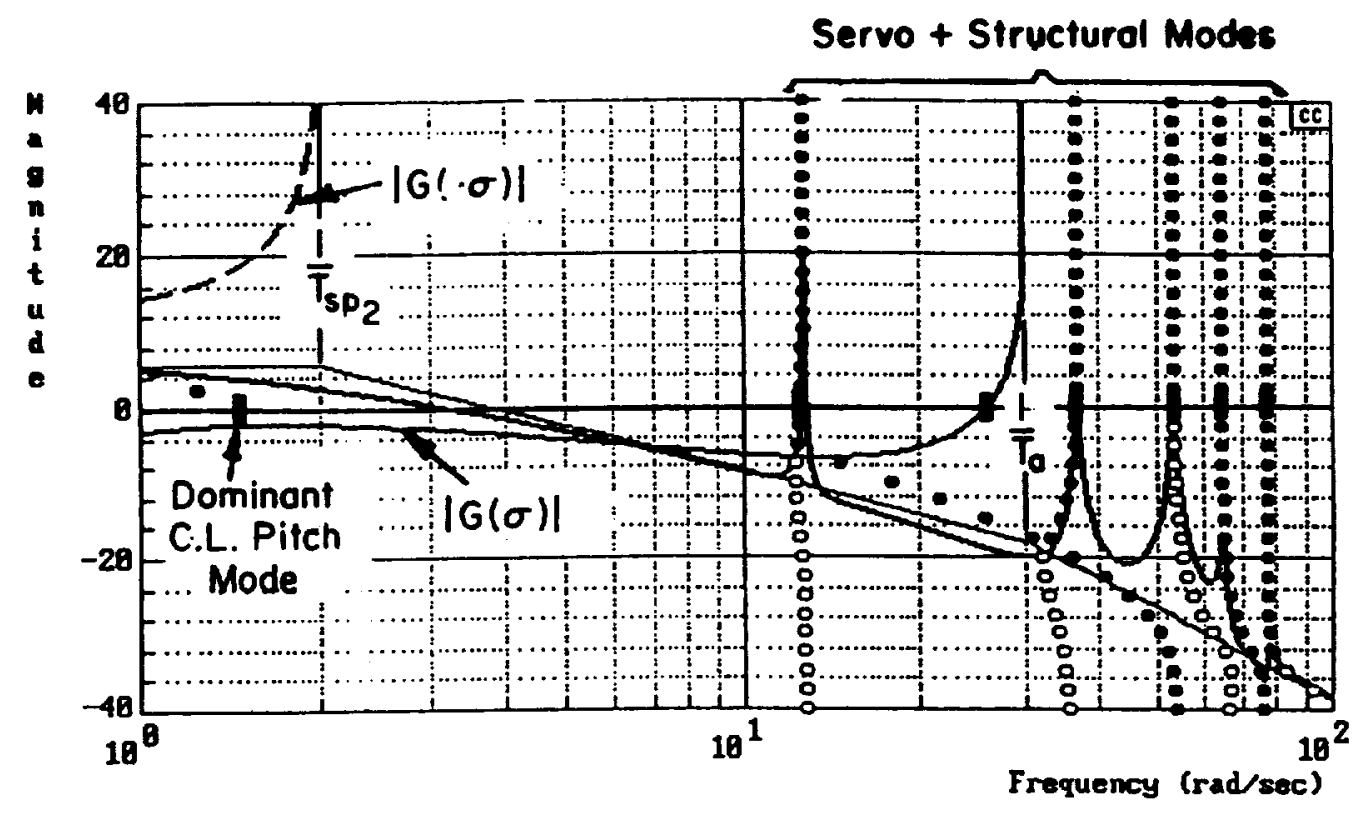

Bode Root Locus

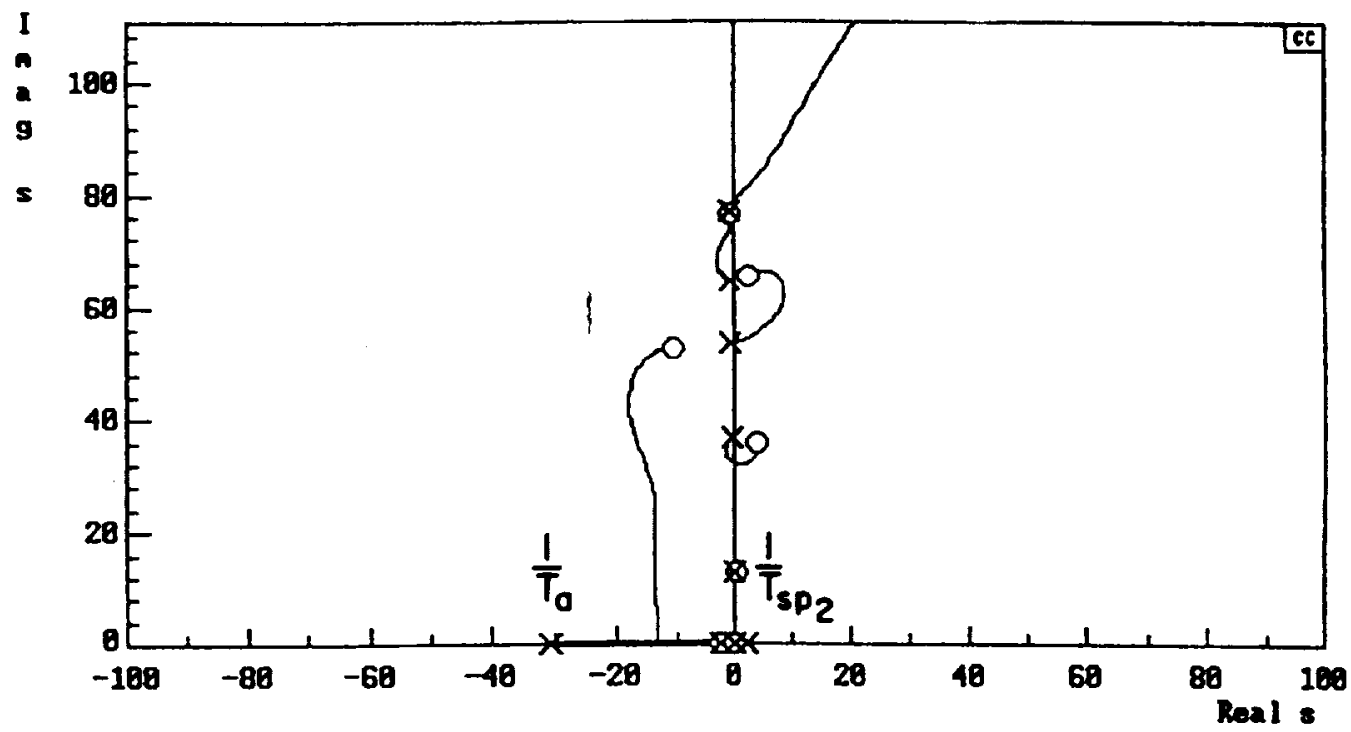

Conventional Root Locus

Figure 26. Baseline System Root Locus - Ascent Case 


\section{Section IV}

\section{CONVENTIONAL GAIN STABILIZED DESIGN}

As the primary reference for assessing the potential of the HPS design, a conventional gain stabilized flight control system design was developed for each of the two flight conditions (ascent at Mach 6 and descent at Mach 6). These systems consisted of the $2 \mathrm{rad} / \mathrm{sec}$ bandwidth design of Section III plus notch filters at the first structural mode and second order lags at somewhat higher frequencies. The filters for the two flight conditions are summarized in Table 4. The design for the ascent case will be addressed first.

\section{A. Ascent Case}

The open loop frequency response of the gain stabilized design is shown in Figure 27 and should be compared to that for the baseline (or the uncompensated) system of Figure 25. It can be seen that gain stabilization improves the gain margins such that 8 $\mathrm{dB}$ is exceeded for all modes. However, there is a significant reduction in phase margin which is an indication of the cost of gain stabilization.

Figure 28 presents the root locus which can be compared to the baseline in Figure 26. It can be seen that the lag filter has changed the pairings of poles and zeros for the loci.

Table 4. Filters Used in Gain Stabilized Designs

\begin{tabular}{|c||c|c|}
\hline Flight Condition & Notch Filter & Lag Filter \\
\hline \hline \multirow{2}{*}{ Ascent } & $\frac{[0.01,12.5]}{[0.35,12.5]}$ & $\frac{40.0^{2}}{[0.5,40.0]}$ \\
\hline Descent & $\frac{[0.0075,12.6]}{[0.30,12.6]}$ & $\frac{22.0^{2}}{[0.5,22.0]}$ \\
\hline
\end{tabular}

Notation: $[\zeta, \omega]=s^{2}+2 \zeta \omega s+\omega^{2}$ 

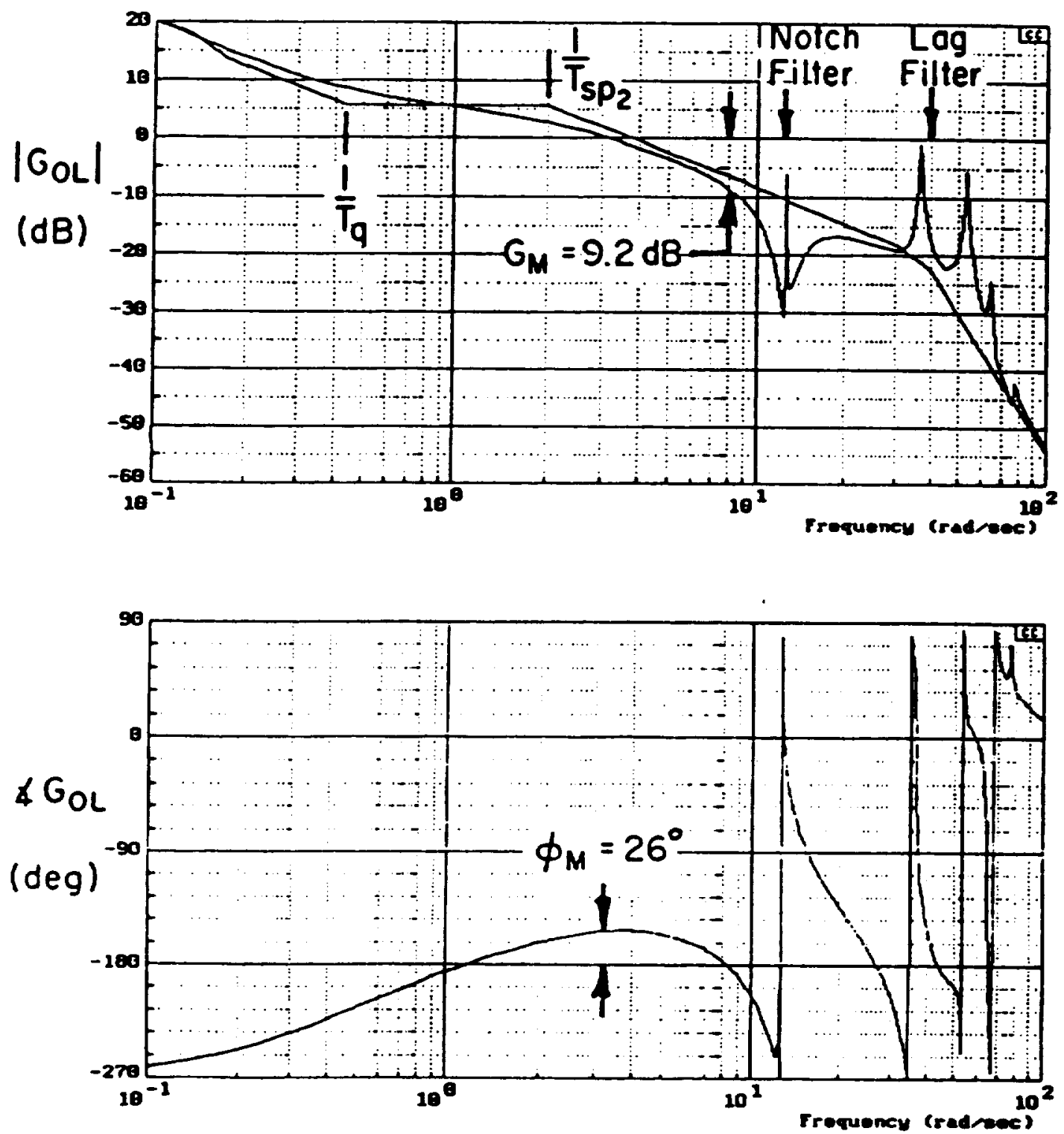

Figure 27. Gain Stabilized FCS Open Loop Frequency Response - Ascent Case 


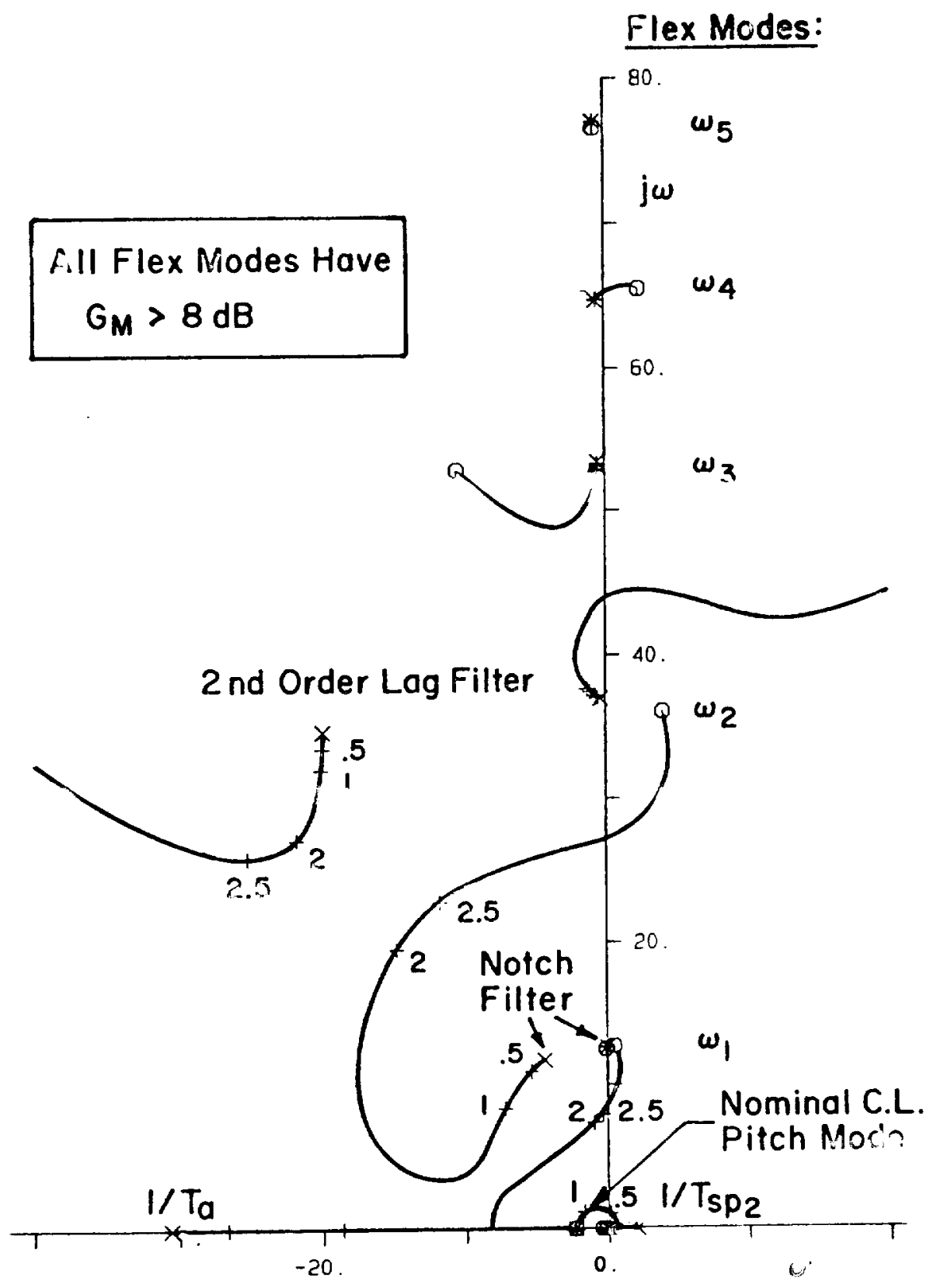

Fiquure 28. Gain Stabilized FCS Root Locus - Ascent Case 


\section{B. Descent Case}

In this section, the Mach 6, power off, descent case is examined. As in the ascent case, the pitch attitude bandwidth $\left(\omega_{\mathrm{B} W_{\theta}}\right)$ of $2 \mathrm{rad} / \mathrm{sec}$ is used. For a damping ratio $(\zeta)$ of 0.70 , using the airframe parameters in Table 1 and applying the Figure 23 algorithm and pole placement formula gives

$$
\begin{gathered}
\omega_{\mathrm{n}}=1.228 \mathrm{r} / \mathrm{s} \\
1 / \mathrm{T}_{\mathrm{q}}=0.32 \mathrm{r} / \mathrm{s} \\
\mathrm{K}_{\mathrm{q}}=1.176 \mathrm{r} / \mathrm{r} / \mathrm{s}
\end{gathered}
$$

The open loop frequency response of the "baseline" system (i.e., without the use of structural filters) is shown in Figure 29. The gain and phase margins are $4.22 \mathrm{~dB}$ and 35.14 degrees, respectively. As expected, the descent case margins are worse than the ascent case due to a higher unstable static margin.

The more conservative interpretation of attaining an $8 \mathrm{~dB}$ peak clearance for all structural modes is used in developing a gain stabilized design for the descent case. Figure 30 shows the open loop frequency response of this design. Although the $8 \mathrm{~dB}$ peak clearance for all structural modes was achieved, the gain margin was only improved to $4.88 \mathrm{~dB}$ (when compared to the "baseline" system).

Examining the two "baseline" system open loop frequency responses (Figure 25 and Figure 29) for both the ascent and descent cases, it appears that the structural filters used in the ascent case can possibly be applied to the descent case if the more conservative interpretation of attaining $8 \mathrm{~dB}$ peak clearance is not used as the design guideline. It can be shown that this gain stabilized design, without having to attain an $8 \mathrm{~dB}$ peak clearance for all structural modes, improves the gain margin to $6.61 \mathrm{~dB}$. 

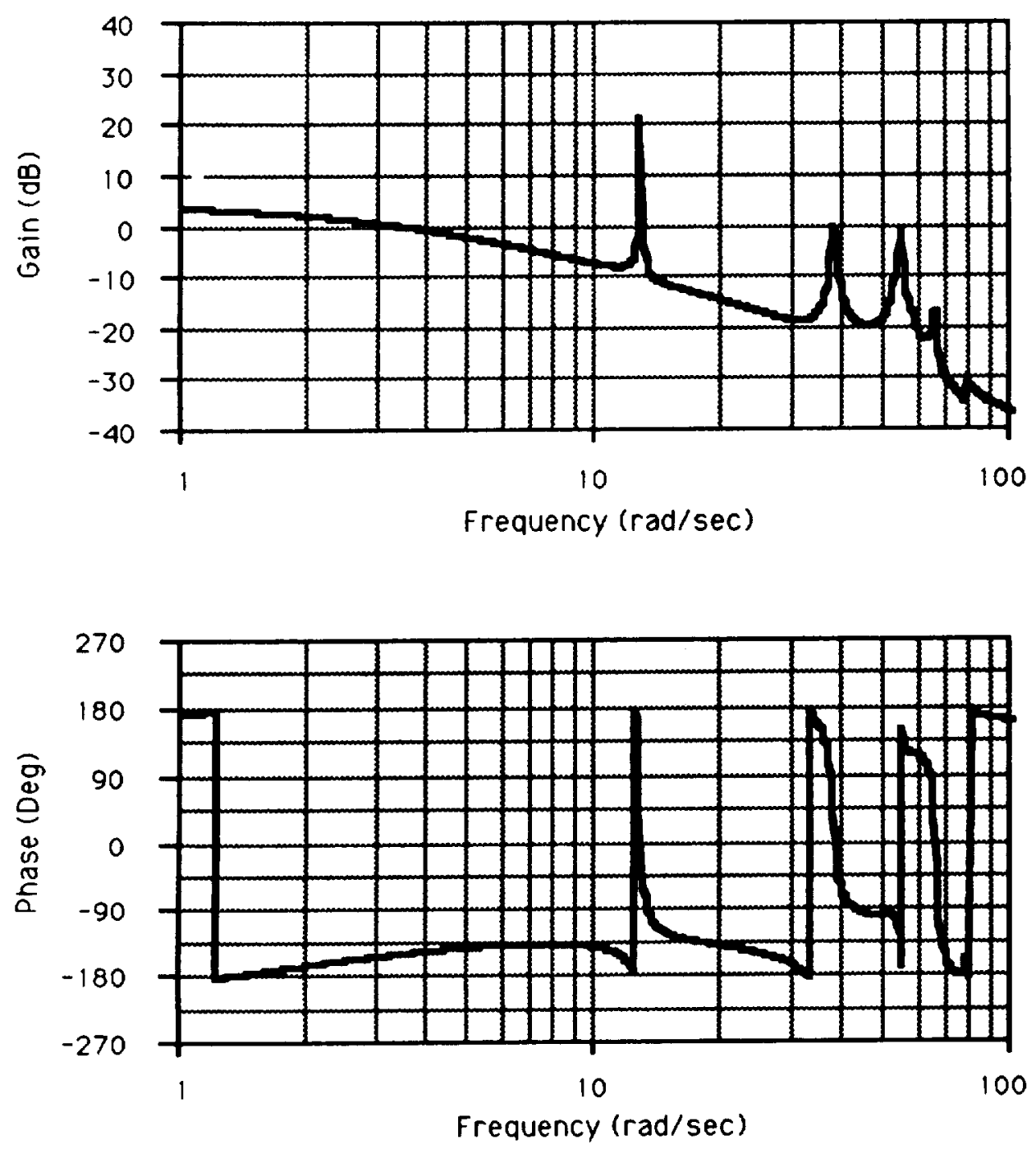

Figure 29. Baseline FCS Open Loop Frequency Response - Descent Case 

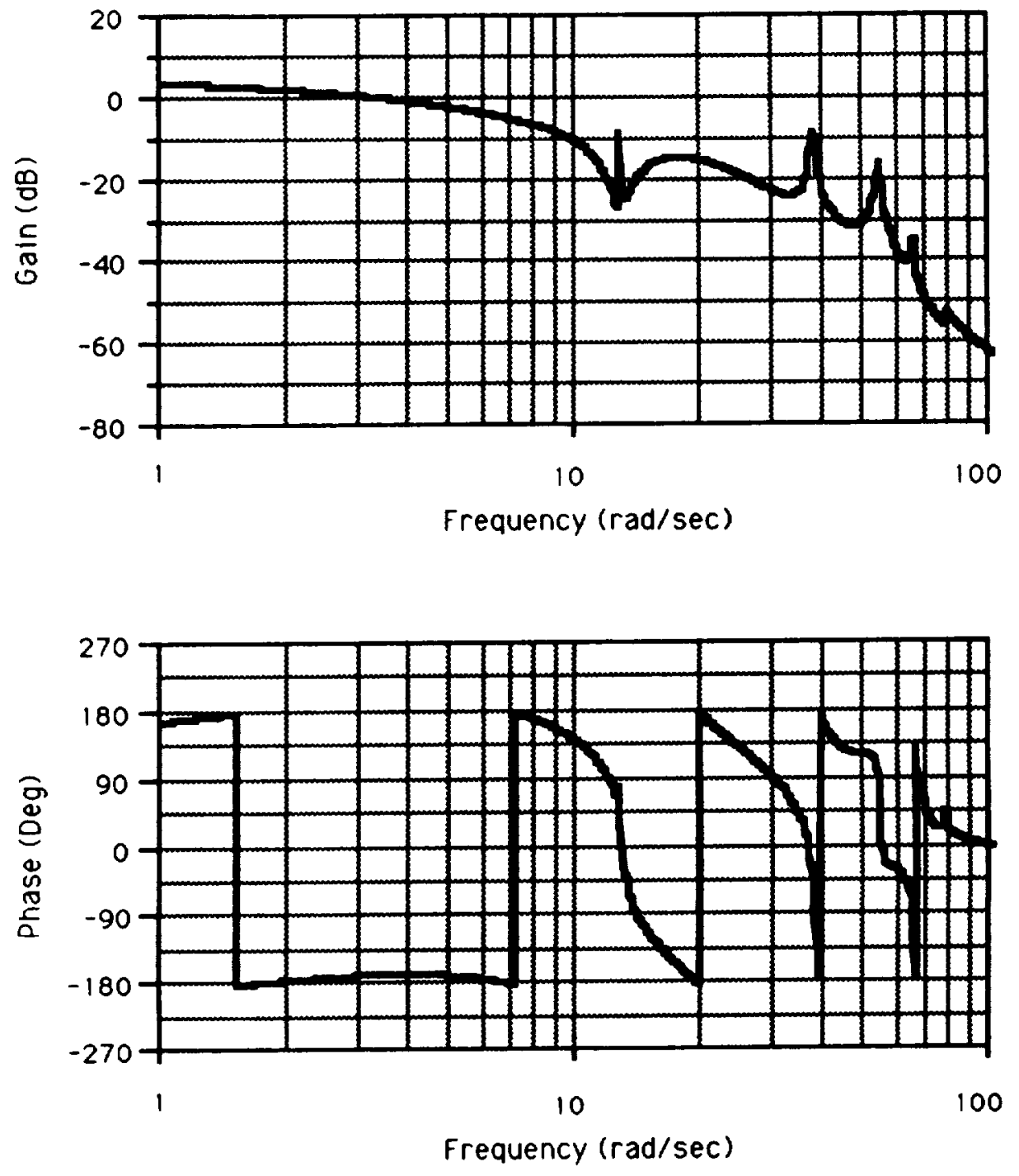

Figure 30. Gain Stabilized FCS Open Loop Frequency Response - Descent Case 


\section{Section V}

\section{HYBRID PHASE STABILIZED DESIGN}

This section addresses the primary focus of this contract -- the hybrid phase stabilized approach to flexible vehicle control. The details of HPS are best explained in the context of a detailed design example and this will be done next for the Mach 6, power on, ascent case. The basic rigid body FCS design is the $2 \mathrm{rad} / \mathrm{sec}$ superaugmented pitch loop developed in Section III-C.

\section{A. Ascent Case}

The key to phase stabilization is proper location of the sensors. For a pitch rate loop, the sensors are rate gyros. Figure 31 shows the location of the zero associated with the first structural pole for each of the four available sensor locations. The zero migration suggests that an effective zero could be positioned arbitrarily, within reason, by blending forward and aft pitch rate signals. It can be seen that the FS 84 and FS 1050 signal pair has the most potential.

Figure 32 outlines the signal blending concept. The inclusion of the filters (with unity low frequency gain) in each path provides additional flexibility in zero placement. As shown at the bottom of Figure 32, the signal blending problem can be manipulated into a root locus problem.

Figure 33 shows the zero placement root locus when the blending filters are pure gains. In this case the locus is a straight line between the two airframe zeros. To implement phase stabilization, we wish to position the zero directly below the structural pole and thus near the imaginary axis. As seen in Figure 33, the zero is on the imaginary axis when $\mathrm{K}_{\mathrm{a}} / \mathrm{K}_{\mathrm{f}}=0.92$.

The potential for improved closed loop damping is related to separation of the structural dipole. To some extent, separating the pole and zero will improve damping. As will be seen (and should be expected), the question is not as simple as maximizing dipole separation. In any case, dipole separation can be set by use of the blending filters. Figure 34 shows two filter sets from a more extensive survey. The filter poles are made 


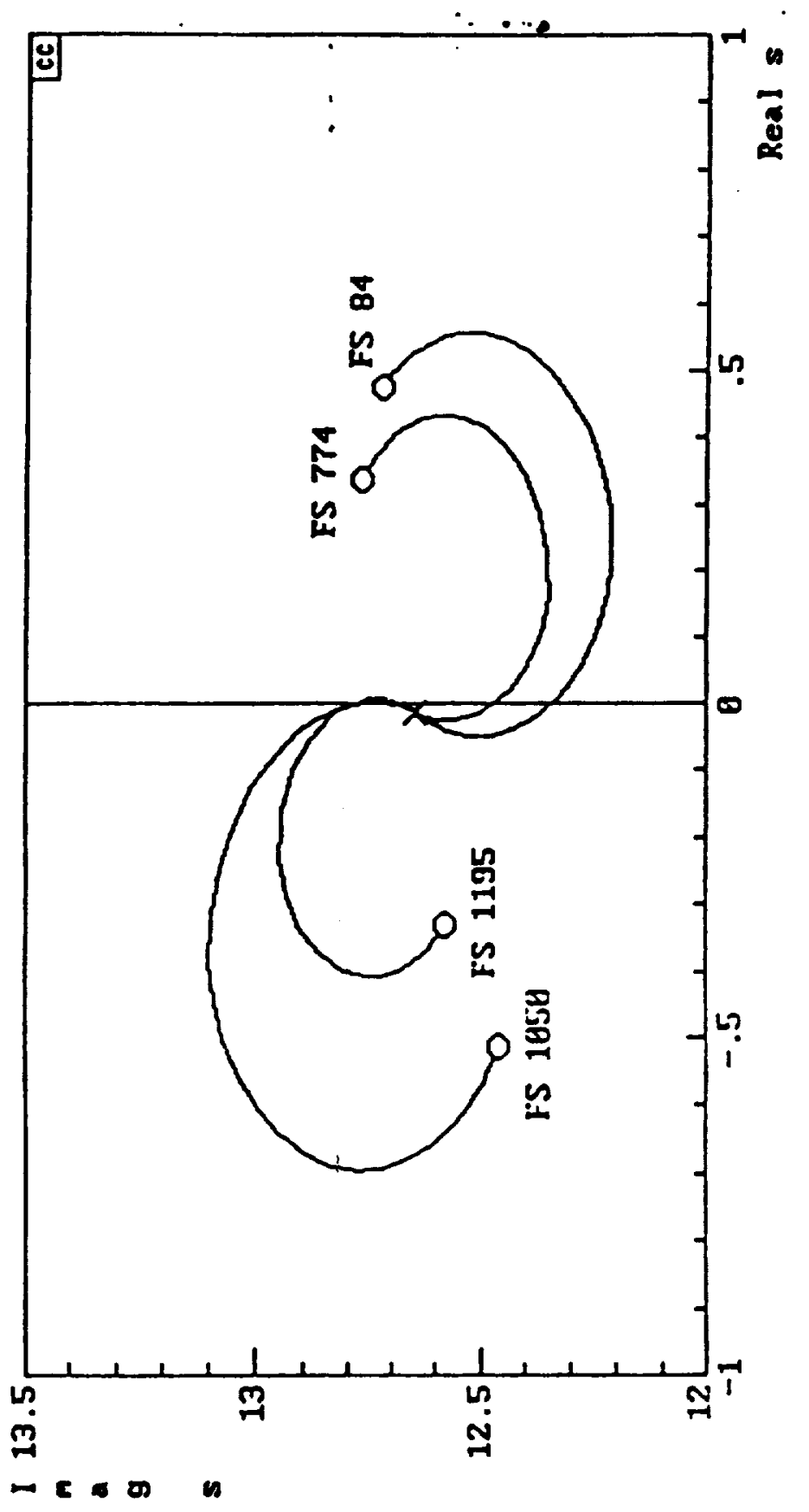

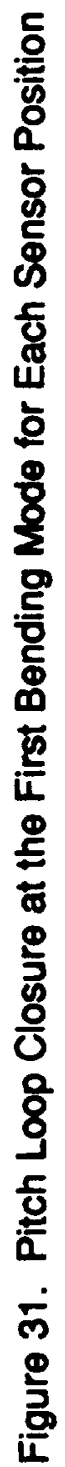



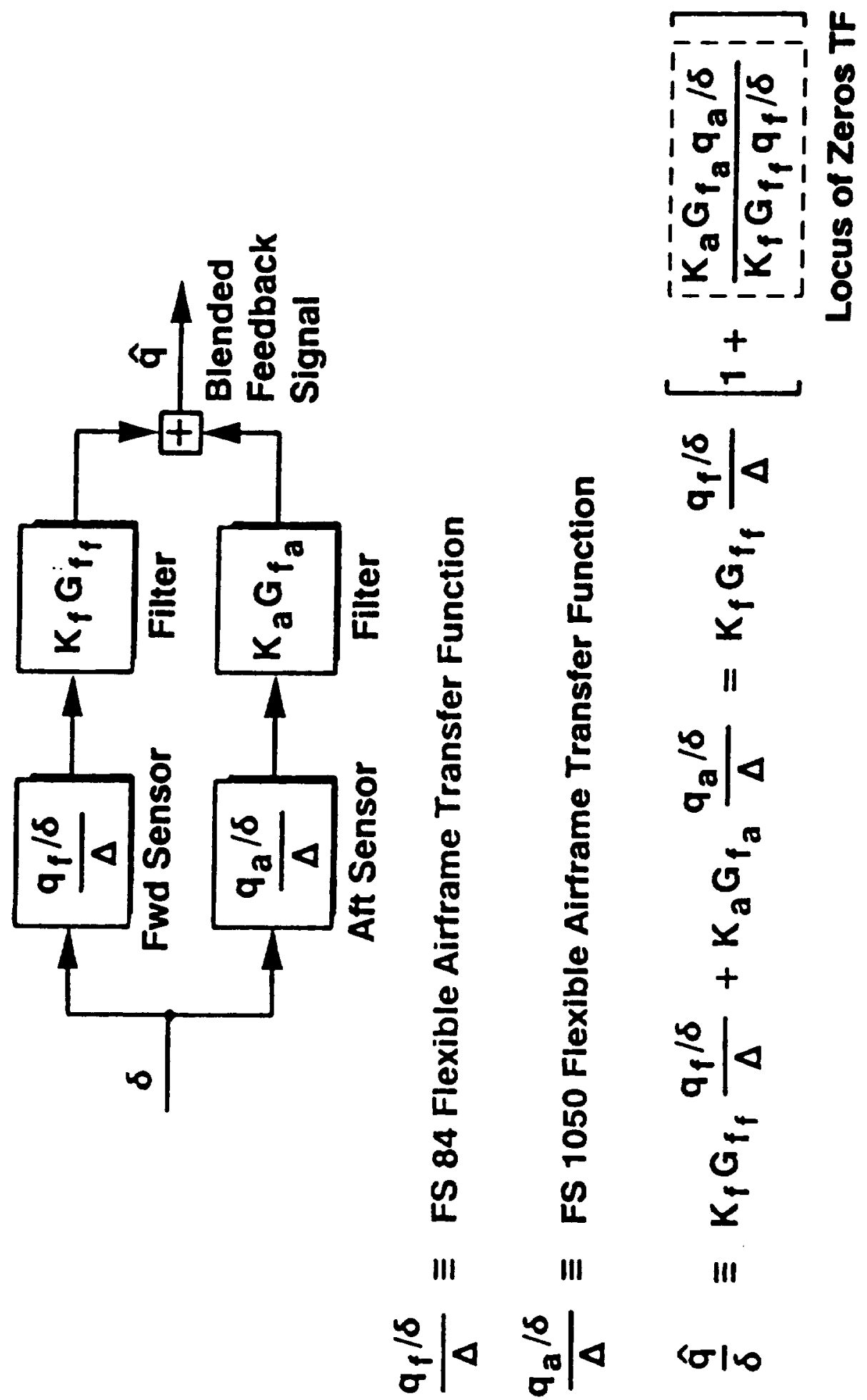

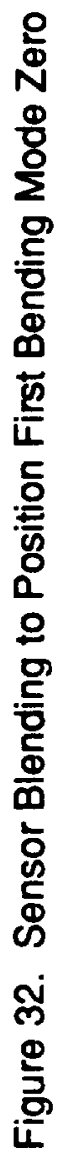




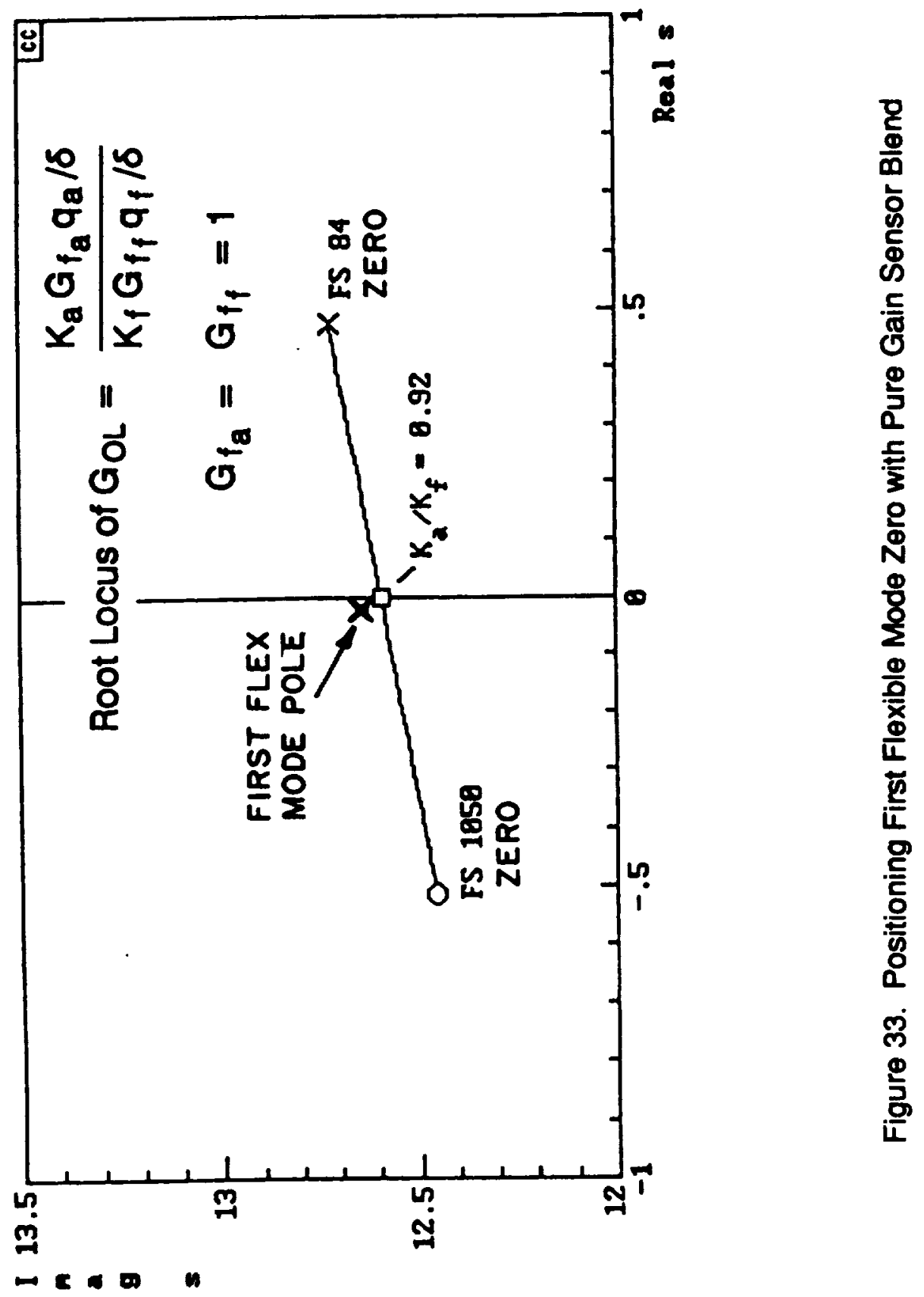



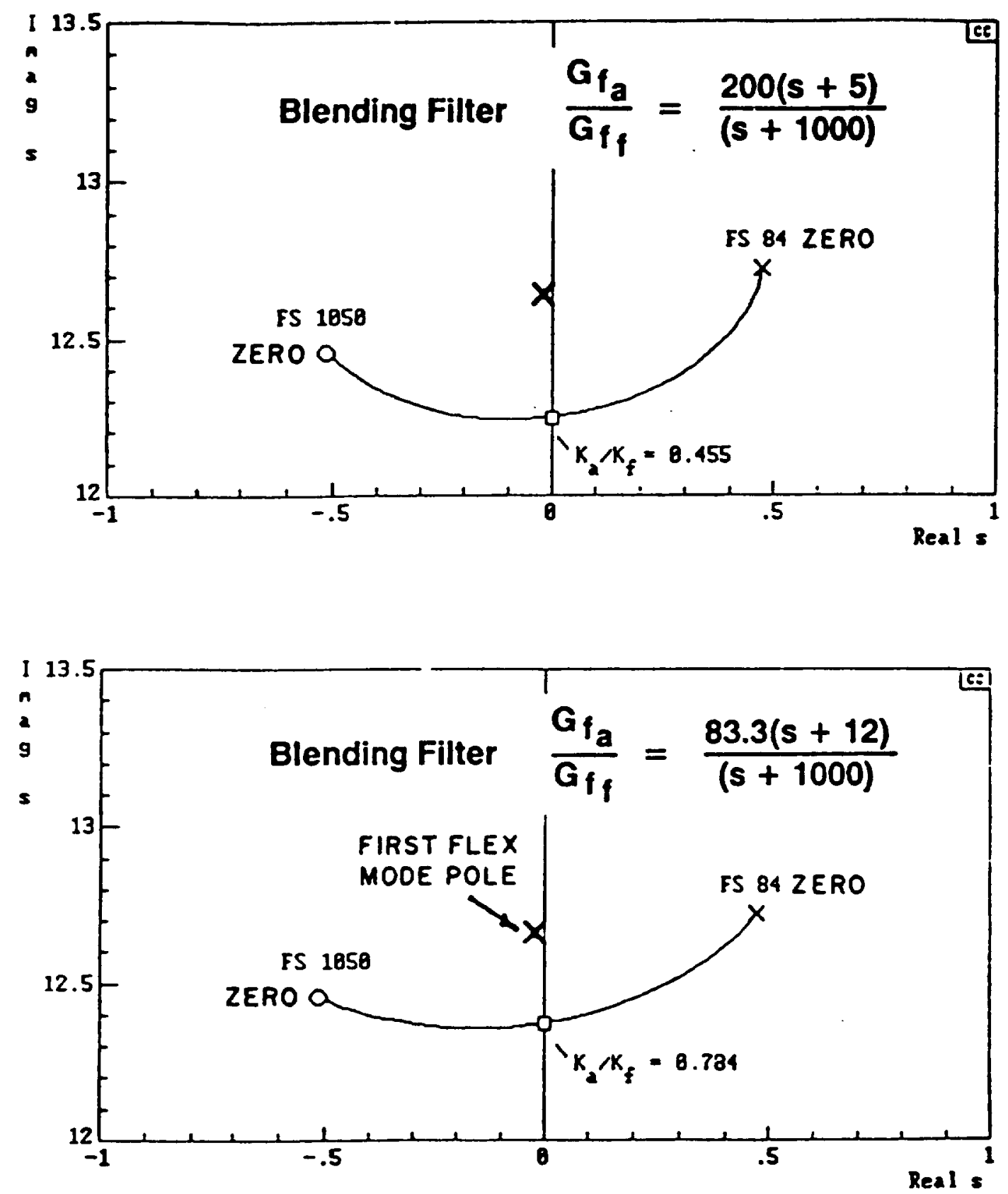

Figure 34. Positioning First Flexible Mode Zero with Filtered Sensor Blend 
equal in both paths (here they are set at $25 \mathrm{rad} / \mathrm{sec}$, but the design is not highly sensitive to this value). The zero in the forward path filter is fixed at a high value (1000 rad/ $\mathrm{sec})$, and thus the lead in the aft path filter becomes the primary filter design variable. The high frequency gains are always set to maintain the low frequency gains of both filters equal to unity.

Figure 34 shows that the dipole separation increases as the aft filter zero is reduced in frequency. However, there is a practical limit to how low this zero can be. Past a point it begins to distort the system in the crossover region. In particular, it begins to create a "shelf" in the open loop transfer function which tends to reduce the flex mode gain margins and thus would be at cross purposes with the phase stabilization effort. From the survey, the best blending filter position was selected as $12 \mathrm{rad} / \mathrm{sec}$.

The HPS superaugmented pitch loop is shown in Figure 35. The signal blending structure is used to phase stabilize the first structural mode and $\mathrm{G}_{\mathrm{LAG}}$ is the first order lag (with unity low frequency gain) used to gain stabilize the higher frequency modes. Because the lag does not have to handle the lowest frequency structural mode, it can be placed at an unconventionally high frequency. This is the key to achieving minimum effective time delay by treating the first structural mode with HPS.

A final element required in the HPS system is the block just before the final lag (Figure 35). This gain function insures that the $q$ loop crossover region is unchanged by the signal blending.

Figure 36 shows a first look at the HPS q loop closure without the lag (i.e. with $\mathrm{G}_{\mathrm{LAG}}=1$ ). When compared to the baseline (or the uncompensated) system survey in Figure 26, it can be seen that the crossover region is essentially unchanged. The next observation is that, with the blended feedback signal unlike the baseline system, the first flex mode is stable for all gains. The conventional root locus shows that, as advertized, the locus bulges into the left half plane. However, the closed loop pole for the nominal closure does not fully exploit the potential for improved damping. Moreover, the gain cannot be increased to further increase structural damping without adversely affecting the dominant pitch mode dynamics. It may be that decreasing the separation of the first structural dipole would lead to increased damping, but further optimization of this was not possible in this study. The final observation from Figure 36 is that the third structural mode is now the primary problem; this will set the requirement for $\mathrm{G}_{\mathrm{LAG}}$. 


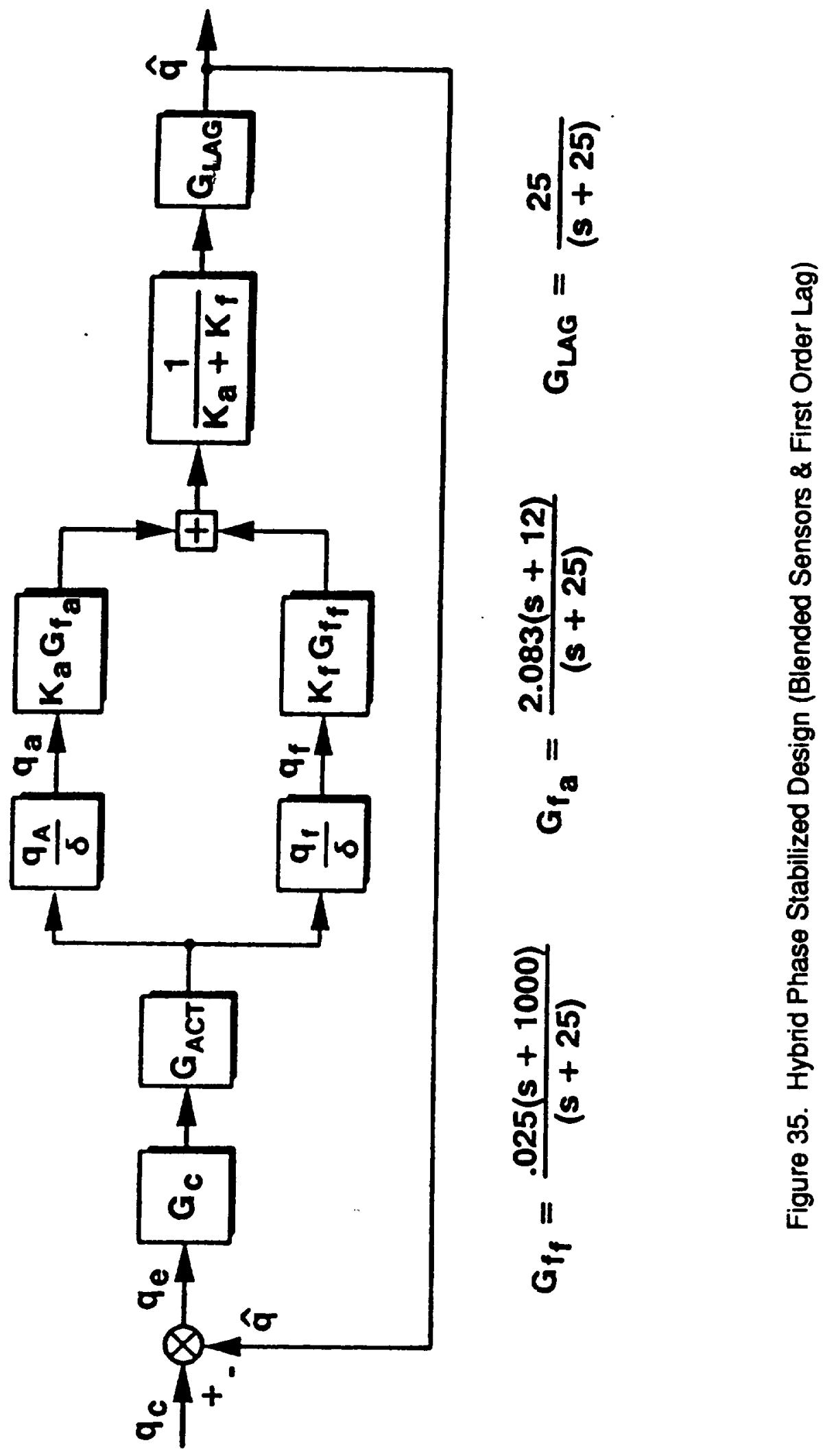




\section{Servo, Blending Filters \& Structural Modes}

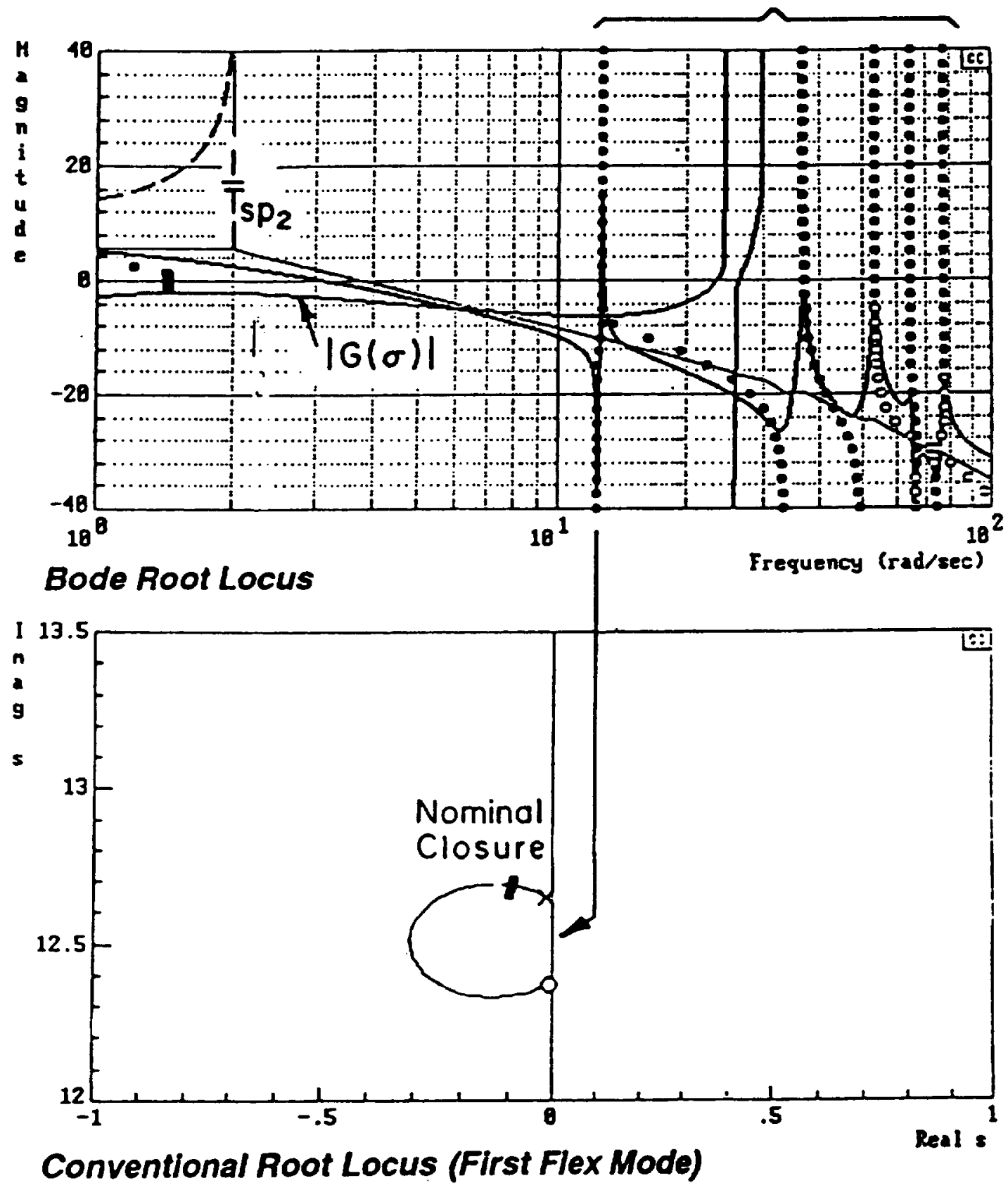

Figure 36. Pitch Loop Closure with Sensor Blend (12 rad/sec Blending Filter) 
A survey of lags was made and it was found that a $25 \mathrm{rad} / \mathrm{sec}$ lag was the highest value with acceptable gain margin on the critical structural mode (in this case, the second mode). Figure 37 shows the open loop frequency response of the final HPS design. It should be noted that not only does the first (phase stabilized) flex dipole show a positive phase "blip", but next two do as well, so that a "sawtooth characteristic" has been achieved. The magnitude peaks have been lowered on all flex modes except the first. The significance of this last point will be addressed in Section VI-B.

Figure 38 shows the system survey for the final HPS design for the ascent case. When compared to Figure 36 , it can be seen that the second structural mode now has the minimum stability margin but is acceptable.

\section{B. Descent Case}

Without repeating the detailed description of the hybrid phase stabilized design procedure for the descent case, it was found that the blending filters and gains (i.e., $G_{f a}$, $\mathrm{G}_{\mathrm{ff}}, \mathrm{K}_{\mathrm{a}}$ and $\mathrm{K}_{\mathrm{f}}$ ) developed for the ascent case can also be used here. However, the break frequency of $G_{L A G}$ must be reduced to at least $22 \mathrm{rad} / \mathrm{sec}$ in order to meet the $8 \mathrm{~dB}$ gain margin requirement for all structural modes. The open loop frequency response for this phase stabilized design is shown in Figure 39. It should be kept in mind that, in some flight conditions, the blending filters might need to be re-tuned.

The pitch rate response to a unit step pitch rate command is shown in Figure $\mathbf{4 0 .}$ Responses of the gain stabilized and baseline designs are also included for comparison. The time response behavior will be discussed in Section VI-B. 


\section{Servo + Structural Modes}
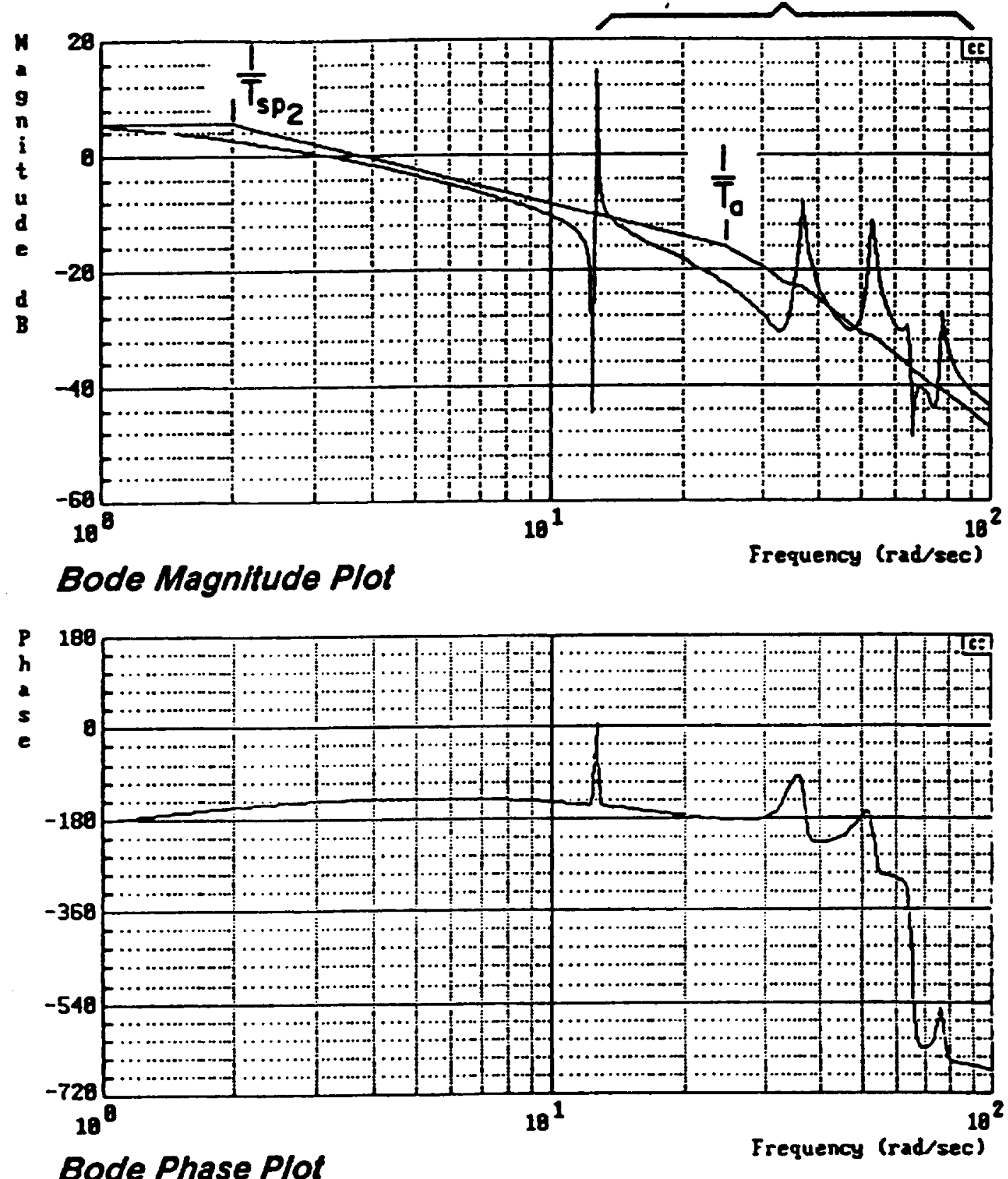

Figure 37. Phase Stabilized Design Open Loop Transfer Function (Blended Sensor \& First Order Lag) - Ascent Case 

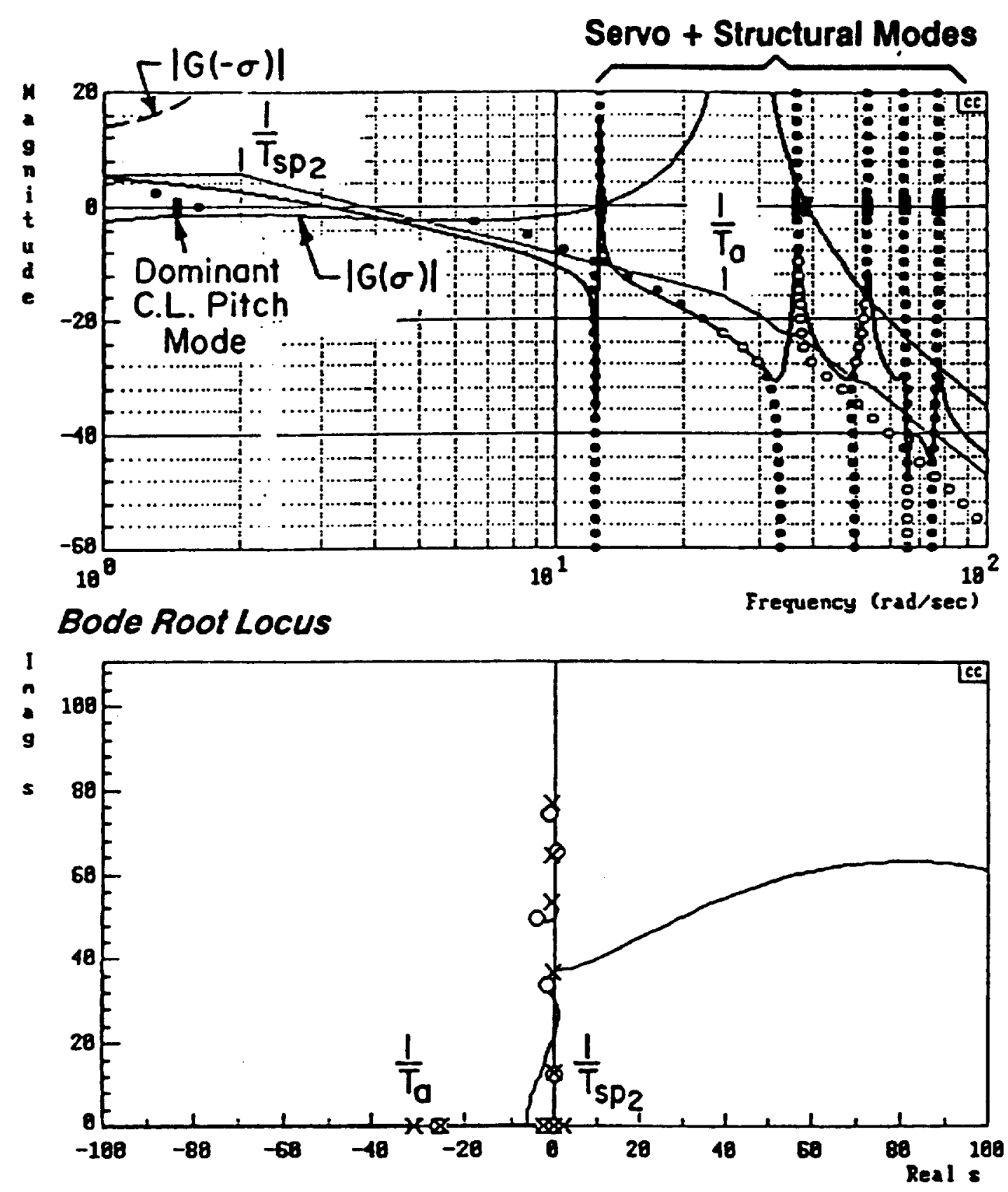

Conventional Root Locus

Figure 38. Phase Stabilized Design Closure (Blended Sensor \& First Order Lag) - Ascent Case 

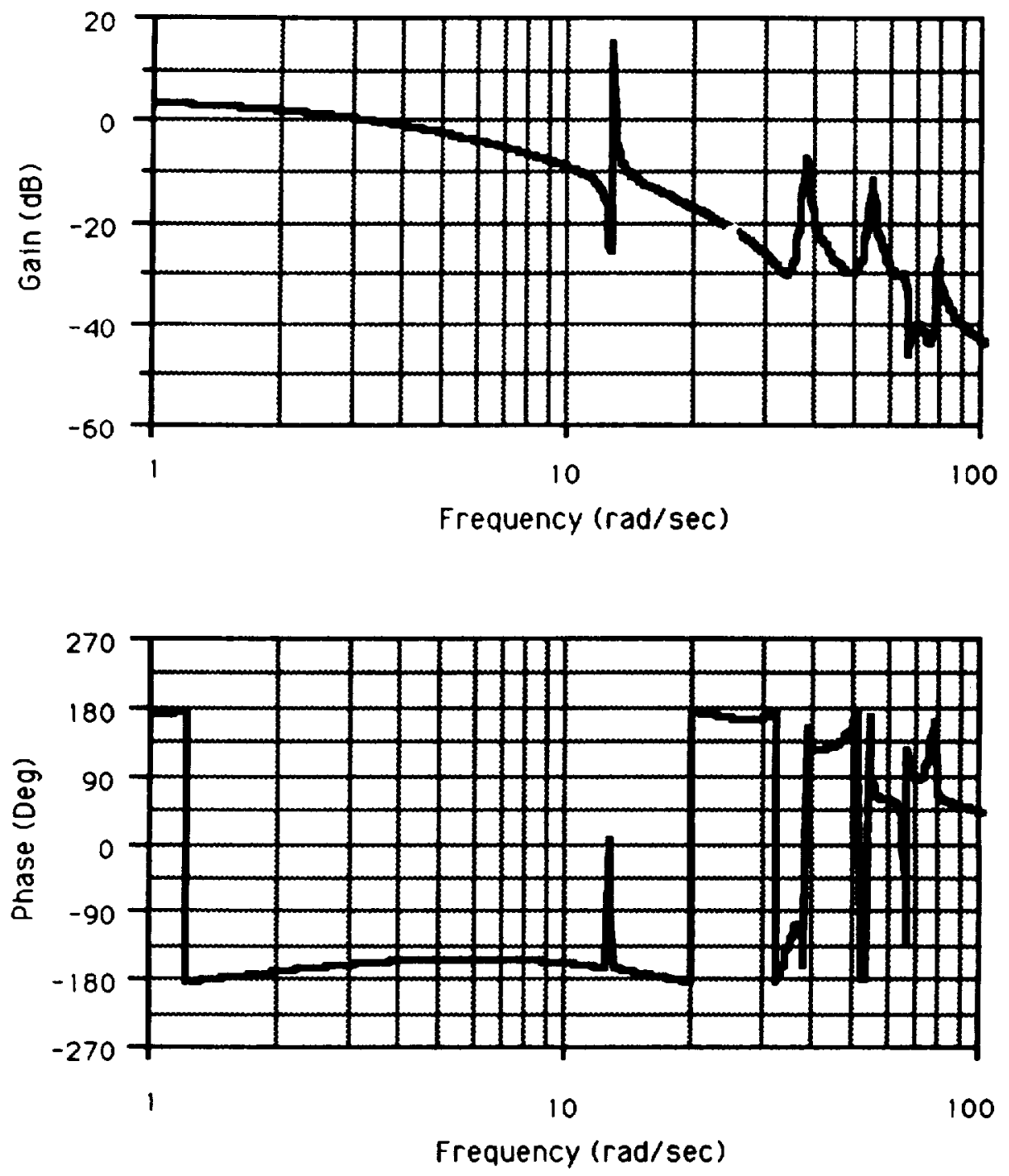

Figure 39. Phase Stabilized Design FCS Open Loop Frequency Response - Descent Case 


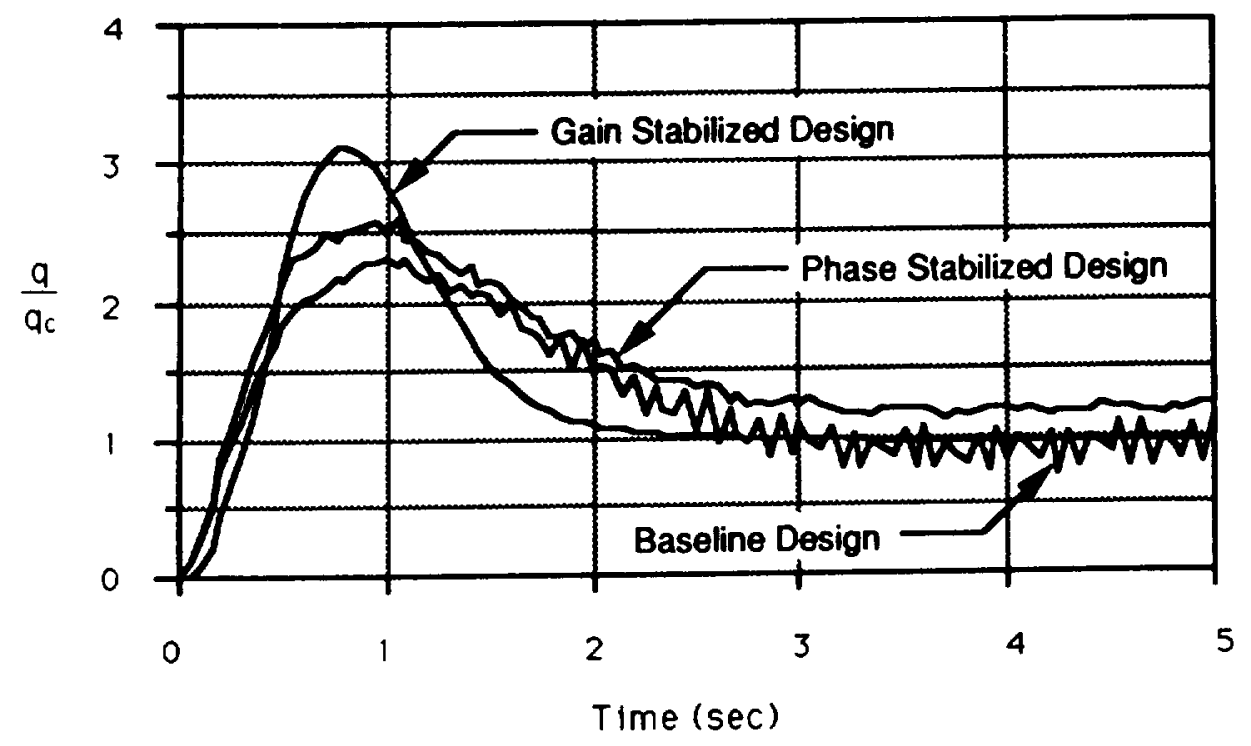

Figure 40. Pitch Rate Response to a Unit Step Pitch Rate Command - Descent Case 
This page is intentionally left blank. 


\section{Section VI}

\section{DESIGN COMPARISONS}

\section{A. Stability Metrics}

\section{Ascent Case}

Since a primary motivation for HPS is reduction of the effective time delay penalty associated with conventional gain stabilization, this issue will be examined first under the heading of general stability metrics. Table 5 compares four stability metrics for the ascent case. The open loop metrics are gain, phase and time delay margin $\left(\tau_{M}\right)$. The gain margin is that for the phase crossover closest to the first structural mode. The time delay margin is the additional time delay in the loop that would drive the phase margin to zero. The closed loop equivalent system time delay $\left(\tau_{e}\right)$ is computed from a low frequency approximation of the closed loop $\mathrm{q}$ to $\mathrm{q}_{\mathrm{c}}$ transfer function above the dominant pitch mode but below the first bending mode.

The Table 5 metrics illustrate that HPS reduces the equivalent system time delay compared to conventional gain stabilization. While HPS has a time delay cost beyond the baseline (no structural mode treatment), this increment is considerably less than the gain stabilization increment.

Table 5. Stability Metric Comparison - Ascent Case

\begin{tabular}{|c||c|c|c||c|}
\hline DESIGN & $G_{M}(\mathrm{~dB})$ & $\phi_{M}(\mathrm{Deg})$ & $\tau_{M}(\mathrm{sec})$ & $\tau_{\theta}(\mathrm{sec})$ \\
\hline \hline Baseline & 5.13 & 41.8 & 0.223 & 0.051 \\
\hline Gain Stabilized & 9.20 & 26.2 & 0.142 & 0.218 \\
\hline Phase Stabilized & 20.26 & 35.3 & 0.192 & 0.077 \\
\hline
\end{tabular}


For reference in the next section, it should be noted that the HPS gain margin $(20.3 \mathrm{~dB})$ is much higher than either the baseline or the gain stabilized design. More importantly, it far exceeds the $8 \mathrm{~dB}$ ML-spec requirement. However, comparison of Figures 37 and 27 show that the magnitude peak at the first structural mode is well above $0 \mathrm{~dB}$ and much higher than that of the gain stabilized design.

This observation touches on an important question about the present MIL-specs. A "strict constructionist" view says that the HPS design satisfies the gain margin requirement. However, in practice the spec is often interpreted as requiring an $8 \mathrm{~dB}$ of "peak clearance" below the $0 \mathrm{~dB}$ line, which is in general a much more stringent requirement. The more conservative peak clearance interpretation has often been acceptable for design guidance when gain stabilization was assumed, bandwidth requirements were low and airframe static margin was high. But for HSVs, there are strong and conflicting design pressures toward highly unstable static margins combined with low structural mode frequencies that force us to reexamine the FCS design approach and the stability specifications. The latter point will be considered further in the next section.

\section{Descent Case}

The gain and phase margin and the closed loop equivalent system time delays are tabulated in Table 6. As expected, the results follow a similar trend as the ascent case. The gain margin for the phase stabilized design far exceeds the MIL-spec requirement of 8 dB.

Table 6. Stability Metric Comparison - Descent Case

\begin{tabular}{|c||c|c|c|}
\hline DESIGN & $G_{M}(\mathrm{~dB})$ & $\phi_{M}(\mathrm{D} \Theta \mathrm{g})$ & $\tau_{\theta}(\mathrm{sec})$ \\
\hline Baseline & 4.22 & 35.14 & 0.0551 \\
\hline Gain Stabilized & 4.88 & 15.03 & 0.2467 \\
\hline Phase Stabilized & 16.44 & 27.04 & 0.0777 \\
\hline
\end{tabular}


It should be repeated here that the gain stabilized design goal for the descent case was to make sure that all structural mode peaks stay below $-8 \mathrm{~dB}$. Figure 30 shows that the gain margin closest to the first structural mode occurs at $7 \mathrm{rad} / \mathrm{sec}$ which is below the first structural mode. The FCS spec requires a gain margin of at least $+6 \mathrm{~dB}$. Clearly the MIL-spec requirement was not met. For vehicles with very low structural mode frequencies, HPS may be the only technique of choice if the MIL-spec requirement is to be met.

\section{B. Residual Response}

The reduction in effective time delay with HPS was expected and in itself a concept worth pursuing. However, as noted previously, the question of uncommanded high frequency response (residual response) is also critical but more difficult to deal with. This is closely connected to the interpretation of the gain margin requirements noted in the previous section. The perspective for HSVs is that this class of aircraft is likely to present a considerable challenge to meeting existing stability requirements. All possible solutions and requirements will probably have to be carefully reexamined. In particular, the gain margin specs may have to be reexamined and possibly relaxed. To do this rationally, metrics for quantifying residual response are required.

As a first step, the pitch rate, elevator position and normal acceleration responses to a unit step pitch rate command for the two designs are compared in Figures 41, 42 and 43 respectively. The $q$ and $n_{z}$ responses are for the pilot's station (actually FS 84). However, the $n_{z}$ signal is an estimate since an actual signal was not available. In all cases, the gain stabilized design shows very little residual response after several seconds. The HPS design's residual pitch response is probably no better or worse than the baseline and may not be much worse than the gain stabilized design. In the elevator response, the gain stabilized and HPS residual response are comparable and minimal compared to the baseline.

The load factor comparison is probably of most importance. In the gain stabilized design, the residual response essentially vanishes after about four seconds, whereas the HPS residual response is comparable to the baseline response in magnitude but with a somewhat different frequency content. The HPS residual response appears to be dominated by the second structural mode with some contribution from the first. 


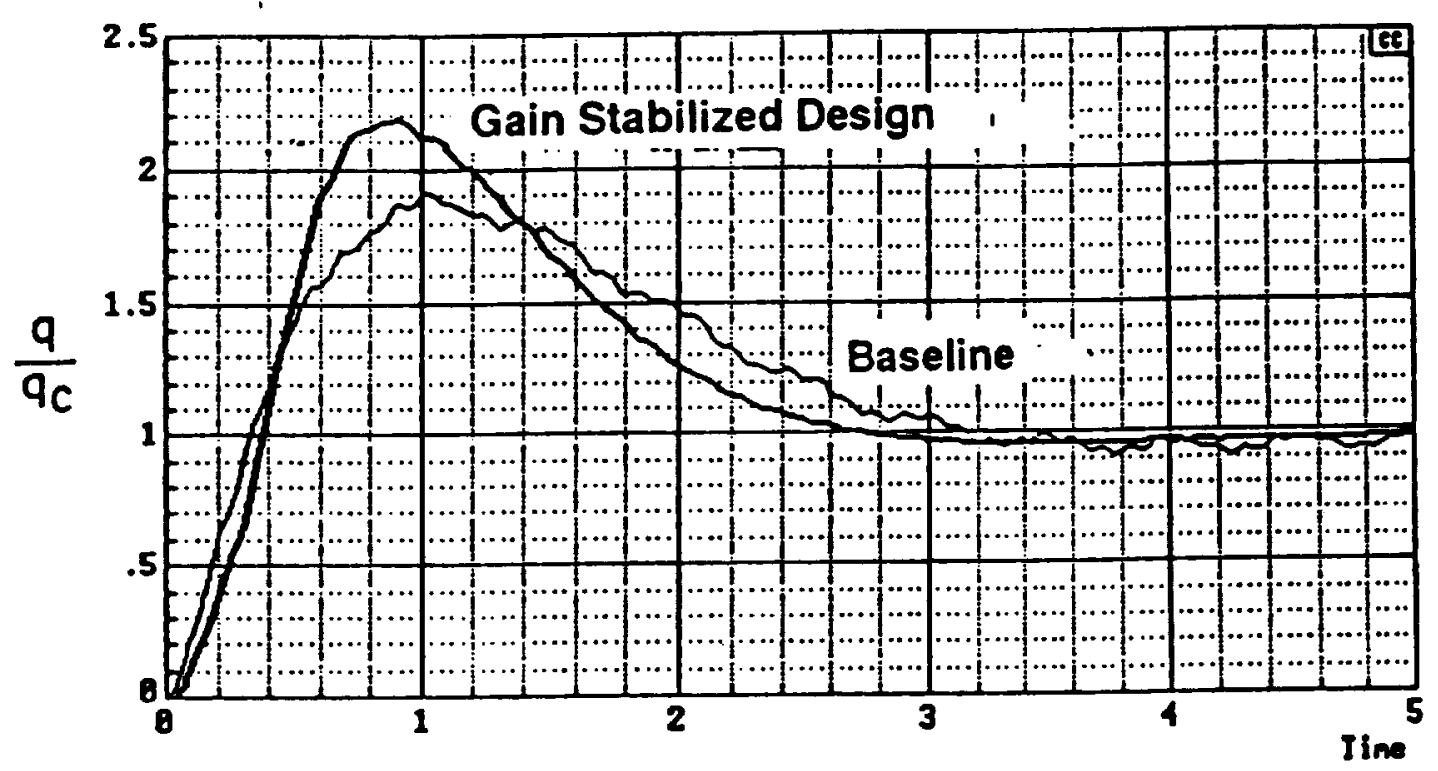

Measured at Pilot Location (FS=84)

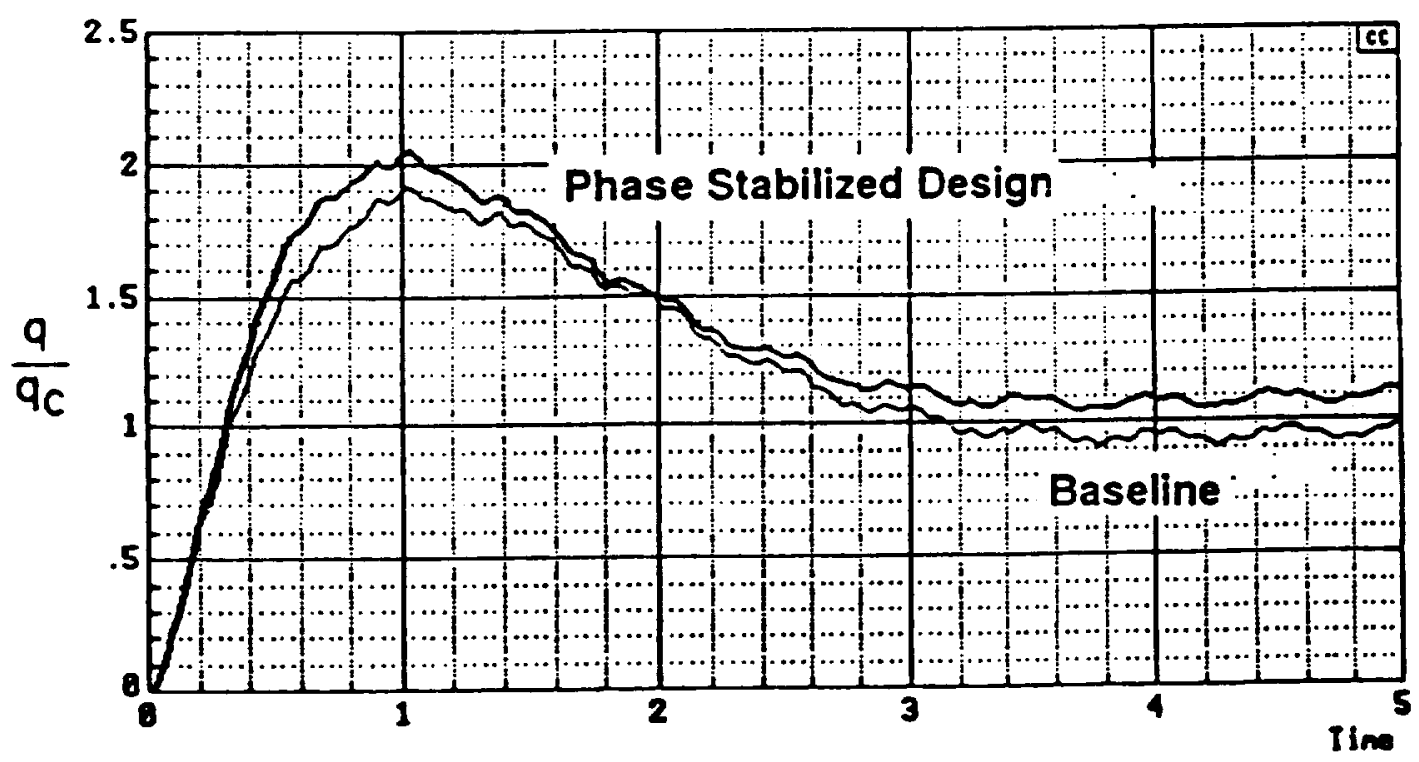

Figure 41. Response Comparison of Systems, Pitch Rate Response to Unit Pitch Rate Command - Ascent Case 

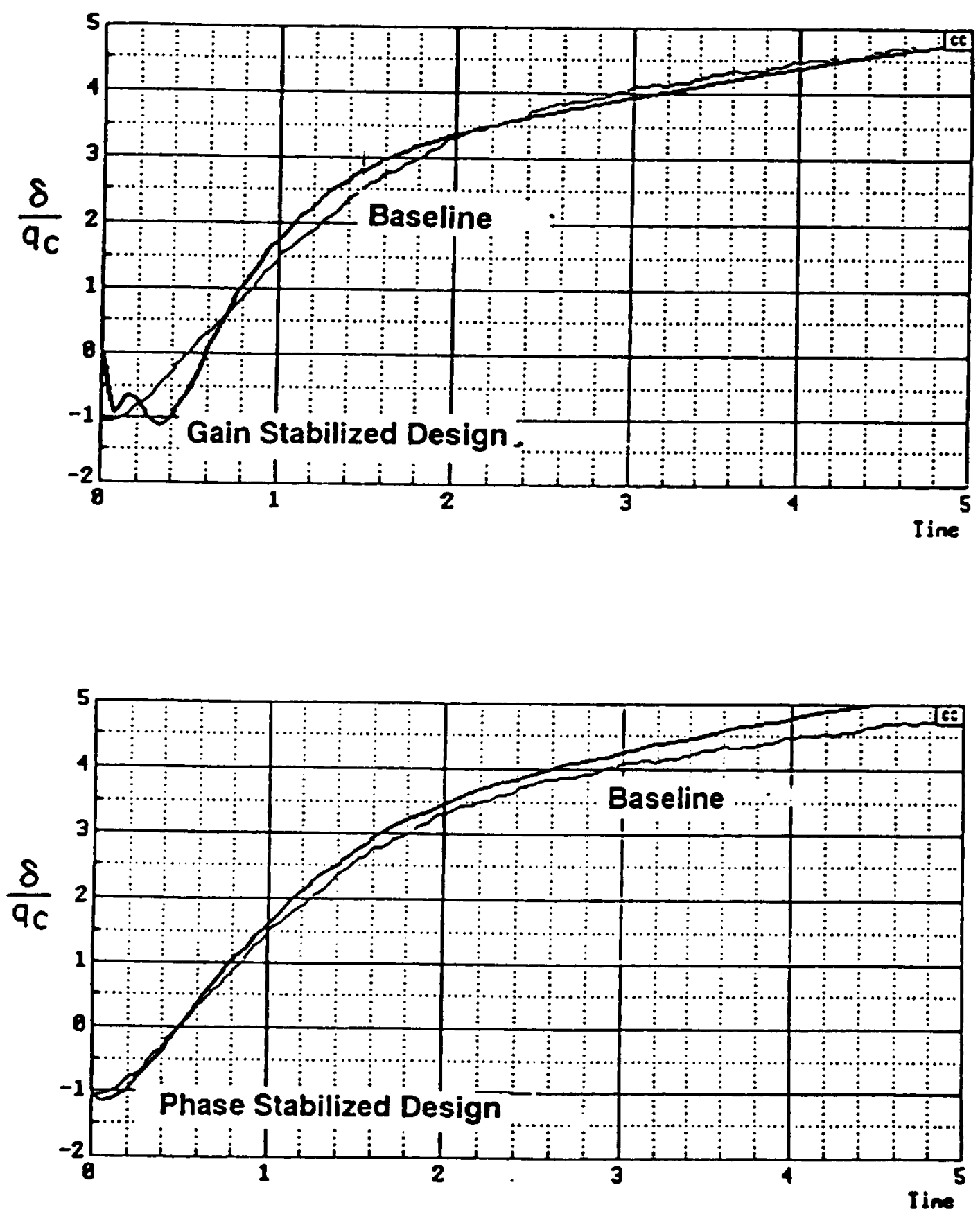

Figure 42. Response Comparison of Systems, Elevator Response to Unit Pitch Rate Command - Ascent Case 


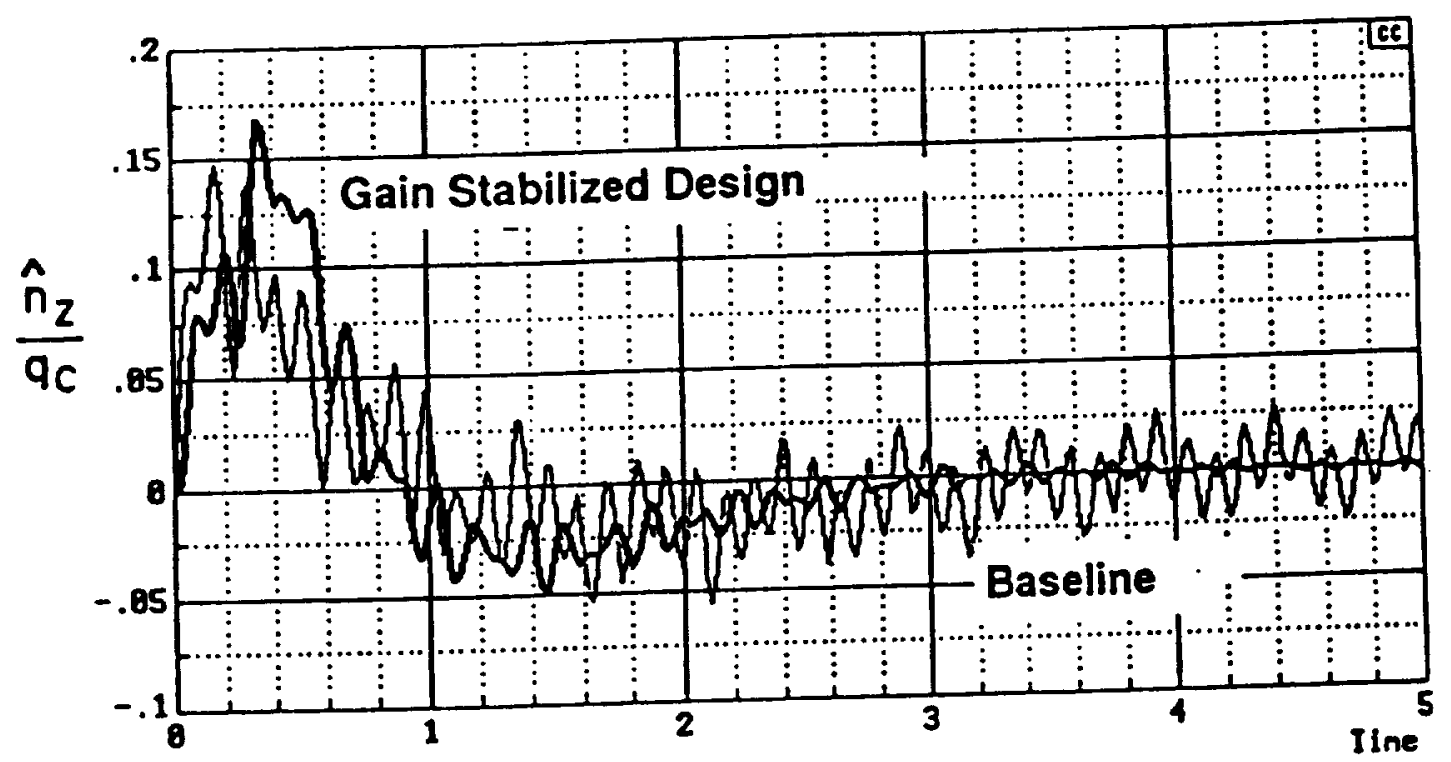

Measured at Pilot Location (FS=84)

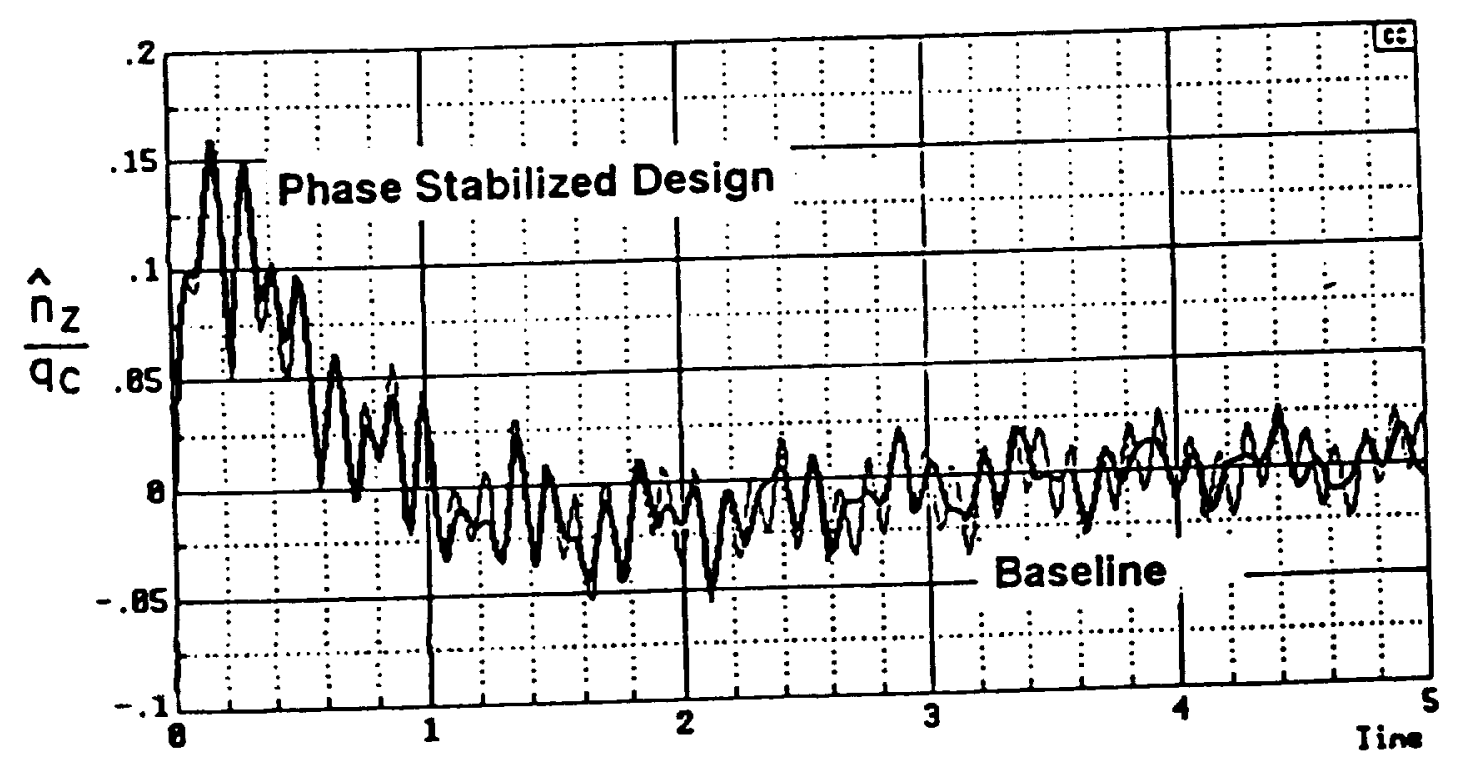

Figure 43. Response Comparison of Systems, Estimated Normal Acceleration Response to Unit Pitch Rate Command - Ascent Case 
The real problem is establishing the significance of the HPS residual response, a question that could only be briefly explored in this study. Appropriate residual response metrics are needed, and while some existing specifications can be brought to bear, this area requires considerable work and validation before the MIL-spec requirements can be relaxed to allow HPS as a possible solution for HSVs.

As a first step, an exploratory metric was briefly examined. This is diagrammed in Figure 44. The first three blocks represent an abstraction of normal acceleration response at the pilot's station. The input is a generic stochastic signal which has characteristics comparable to either Dryden turbulence or pilot remnant. The closed loop pitch transfer function reflects the FCS design to be rated. The "s/32" block provides an empirically based estimate of the pilot station load factor. As noted previously, the available structural model did not provide normal acceleration data, but when this is available, the representation can be improved. The final element, the structural response weighting filter, provides a means of emphasizing the residual structural response that is the focus of the metric. The weighted rms normal acceleration is the primary metric.

Table 7 compares the residual response metric of the two designs normalized by the baseline (or the uncompensated) value for the ascent case. According to the tentative metric, the gain stabilized design has the greater attenuation, but apparently only slightly more than the phase stabilized design. This result differs somewhat from the subjective impression of the Figure 43 comparison. Its validity could not be further assessed in this study. However, it does indicate a direction for further development as well as potential difficulties in validating such metrics.

Table 7. Residual Response Metric Comparison - Ascent Case

\begin{tabular}{|c||c|c|}
\hline DESIGN & $\left(\frac{\sigma}{\sigma_{\text {BASE }}}\right)_{\mathrm{q}}$ & $\left(\frac{\sigma}{\sigma_{\text {BASE }}}\right)_{\hat{n}_{2}}$ \\
\hline \hline Baseline & 1.00 & 1.00 \\
\hline Gain Stabilized & 1.17 & 0.673 \\
\hline Phase Stabilized & 0.916 & 0.698 \\
\hline
\end{tabular}




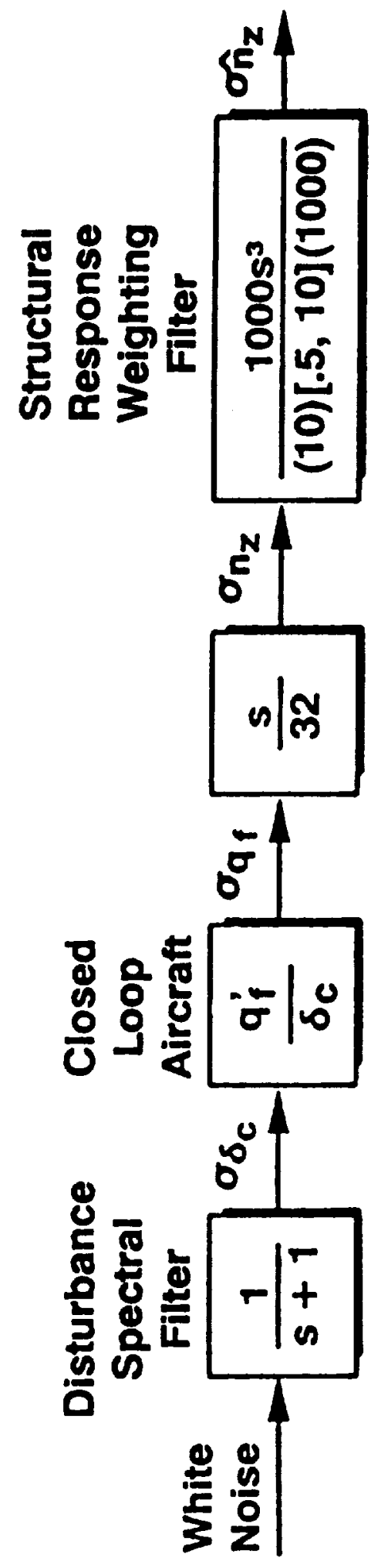

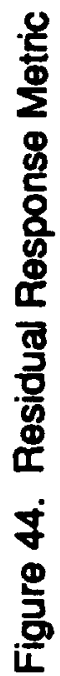




\section{Section VII}

\section{SUMMARY, CONCLUSIONS AND RECOMMENDATIONS}

\section{A. Summary}

In this contract, a preliminary examination of Hybrid Phase Stabilization (HPS) for application to HSVs has been made. Major activities included:

- Development of a linear, flexible model of an HSV operating at hypersonic speeds.

- Development of example HPS designs for Mach 6 ascent and descent.

- Comparison of the HPS designs to conventional gain stabilized designs at two flight conditions.

\section{B. Conclusions}

- The HPS concept does significantly reduce the effective time delay.

- The HPS design, as presently developed, shows greater residual response than a conventional gain stabilized design.

- Existing MIL-spec requirements do not provide explicit guidance in assessing HPS system design.

\section{Recommendations}

- The flexible HSV model should be further developed to include normal acceleration outputs and additional dynamic pressure cases.

- The HPS design should be further refined to define the limits of residual response reduction.

- Residual response metrics should be further developed and ultimately validated. 
This page is intentionally left blank. 


\section{REFERENCES}

1. Military Specification, "Flight Control System - Design, Installation and Test of Piloted Aircraft, General Specification for", MIL-F-9490D, 6 June 1975.

2. Military Specification, "Flight Control System, General Specification for", MIL-F-87242, 31 March 1986.

3. Military Specification, "Airplane Strength and Rigidity", MIL-A-008870A, 18 May 1972.

4. Air Force Guide Specification, "Aircraft Structures, General Specification for", AFGS-87221A, 8 June 1990.

5. Johnston, D. E., and W. A. Johnson, "Feasibility of Conventional Control Techniques for Large Highly Coupled Elastic Boost Vehicles", NASA CR-88760, March 1976.

6. Ashkenas, I. L., R. E. Magdaleno and D. T. McRuer, "Flight Control and Analysis Methods for Studying Flying and Ride Qualities of Flexible Transport Aircraft", NASA CR-172201, August 1983.

7. "The NASTRAN User's Manual (Level 17.5)", National Aeronautics and Space Administration, NASA SP-222(05), December 1978.

8. Ashley, H. and G. Zartarian, "Piston Theory - A New Aerodynamic Tool for the Aeroelasticians", J. Aero. Sc., Vol. 23, No.12, December 1956, pp. 1109-1118.

9. Pitt, D. M., "Flutter, Unsteady Aerodynamics, and Aeroservolasticity", MCAIR IRAD Project No. 7-700, 15 February 1991.

10. Myers, T. T., D. T. McRuer and D. E. Johnston, "Flying Qualities and Control System Characteristics for Superaugmented Aircraft", NASA CR-170419, December 1984.

11. Myers, T. T., D. E. Johnston and D. T. McRuer, "Space Shuttle Flying Qualities and Criteria Assessment", NASA CR-4049, March 1987.

12. McRuer, D. T., I. L. Ashkenas and D. Graham, "Aircraft Dynamics and Automatic Control", Princeton University Press, 1973.

13. Hoh, R. H., D. G. Mitchell, I. L. Ashkenas, et al, "Proposed Mil Standard and Handbook -- Flying Qualities of Air Vehicles, Vol. II, Proposed Mil Handbook", AFWAL-TR-82-3081 (II).

14. "Flying Qualities of Piloted Aircraft", MIL-STD-1797A, 30 January 1990.

15. McRuer, D. T., et al, "Flying Qualities and Control Issues/Features for Hypersonic Vehicles", NASP CR-1063, October 1989.

16. McRuer, D. T. and T. T. Myers, "Perspective on Hypersonic Vehicle Dynamic Deficiencies and Flying Qualities," Paper 467, Systems Technology, Inc., presented at 10th National Aerospace Plane Symposium, Monterey, CA, 23-26 April 1991. 
This page is intentionally left blank. 


\section{Appendix A \\ HSV PROPULSION SYSTEM INTERACTIONS WITH FLEXIBLE MODES AND FLIGHT CONTROL SYSTEMS}

The influence of the propulsion system on the longitudinal dynamics and flight control design of hypersonic vehicles (HSVs) will be reviewed here. Special emphasis is given to the interaction with the flexible mode dynamics in the context of the objectives of this contract. In several other recent and ongoing projects (References 15 and 16), STI has examined a number of dynamics, flight control and flying qualities issues for HSVs. Among the distinguishing features of HSVs compared to other aircraft are 1) very significant and complex aerodynamic/propulsion interactions, 2) significant and unusual low frequency dynamics associated with the kinematics of flight over a spherical earth and the gradient of density, thrust and other variables with altitude. There are other HSV issues, of course, including possible weathercock instabilities and problems of path/attitude consonance which will not be addressed further here.

Since the focus of this project is treatment of flexible modes in flight control design, it is to be expected that high frequency approximations are in order for the analysis. The basic high frequency approximation, see Section II-B, involves the use of the short period (constant speed) equations to represent the rigid body dynamics.

While the short period model appears to be quite adequate for the purposes of this project, lower frequency HSV dynamics will be briefly examined here because of the unusual low frequency characteristics of HSVs and, in particular, to review the influence of the propulsion system dynamics. For conventional (subsonic and supersonic) aircraft, the phugoid provides a landmark for the lower end of the vehicle dynamics (important zeros may appear below the phugoid of course). For HSVs, the "altitude" mode is generally below the phugoid. The altitude mode results from the gradients, with altitude, of several variables. The density gradient is a key effect; and thus this mode is sometimes referred to as the "density" mode. The variation of engine thrust with altitude also influences this mode. This influence is exceptional for HSVs because of their extreme range of flight conditions and the unprecedented sequence of engine configuration changes they employ; however, this particular propulsion influence appears at very low frequencies. 
The altitude mode is further complicated by interaction with kinematic "rotation of vertical" effects. These effects become significant as the aircraft reaches extreme speeds and altitudes and as aerodynamic forces diminish. These effects arise in the relation between the angular velocity (referenced to inertial space) and the Euler angles (referenced to local vertical) for flight over a spherical earth (c.f., Reference 12, pg 232). The rotation of vertical terms scale with $U / r$ and as orbital speed $U_{o r b}=\sqrt{g r}$ is approached this tends to

$$
\frac{U}{r} \approx \frac{U}{r_{e}} \rightarrow \sqrt{\frac{g_{0}}{r_{e}}} \approx 1.2 \times 10^{-3} \mathrm{rad} / \mathrm{sec}
$$

where the radius of the earth is $\mathrm{r}_{\mathrm{e}} \approx 2.08912 \times 10^{7} \mathrm{ft}$. Thus for constant attitude flight $(\Phi=$ $\Psi=0$ ), Equation 4-56 in Reference 12 shows that the steady pitch rate (with respect to inertial space) tends to

$$
\mathrm{Q}=-\mathrm{U} / \mathrm{r} \rightarrow 0.0012 \mathrm{rad} / \mathrm{sec}
$$

This corresponds to a dynamic mode, well below what we expect for conventional phugoids and, at 84 minutes per revolution, one that corresponds to the orbital (Schuler) period -- the most basic kinematic artifact for flight over a spherical earth.

For some aircraft, the crossover frequencies expected for closed loop throttle control (manual or automatic) are well below those for closed loop pitch control with elevator (or any composite pitch control from several effectors). Thus the effects of thrust loop closures can sometimes be neglected when analyzing the attitude and higher frequency dynamics; short period (constant speed) models can be then used with thrust loops neglected. This may be a valid approximation for HSVs in some cases. However, HSVs do appear to have some characteristics related to thrust control, which should be noted.

A characteristic of HSVs noted in Section II-A is a generally very low value of the $1 / \mathrm{T}_{\theta_{2}}$ zero. This is discussed in Appendix B where it can be seen that the impact is really on the path-to-attitude response and not on the attitude response per se; most notably it does not adversely effect the validity of the short period approximation for this contract.

In particular some data (Reference 16) indicate that HSVs can exhibit "backside-like" characteristics. Most notably, the $1 / \mathrm{T}_{\mathrm{h}_{1}}$ zero in the altitude to elevator transfer function can move into the right half plane. This is characteristic of conventional powered lift and 
VSTOL aircraft and can only be solved (i.e., $1 / \mathrm{T}_{\mathrm{h}_{1}}$ can only be relocated) by feedback to the throttle since no feedback to the pitch control point can modify $1 / \mathrm{T}_{\mathrm{h}_{1}}$. While perhaps unexpected for HSVs, feedbacks to the thrust control point could, in principle, be used. However, another problem unique to HSVs could cause problems here. The dynamics between thrust and throttle may have a non-minimum phase zero fundamentally associated with it due to the characteristics of some turbopump designs. This could create significant problems in designing feedback loops to the throttle. However, as noted above, all of these issues have only second order influence on the attitude and structural dynamics.

While the throttle loop dynamics should be negligible for the purposes of this project, the static effects of thrust generation on the aerodynamic stability and control derivatives have influences at much higher frequencies. The formulation in Appendix B indicates that just one stability derivative, $\mathbf{M}_{\alpha}$, and one control derivative, $\mathbf{M}_{\delta}$, are of first order importance in the superaugmented pitch loop dynamics. The longitudinal static stability enters through the approximate factor

$$
1 / T_{s p_{2}} \approx-\sqrt{\mid M_{\alpha}}
$$

and $\mathrm{M}_{\delta}$ is a factor in the loop gain. A propulsion effect is included in $\mathbf{M}_{\alpha}$ but not in $\mathbf{M}_{\delta}$ as shown in Section II.

It is certainly conceivable that there might be some propulsion system effects directly on the structural dynamics, say through aerothermoelastic effects. However, it appears that quantifying these in any generic way would be much more difficult than the already difficult issue of quantifying the aero/propulsion interactions. Thus it appears that only the static propulsion effects should be included in this work. Further data reviewed to date indicate that the power effects on static stability can be quite sensitive to configuration and this is likely true for other derivatives. Because of this configuration sensitivity and the generic nature of this project, use of very sophisticated propulsion models is not justified.

The importance of power effects is further diminished by the fact that the superaugmented pitch loop is very robust with respect to variations in the static margin (but less robust to uncertainty in control power). Beyond this, even the details of the superaugmented pitch loop, other than the $\mathrm{k} / \mathrm{s}$ asymptote above the rigid body dynamics and the loop crossover frequency, are not really significant in analyzing the flex effects. This can be seen in Figure 26 in Section III which shows a system survey of the pitch loop 
closure around the flexible aircraft using the forward gyro location (FS $=84$ ) and no structural mode filters. The Bode asymptote in Figure 26 shows a wide stretch of the desired $\mathrm{k} / \mathrm{s}$ slope around the crossover frequency $(6 \mathrm{rad} / \mathrm{sec})$. The only aerodynamic derivative directly affecting this asymptote is the control effectiveness, $\mathbf{M}_{\boldsymbol{\delta}}$. Uncertainty in this control derivative, which could arise from uncertainty in the associated power effects, would translate into effective loop gain changes that would affect the closed loop structural modes with phase stabilized modes being more sensitive to this uncertainty than gain stabilized modes. Examination of Figure 26 indicates that uncertainty in $1 / \mathrm{T}_{\mathrm{sp} 2}$ resulting from uncertainty in $\mathrm{M}_{\alpha}$ should be much less critical. Thus it can be argued that the most important power effect for this study is on the pitch control effectiveness derivative. 


\section{Appendix B}

\section{SUPERAUGMENTED PITCH LOOP DESIGN DETAILS}

This appendix presents details of the methods used to design the superaugmented pitch loop at the rigid body level.

\section{A. Formulation}

After the loop bandwidth is selected, the remaining design question is the selection of the lead $1 / T_{\mathrm{q}}$. A simple approach is given here which assumes $\tau=0$. If it is assumed for analysis that the lag-lead (Figure B-1) is scheduled perfectly, then the open loop transfer equation is that of Section III-A and is repeated here for $\tau=0$.

$$
G_{\alpha}=G_{c} G=\frac{K_{q} M_{\delta}\left(1 / T_{q}\right)}{(0)\left(1 / T_{s p_{2}}\right)}
$$

If $1 / \mathrm{T}_{\mathrm{sp} 2}$ were stable, then placing $1 / \mathrm{T}_{\mathrm{q}}$ at the same frequency but in the left half plane would produce an ideal " $\mathrm{k} / \mathrm{s}$-like" open loop transfer function with a $90 \mathrm{deg}$ phase margin at all frequencies and the loop closure would be trivial. However, since $1 / T_{\mathrm{sp} 2}$ is unstable, this strategy will produce a $\mathrm{k} / \mathrm{s}$ magnitude response, but the phase response will not be flat.

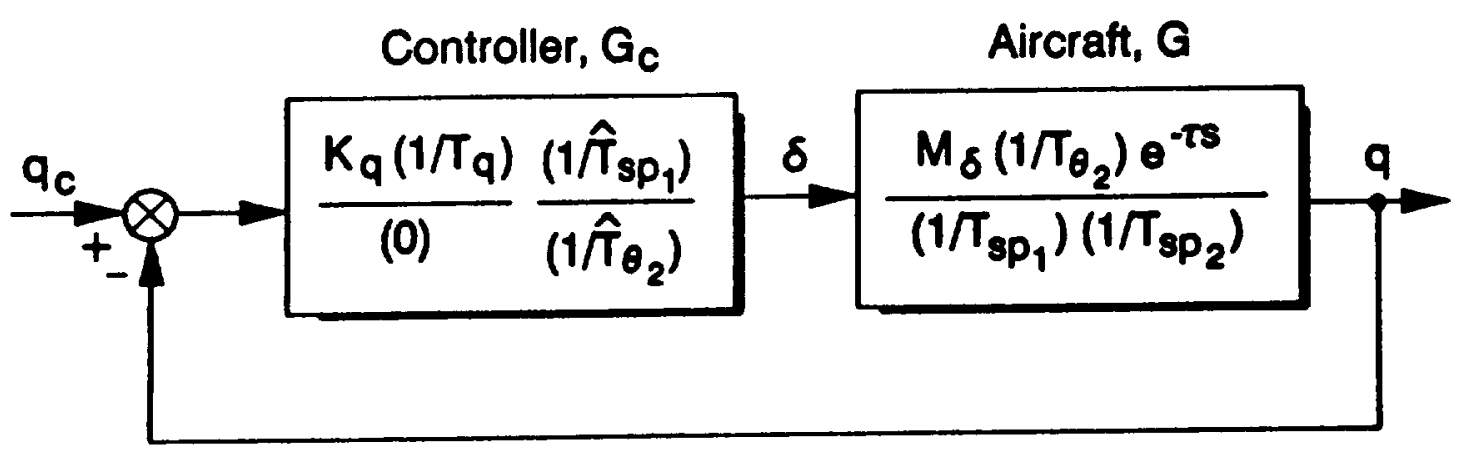

Figure B-1. Superaugmented Aircraft 
Regardless of the lead setting, the closed loop $q^{\prime} / q_{c}$ transfer function will have the form

$$
\mathrm{G}_{\mathrm{CL}}=\frac{\mathrm{q}^{\prime}}{\mathrm{q}_{\mathrm{c}}}=\frac{\mathrm{K}_{\mathrm{q}} \mathrm{M}_{\delta}\left(1 / \mathrm{T}_{\mathrm{q}}\right)}{\left[\zeta, \omega_{\mathrm{n}}\right]}
$$

The design approach proposed here assumes that the primary requirement for the pitch loop closure is to create the desired dominant closed loop mode characteristics, i.e., guarantee desired values of $\zeta$ and $\omega_{n}$. This leads to approaching the design as a pole placement problem. Because Equation B-2 has a second order denominator, simple closed form pole placement formulas for $K_{q}$ and $1 / T_{q}$ as functions of the specified $\zeta$ and $\omega_{n}$ can be developed. This development is shown in Figure B-2.

In the relationships of Figure B-2 there are six basic parameters; it is useful to distinguish three groups of these parameters:

$$
\begin{aligned}
& \text { Airframe parameters: } \mathrm{M}_{\delta} \text { and } 1 / \mathrm{T}_{\mathrm{sp} 2} \\
& \text { FCS design variables: } \mathrm{K}_{\mathrm{q}} \text { and } 1 / \mathrm{T}_{\mathrm{q}} \\
& \text { Modal specs: } \zeta \text { and } \omega_{\mathrm{n}}
\end{aligned}
$$

The final two equations in Figure B-2 are the pole placement formulas.

It is useful to normalize the FCS lead by the magnitude of the unstable short period pole -- one of the two remaining fundamental airframe parameters. From this relation Figure B-3 shows how the required lead varies with the static instability as measured by the ratio $11 / \mathrm{T}_{\mathrm{sp} 2} 1 / \omega_{\mathrm{n}}$. It can be seen that this relation is only somewhat sensitive to the specified closed loop damping ratio. In general, as the vehicle becomes more unstable, the FCS lead and the airframe pole become more separated (with the lead below the pole in frequency).

\section{B. Design Example}

The procedure will be applied to the Mach 6, power on, ascent case as an illustration. The modal parameters specified are

$$
\begin{aligned}
\zeta & =0.70 \\
\omega_{n} & =3.0 \mathrm{r} / \mathrm{s}
\end{aligned}
$$


- OPEN LOOP TRANSFER FUNCTION

$$
G_{O L}=G_{C} G=\frac{K_{q} M_{\delta}\left(s+1 / T_{q}\right)}{s\left(s+1 / T_{s p_{2}}\right)}
$$

- CLOSED LOOP CHARACTERISTIC POLYNOMIAL

$$
\begin{aligned}
\Delta^{\prime} & =1+G_{O L}=s^{2}+\left(1 / T_{s p_{2}}+K_{q} M_{\delta}\right) s+K_{q} M_{\delta} 1 / T_{q} \\
& =s^{2}+2 \zeta \omega_{n} s+\omega_{n}^{2}
\end{aligned}
$$

- dominant ClOSED LOOP MODE $\left[\zeta, \omega_{n}\right]$

$$
\begin{aligned}
\omega_{n} & =\sqrt{K_{q} M_{\delta 1} 1 / T_{q}} \\
\zeta & =\frac{1}{2}\left(1 / T_{s p_{2}}+K_{q} M_{\delta}\right) \sqrt{K_{q} M_{\delta} 1 / T_{q}}
\end{aligned}
$$

- POle Placement formulas

$$
\begin{aligned}
\frac{1}{T_{q}} & =\frac{\omega_{n}^{2}}{2 \zeta \omega_{n}+\left|1 / T_{s p_{2}}\right|} \\
K_{q} & =\frac{\omega_{n}^{2}}{M_{\delta} 1 / T_{q}}
\end{aligned}
$$

Figure B-2. Pole Placement for Superaugmented Pitch Loop 


$$
\frac{1 / T_{q}}{\left|1 / T_{s p_{2}}\right|}=\frac{1}{\left(\frac{p / T_{s p_{2}} \mid}{\omega_{n}}\right)\left(2 \zeta+\frac{\left|1 / T_{s p_{2}}\right|}{\omega_{n}}\right)}
$$

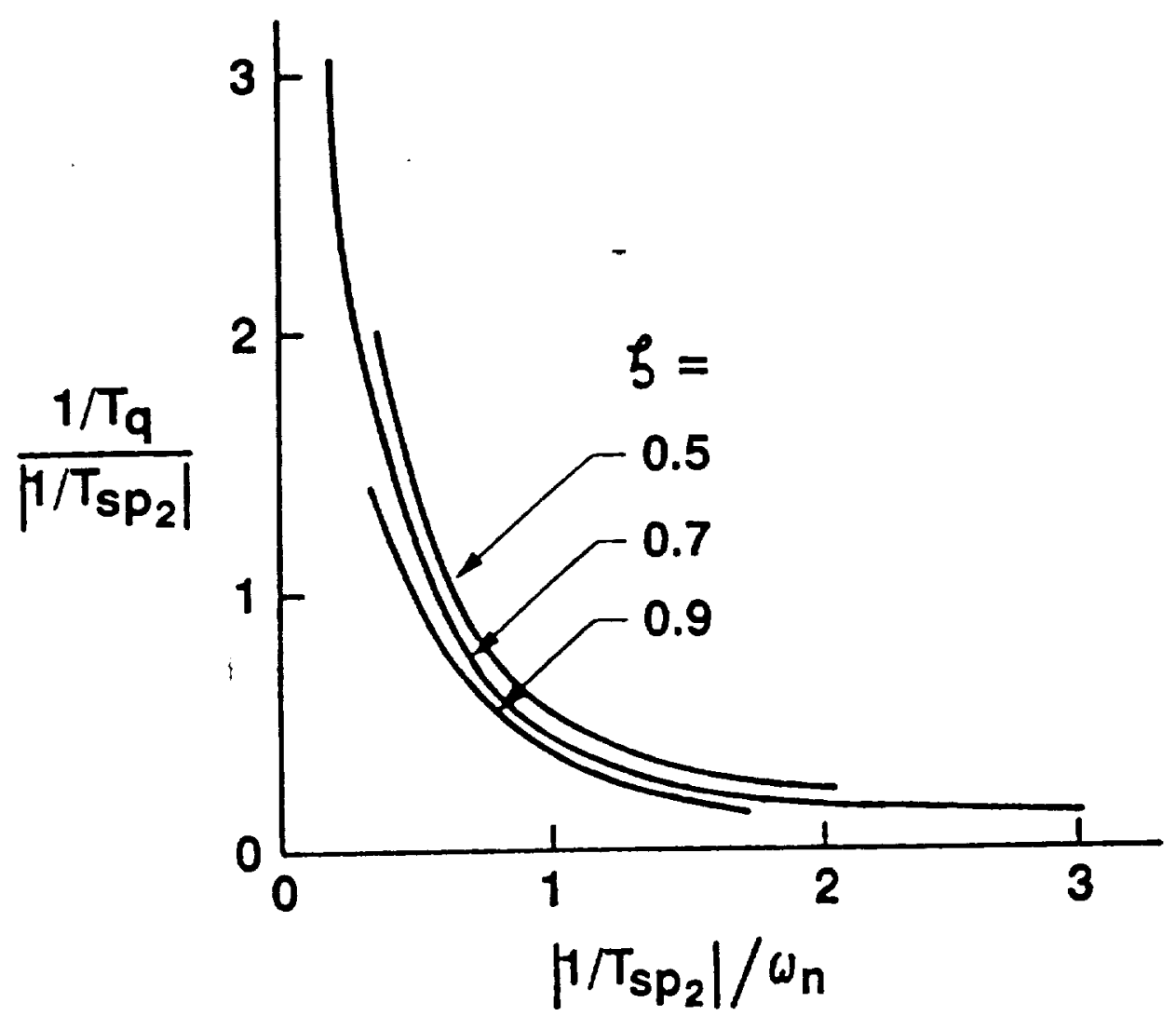

Figure B-3. FCS Lead Location as a Function of Aircraft Instability 
These relatively high values were selected to illustrate worst case difficulties.

Applying the Figure B-2 pole placement formulas gives

$$
\begin{aligned}
& \mathrm{K}_{\mathrm{q}}=1.647 \mathrm{r} / \mathrm{r} / \mathrm{s} \\
& 1 / \mathrm{T}_{\mathrm{q}}=1.451 \mathrm{r} / \mathrm{s}
\end{aligned}
$$

Figure B-4 presents a system survey of the closure. The survey verifies that the specified modal parameters are achieved. It should also be noted that the lead is well below the open loop pole in this case. The closed loop pitch rate to pitch rate command response is thus:

$$
\frac{\mathrm{q}^{\prime}}{\mathrm{q}_{\mathrm{c}}}=\frac{6.203(1.451)}{|0.70,3.00|}
$$

\section{Sensitivity of the Closed Loop Pole to Uncertainty}

The equations of Figure B-2 can be rearranged to solve for the actual values of the modal parameters as functions of the airframe parameters and the FCS variables resulting in:

$$
\begin{gathered}
\omega_{\mathrm{n}}=\sqrt{\mathrm{K}_{\mathrm{q}} \mathrm{M}_{\delta}\left(1 / \mathrm{T}_{\mathrm{q}}\right)} \\
\zeta=\frac{1}{2}\left(1 / \mathrm{T}_{\mathrm{sp}_{2}}+\mathrm{K}_{\mathrm{q}} \mathrm{M}_{\delta}\right) \sqrt{\mathrm{K}_{\mathrm{q}} \mathrm{M}_{\delta}\left(1 / \mathrm{T}_{\mathrm{q}}\right)}
\end{gathered}
$$

The two airframe parameters (and only these) should be considered uncertain. Table B-1 summarizes the partial derivatives representing the sensitivities of the closed loop mode to these open loop parameters. The expressions after the first set of equal signs for each partial are the direct derivatives of the right hand sides of Equations B-4. These expressions involve only the airframe parameters and the FCS variables, all of which are to be considered nominal values for purposes of assessing sensitivities. The nominal values of the FCS variables must be considered to covary with the nominal values of the airframe parameters due to the pole placement relations. However, the nominal values of the modal parameters are set to the modal specs and do not covary (within some reasonable design range) with the airframe parameters. Therefore, the FCS variables can be eliminated in the sensitivity partial in favor of the modal parameters using the pole placement 


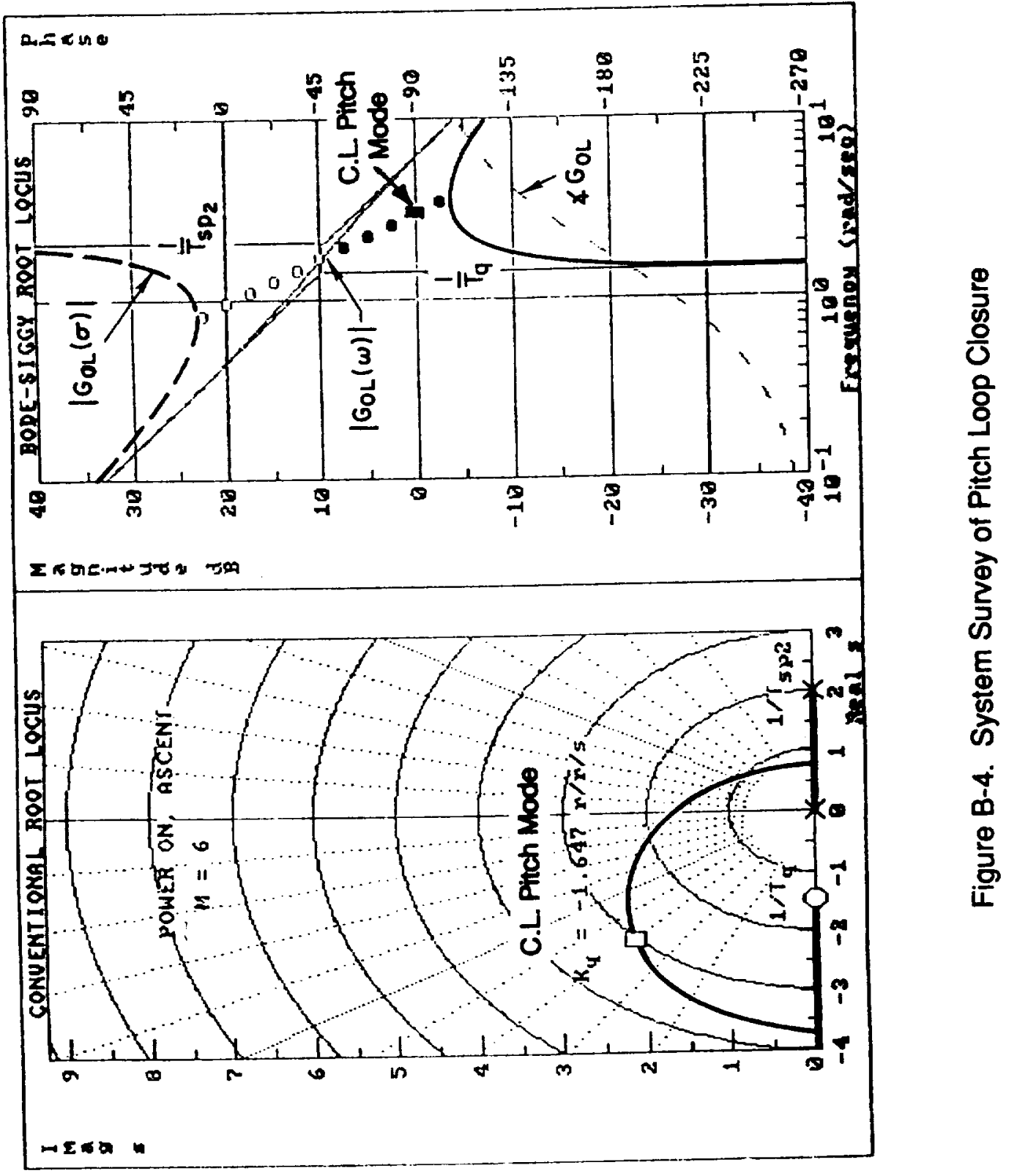



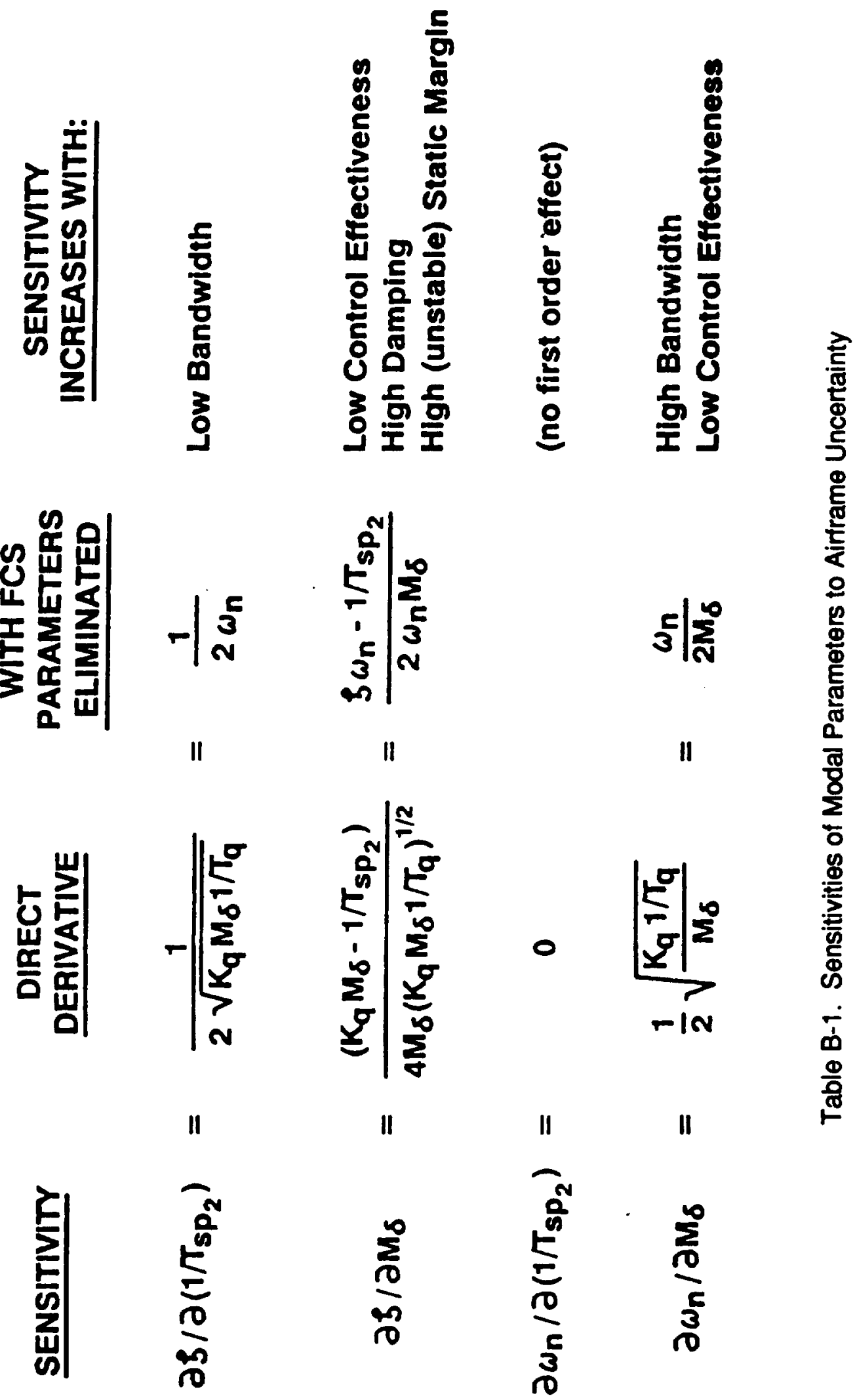
formulas. These are given as the second set of expressions in Table B-1. Since the nominal values of all of the parameters in the second set of expressions can be considered independent, these expressions can be used to consider how individual variations of nominal values affect the four sensitivities. The following observations can be made:
$\partial \zeta / \partial\left(1 / \mathrm{T}_{\mathrm{sp} 2}\right): \quad$ This sensitivity depends only on the desired bandwidth (closed loop natural frequency) and the sensitivity decreases as the bandwidth increases.
$\partial \zeta / \partial \mathrm{M}_{\delta}: \quad$ This sensitivity is increased by low values of control effectiveness. For a given damping ratio, the sensitivity increases as the relative airframe instability increases.

$\partial \omega_{n} / \partial\left(1 / T_{\mathrm{sp} 2}\right): \quad$ The actual natural frequency is not sensitive to variations in the airframe instability. It should be noted that this is true only in the sense of a first order approximation.

$\partial \omega_{n} / \partial M_{\delta}$

This sensitivity is increased by low values of control effectiveness or high values of specified modal frequency (bandwidth).

\section{Control Power Considerations}

There are obviously limits to which the pole placement procedure will give feasible designs, e.g., the gain (and hence the specified modal frequency) cannot be arbitrarily large. The primary consideration is control power. Here only the effect of control surface limiting will be considered and then only with respect to commands. Further the only control power factor that will be considered is the control surface deflection limits; rate limits will not be considered here. Another limit of particular importance here is the maximum allowable load factor because this is expected to be rather low for large hypersonic aircraft.

To begin the considerations, it is useful to examine the pitch rate, incremental load factor and control surface deflection for a $1 \mathrm{deg} / \mathrm{sec}$ pitch rate command step (Figure B-5). It is assumed here that the trim elevator deflection is zero (due say to the trimming action of a separate body flap) so that the perturbation value $\delta$ shown in Figure B-5 is the same as the total deflection and thus deflection limits can be applied directly to this variable. The load factor $n$ shown in Figure B-5 is the increment from trim which is assumed here to be 


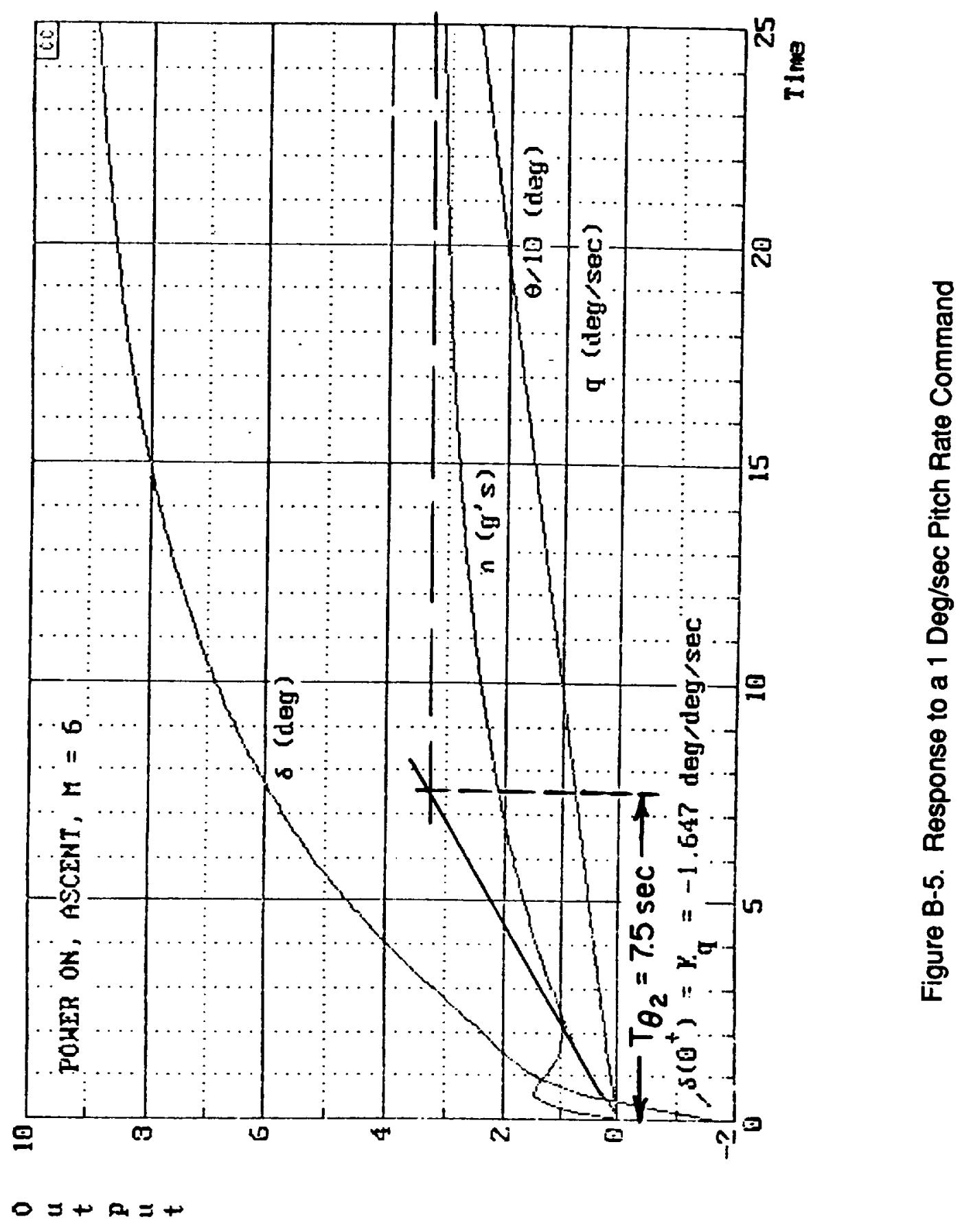


lg, although for HSV operations in general the "trim" load factor might well be less than 1; however, this is a detail which does not change the basic approach to the control power analysis. Figure B-5 shows a quite rapid rise time for the pitch rate response, which is consistent with the reasonably high dominant mode frequency. There is considerable pitch rate overshoot (about $50 \%$ ) which is due to the separation of the $1 / T_{q}$ lead from the closed loop pole (and not due to a low damping ratio which is 0.7 as specified.)

The load factor response in Figure B-5 has a much longer rise time; this is due to the very low value of $1 / \mathrm{T}_{\theta 2}$. This can be seen from the sketch in Figure B-6 which shows that an effective lag appears at $1 / \mathrm{T}_{\theta 2}$ in the load factor response to pitch rate command (effectively the pilot's stick at this level of consideration). This lag will be unconventionally low for HSVs.

Noting that the steady state value of $\mathrm{q}^{\prime} / \mathrm{q}_{\mathrm{c}}$ will be one, the steady state load factor for a step command will be

$$
\begin{gathered}
\mathrm{n}_{\mathrm{ss}}=\frac{\lim _{\mathrm{s} \rightarrow 0} \mathrm{~s} \frac{\mathrm{U}_{\mathrm{o}} / \mathrm{g}}{\left(\mathrm{T}_{\theta_{2}} \mathrm{~s}+1\right)} \frac{\mathrm{q}_{\mathrm{c}}}{\mathrm{s}}}{\mathrm{n}_{\mathrm{ss}}=\frac{\mathrm{U}_{\mathrm{o}}}{\mathrm{g}} \mathrm{q}_{\mathrm{c}}}
\end{gathered}
$$

Assuming an acoustic velocity of $1000 \mathrm{fps}, \mathrm{U}_{\mathrm{o}}$ at Mach 6 is $6000 \mathrm{fps}$, he steady state load factor for a $1 \mathrm{deg} / \mathrm{sec}(0.0175 \mathrm{rad} / \mathrm{sec})$ pitch rate computed from Equation B-5 is $3.25 \mathrm{~g}$ 's, which is consistent with Figure B-5.

The elevator response begins at a nonzero value because no actuator or other high frequency elements are included in the analysis. The initial and final values of $\delta$ are summarized in Figure B-7 and are consistent with the $\delta$ response in Figure B-5.

Since the $\mathrm{n}$ and $\delta$ responses are monotonic, their extreme values occur either at the initial or final times. Thus Equation B-5 and those in Figure B-7 cover the possible points at which elevator deflection or load factor limits can be reached and imply three distinct limits on pitch rate command. However, only the initial elevator response relationship (Figure B-7) involves an FCS parameter $\left(\mathrm{K}_{\mathrm{q}}\right)$. This leads to the question of how large $\mathrm{K}_{\mathrm{q}}$ can be before $\delta(0+)$ limits at the step pitch rate command that just produces either $n$ or $\delta$ limiting in the steady state. This question is addressed in Figure B-8 where it appears that 


$$
\begin{aligned}
\frac{n^{\prime}}{q_{c}} & =\frac{u_{0}}{g} s \frac{\gamma^{\prime}}{q_{c}}=\frac{u_{0} / g}{\left(T_{\theta_{2}} s+i ;\right.} \frac{q^{\prime}}{q_{c}} \\
& =\frac{U_{0} K_{q} M_{\delta} 1 / T_{\theta_{2}}}{g} \frac{\left(1 / \pi_{q}\right)}{\left(1 / T_{\theta_{2}}\right)\left[5, \omega_{n}\right]}
\end{aligned}
$$

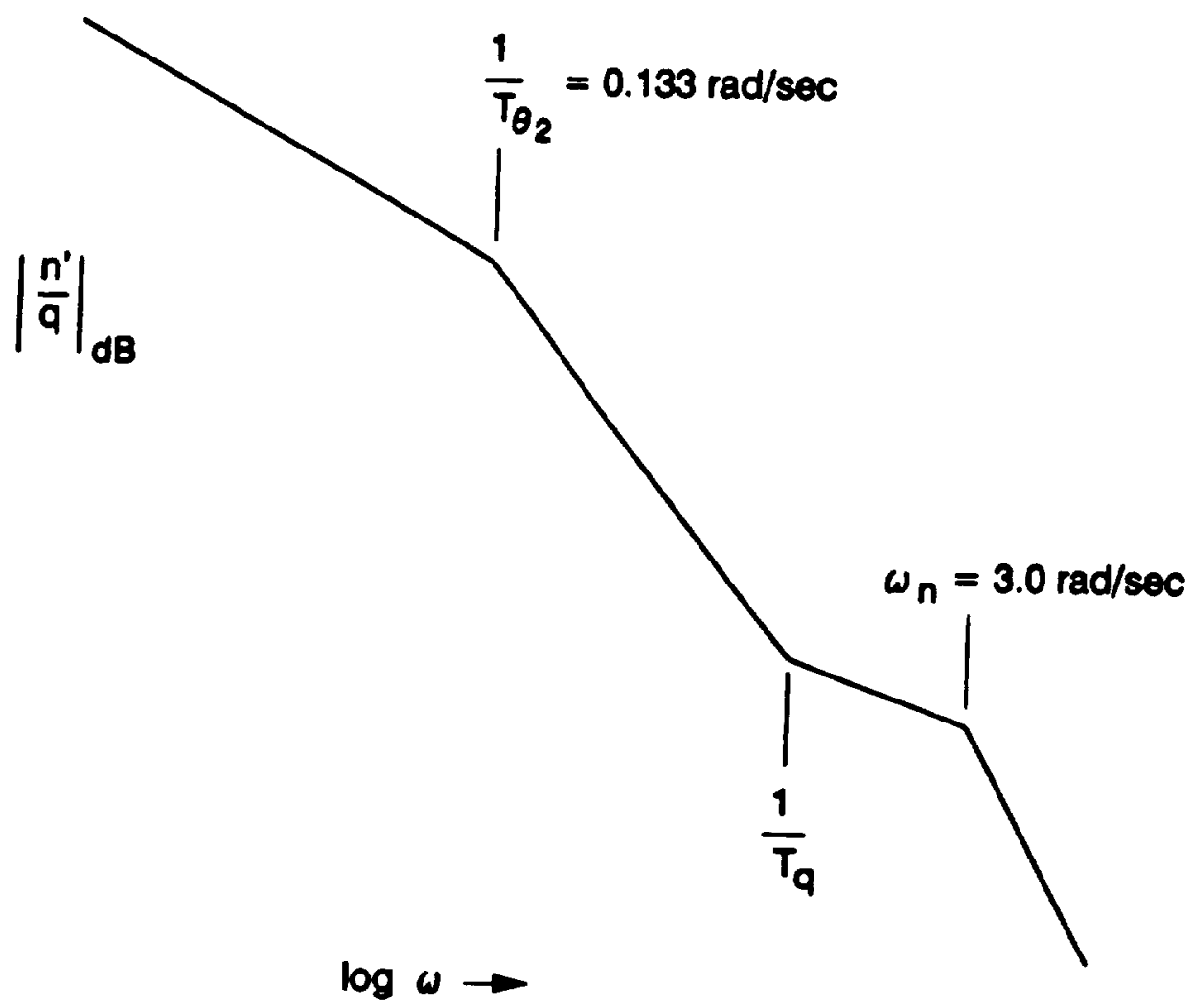

Figure B-6. Closed Loop Load Factor Response 
Initial $\delta$ response to a step $q_{c}$

Feedback is zero at $t=\sigma^{+} s o$

$$
\begin{aligned}
\delta\left(\sigma^{+}\right) & =\lim _{s \rightarrow \infty} s G_{c}(s) \frac{q_{c}}{s} \\
& =\lim _{s \rightarrow \infty} \frac{K_{q}\left(1 / \pi_{q}\right)}{(0)^{2}} \frac{\left(1 \hat{T}_{s p_{1}}\right)}{\left(1 \hat{T}_{\theta_{2}}\right)} q_{c} \\
& =K_{q} q_{c}
\end{aligned}
$$

Steady $\delta$ response to a step $q_{c}$

$$
\begin{aligned}
\frac{\delta^{\prime}}{q_{c}} & =\frac{1}{G} \frac{q^{\prime}}{q_{c}} \\
\delta_{s s} & =\lim _{s \rightarrow 0} s \frac{\delta^{\prime}}{q_{c}} \frac{q_{c}}{s} \\
& =s \lim _{s \rightarrow 0} \frac{1}{G_{s s}}\left(\frac{q}{q_{c}}\right)_{s s}^{-1} q_{c} \\
& =\frac{1 / T_{s p_{1}} * 1 / \pi_{s p_{2}}}{M_{\delta} * 1 / T_{\theta_{2}}} q_{c}
\end{aligned}
$$

Figure B-7. Initial and Final Values of Elevator Deflection 
Assumed IImits

$$
\begin{aligned}
& \delta_{\| m}=25^{\circ}=0.436 \mathrm{rad} \\
& n_{\| m}=2 \mathrm{~g}
\end{aligned}
$$

Maximum steady state pitch rate:

based on $\delta \mathrm{llmit}$

$$
q_{s s_{11 m}}=\frac{M_{\delta} * 1 / T_{\theta_{2}}}{1 / \pi_{s p_{1}} * 1 / T_{s p_{2}}} \delta_{\| l m}=2.71 \mathrm{deg} / \mathrm{sec}
$$

based on $n$ limit

$$
q_{s s_{l i m}}=\frac{g}{u_{0}} n_{l i m}=0.615 \mathrm{deg} / \mathrm{sec}
$$

$\therefore$ the load factor limit governs

Maximum galn based on $\delta\left(0^{\dagger}\right)=\delta_{\mathrm{lim}}$

$$
K_{q_{\max }}=\delta_{1 I m} / q_{s s_{l i m}}=-40.65 \mathrm{deg} / \mathrm{deg} / \mathrm{sec}
$$

Figure B-8. Maximum Loop Gain 
the critical consideration is the limit load factor. The loop gain would have to be increased by a factor of 23.5 (to $-40.6 \mathrm{rad} / \mathrm{rad} / \mathrm{sec}$ ) before the elevator would limit at the start of a maximum ( $3 \mathrm{~g}$ ) pullup. This appears to be a very large margin; however, it will be seen in the next section that other requirements, such as improved path response could, reduce the gain limit significantly.

\section{E. Shaping Response to Command with Command Path Filters}

Figure B-5 shows two undesirable features in the response to pitch rate command for the basic system: (1) excessive overshoot in the pitch rate, (2) slow load factor response. A command path filter between the pilot's stick and the $\mathrm{q}_{c}$ point can be designed to solve either, but not both, problems. For example reducing the load factor rise time will inevitably produce more pitch rate overshoot and vice versa. Furthermore, this is a fundamental physical problem which cannot be solved with any control system "trick" short of adding and using another control point, specifically a direct lift effector (which would create a whole new group of problems, of course). The reason for this can be seen in Figure B-6 where $1 / \mathrm{T}_{\theta 2}$ appears in its role as "path lag" between load factor and pitch rate. The exceptionally low values of path lag for HSVs (due to low hypersonic lift curve slopes noted previously) imply that rapid step-like changes in load factor can only be achieved by "overrotating" the pitch attitude. This can either be done by the pilot (by overrotating the stick) or a command filter can be used to accomplish the same thing for step-like stick inputs.

To examine the implications of such command filters, two special cases are developed in Figure B-9. Filter $G_{f_{1}}$ eliminates the pitch overshoot and filter $G_{f_{2}}$ reduces the lag in the load factor to a level comparable to a more conventional aircraft, i.e., an effective path lag of $0.4 \mathrm{r} / \mathrm{s}$. Figures B-10 and B-11 show the response to a step input of the pilot's stick with command filters $G_{f_{1}}$ and $G_{f_{2}}$ respectively. Figure B-10 compared to Figure B-5 shows that the $G_{f_{1}}$ filter does reduce the pitch rate overshoot essentially to zero with the expected increase in path lag which is however quite small. The initial $\delta$ is also reduced implying a larger allowable range of gain.

Figure B-11 compared to Figure B-5 shows that the $G_{\mathfrak{f}_{2}}$ filter does increase the speed of the load factor response with the expected increase in the pitch rate overshoot (to about $290 \%$ ). Further, the initial $\delta$ magnitude is increased to $-2.4 \mathrm{deg}$ which reduces the allowable gain range. 


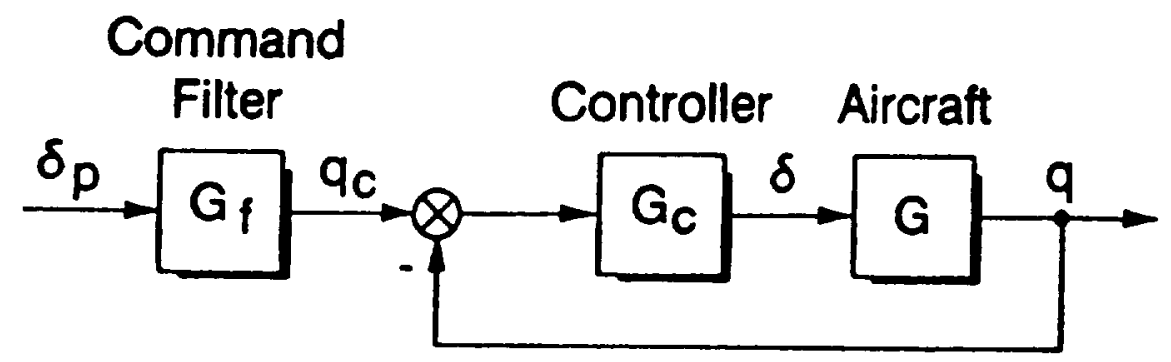

- FiLTER $G_{f_{1}}$ TO ELIMINATE PITCH RATE OVERSHOOT: CANCELS $\left(1 / T_{q}\right)$ LEAD AND REPLACES IT WITH LEAD AT $\omega_{n}$

$$
\begin{gathered}
G_{f_{1}}=\frac{\left(s / \omega_{n}+1\right)}{\left(T_{q} s+1\right)}=\frac{(0.3333 s+1)}{(0.6892 s+1)} \\
\frac{q^{\prime}}{\delta_{p}}=G_{f_{1}} \frac{q^{\prime}}{q_{c}}=\frac{K\left(\omega_{n}\right)}{\left[\zeta, \omega_{n}\right]}
\end{gathered}
$$

- filter $G_{f_{2}}$ to Reduce Path lag:

SAME AS $G_{f_{1}}$ BUT ALSO CANCELS PATH LAG

IN $n^{\prime} / q_{c}$ AND REPLACES IT WITH A LAG AT $0.4 \mathrm{r} / \mathrm{s}$

$$
\begin{gathered}
G_{f_{2}}=\frac{\left.\left(s / \omega_{n}+1\right) \hat{T}_{\theta_{2}} s+1\right)}{\left(\pi_{q} s+1\right)\left(T_{p a t h} s+1\right)}=\frac{(.333 s+1)(7.53 s+1)}{(.6892 s+1)(2.5 s+1)} \\
\frac{n^{\prime}}{\delta_{p}}=G_{f_{2}} \frac{U_{d} g}{\left(T_{\theta_{2}} s+1\right)} \frac{q^{\prime}}{q_{c}}=\frac{K_{2}\left(\omega_{n}\right)}{\left(1 / T_{\text {path }}\right)\left[\zeta, \omega_{n}\right]}
\end{gathered}
$$

Figure B-9. Command Path Filters 


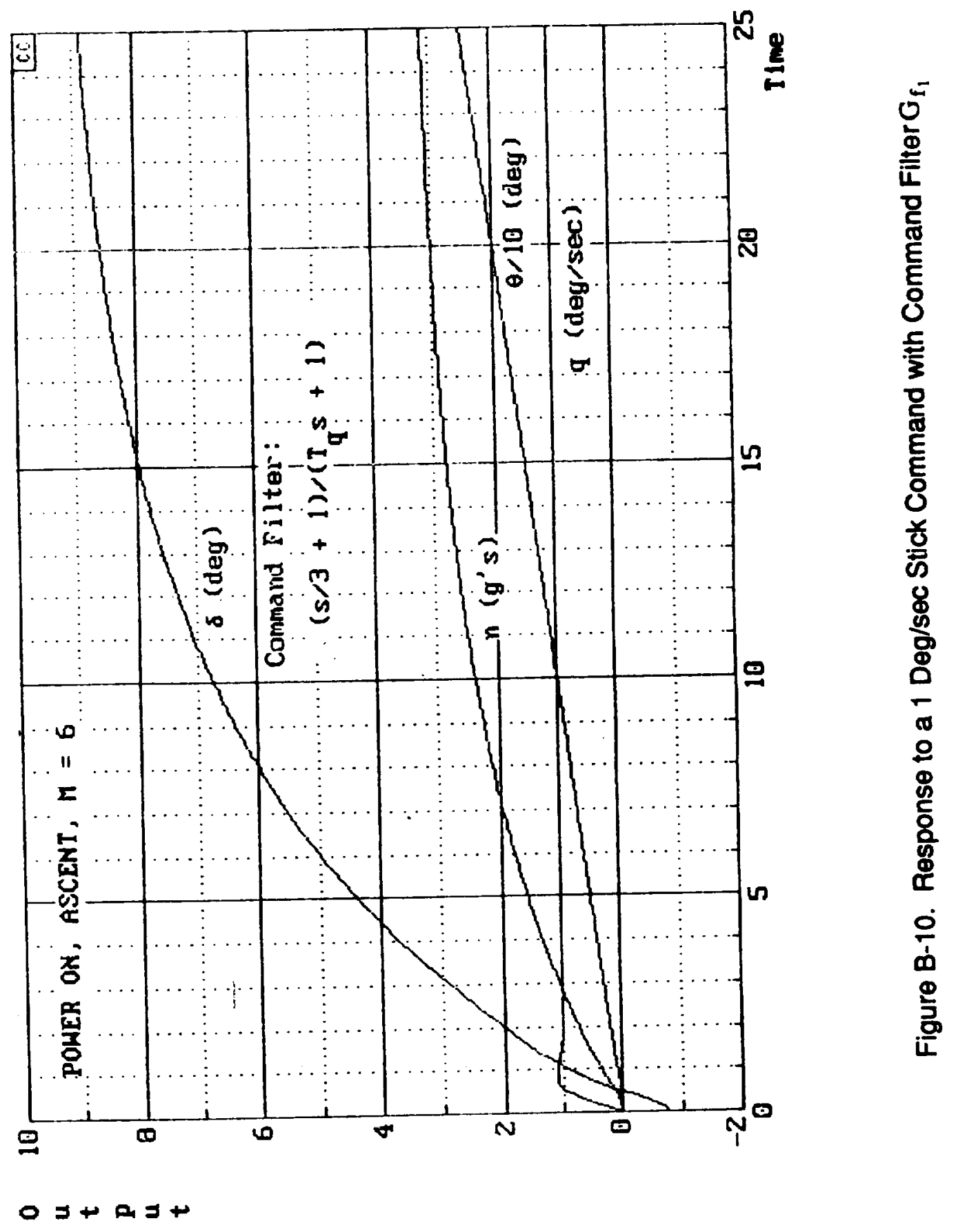




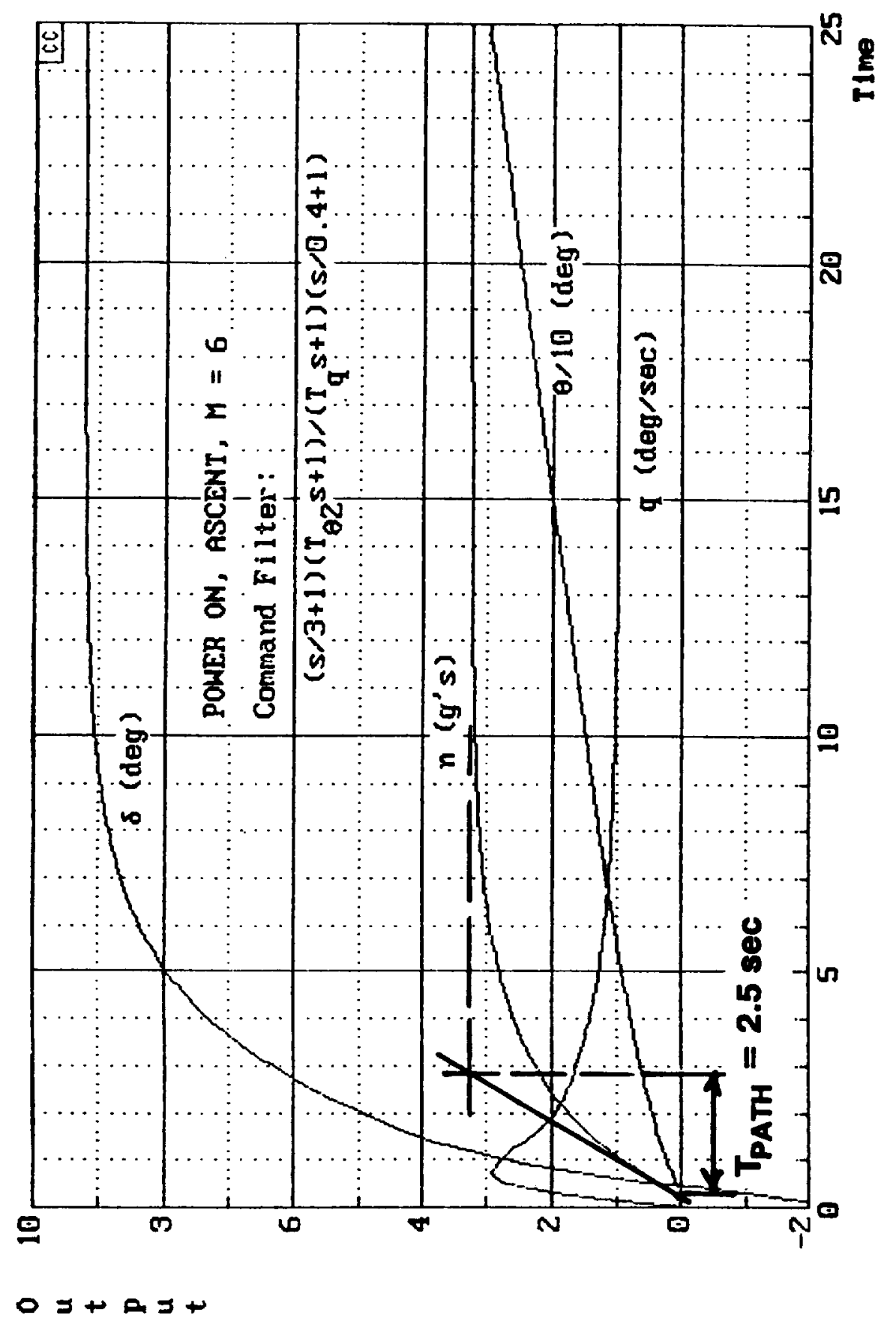

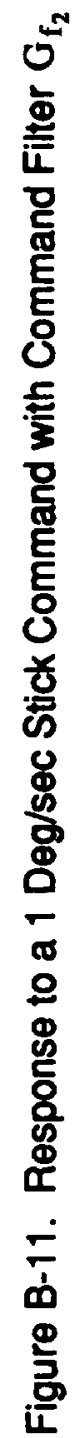




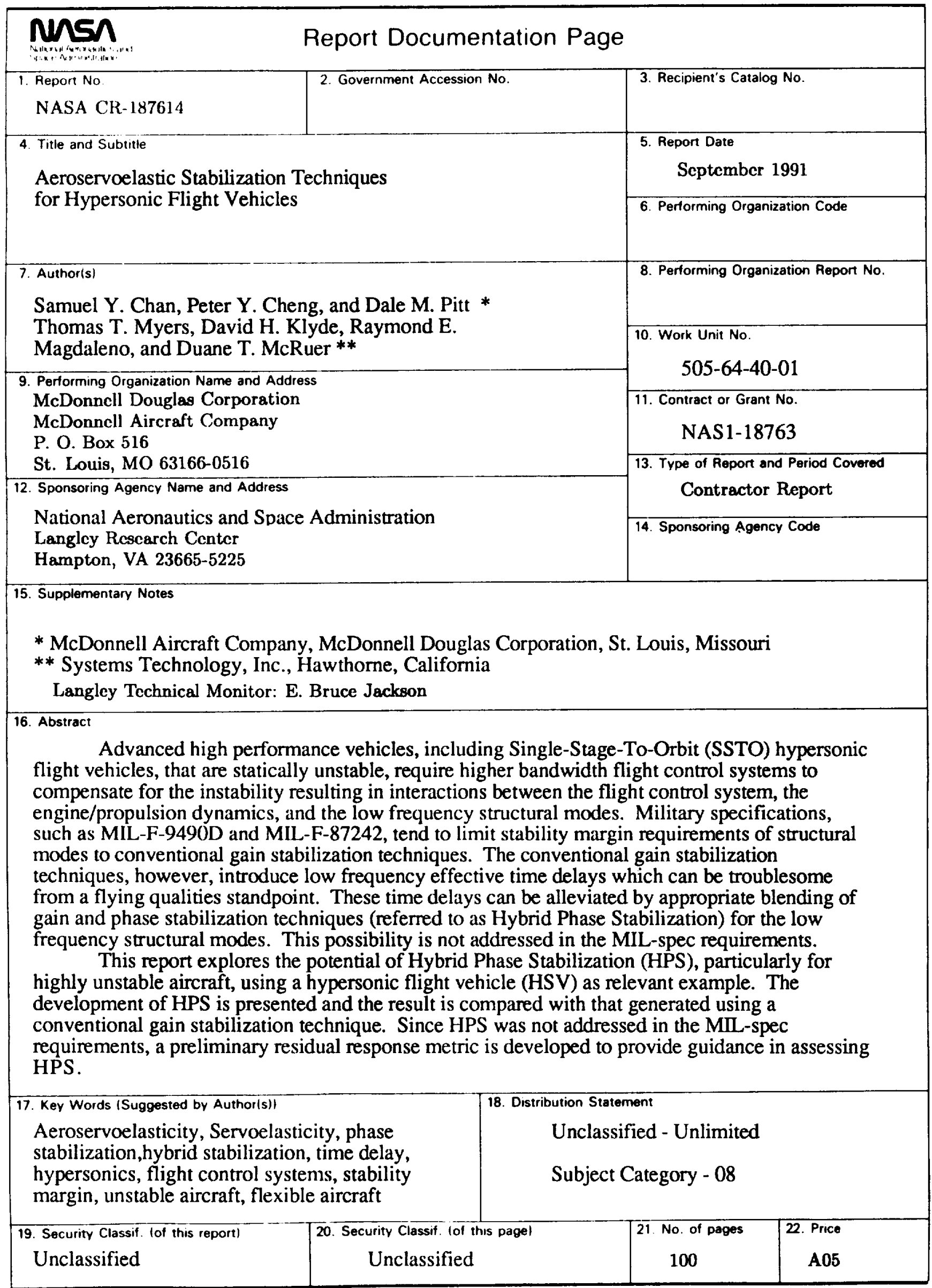

